University of Tennessee Health Science Center

UTHSC Digital Commons

$12-2007$

\title{
Theoretical and Experimental Evaluation of Spatial Resolution in a Variable Resolution X-Ray Computed Tomography Scanner
}

Roman Melnyk

University of Tennessee Health Science Center

Follow this and additional works at: https://dc.uthsc.edu/dissertations

Part of the Equipment and Supplies Commons

\section{Recommended Citation}

Melnyk, Roman , "Theoretical and Experimental Evaluation of Spatial Resolution in a Variable Resolution X-Ray Computed Tomography Scanner" (2007). Theses and Dissertations (ETD). Paper 172. http://dx.doi.org/10.21007/etd.cghs.2007.0208. 


\title{
Theoretical and Experimental Evaluation of Spatial Resolution in a Variable Resolution X-Ray Computed Tomography Scanner
}

\author{
Abstract \\ A variable resolution x-ray (VRX) computed tomography (CT) scanner can image objects of various sizes \\ with greatly improved spatial resolution. The scanner employs an angulated discrete detector and \\ achieves the resolution boost by matching the detector angulation to the scanner field of view (FOV) \\ determined by the size of an object being imaged. A comprehensive evaluation of spatial resolution in an \\ experimental version of the VRX CT scanner is presented in this dissertation. Two components of this \\ resolution were evaluated - the pre-reconstruction spatial resolution, described by the detector \\ presampling modulation transfer function (MTF), and the post-reconstruction spatial resolution, given by \\ the scanner reconstruction MTF. The detector presampling MTF was modeled by the Monte Carlo \\ simulation and measured by the moving-slit method. The modeled results showed the increase in the \\ maximum cutoff frequency (in the detector plane) from 1.53 to 53.64 cycles per $\mathrm{mm}(\mathrm{cy} / \mathrm{mm}$ ) as the \\ scanner FOV decreased from 32 to $1 \mathrm{~cm}$. The measured results supported the modeling, except for the \\ small FOVs (below $8 \mathrm{~cm}$ ), where the MTF could not be measured up to the cutoff frequency due to the \\ focal-spot limitation. The scanner reconstruction MTF was measured by the special-phantom method. \\ The measured results demonstrated the increase in the average cutoff frequency (in the object plane) \\ from 2.44 to $4.13 \mathrm{cy} / \mathrm{mm}$ as the scanner FOV decreased from 16 to $8 \mathrm{~cm}$. The MTF could not be \\ measured at the FOVs other than 8 and $16 \mathrm{~cm}$, due to the calibration-reconstruction inaccuracies and, \\ again, the focal-spot limitation. Overall, the evaluation confirmed the potential value of the VRX CT \\ scanner and produced results important for its further development.

\section{Document Type} \\ Dissertation \\ Degree Name \\ Doctor of Philosophy (PhD) \\ Program \\ Biomedical Engineering and Imaging \\ Research Advisor \\ Frank A. DiBianca, Ph.D. \\ Keywords \\ high-resolution CT, VRX CT scanner, VRX detector, spatial resolution, MTF evaluation, detector \\ presampling MTF, reconstruction MTF

\section{Subject Categories} \\ Analytical, Diagnostic and Therapeutic Techniques and Equipment | Equipment and Supplies | Medicine \\ and Health Sciences
}




\title{
THEORETICAL AND EXPERIMENTAL EVALUATION OF SPATIAL RESOLUTION IN A VARIABLE RESOLUTION X-RAY COMPUTED TOMOGRAPHY SCANNER
}

\author{
A Dissertation \\ Presented for \\ The Graduate Studies Council \\ The University of Tennessee \\ Health Science Center

\begin{abstract}
In Partial Fulfillment
Of the Requirements for the Degree

Doctor of Philosophy

In the Joint Graduate Program in Biomedical Engineering and Imaging

From The University of Tennessee

and

The University of Memphis
\end{abstract}

\author{
By \\ Roman Melnyk \\ December 2007
}


Portions of Chapters 5-7 (C) 2007 by American Association of Physicists in Medicine All other material C 2007 by Roman Melnyk 


\section{DEDICATION}

\section{To Lesya and Sophia}

For their love, support, and encouragement 


\section{ACKNOWLEDGMENTS}

I would like to thank everyone who taught me, inspired me, challenged me, supported me, and helped me throughout this process. First and foremost, I am very grateful to my advisor, Dr. Frank DiBianca, for his guidance, advice, encouragement, and understanding. My sincere thanks to Dr. Herbert Zeman and Dr. Lawrence Jordan for their valuable lessons in the area of image processing and reconstruction. I also express my gratitude to the other members of my committee, Dr. Jack Buchanan, Dr. Gary Keyes, and, Dr. Robert Ogg, for their comments and suggestions while evaluating my progress. Many thanks to Joseph Laughter for his assistance with the project hardware. I am also grateful to my colleagues at various times, Arun Gopal, Sravanthi Vangala, and David Rendon, for useful and inspiring discussions related to this study. I appreciate the help of the University of Tennessee Health Science Center Machine Shop who donated both material and labor to this project. Finally, my biggest and most heartfelt thanks go to my family - my parents, my wife, and my daughter - for their unconditional support, kind understanding, and a lot of patience.

I would like to acknowledge the American Association of Physicists in Medicine (AAPM), the Society of Photo-Optical Instrumentation Engineers (SPIE), the Institute of Electrical and Electronics Engineers (IEEE), the Institute of Physics (IOP) Publishing, and Elsevier for their permissions to use copyrighted material. I also appreciate the consents of Dr. Frank DiBianca, Dr. Jiang Hsieh, and Dr. Willi Kalender to use their published figures.

This study was partially supported by the NIH Grant No. EB-00418. 


\begin{abstract}
A variable resolution x-ray (VRX) computed tomography (CT) scanner can image objects of various sizes with greatly improved spatial resolution. The scanner employs an angulated discrete detector and achieves the resolution boost by matching the detector angulation to the scanner field of view (FOV) determined by the size of an object being imaged. A comprehensive evaluation of spatial resolution in an experimental version of the VRX CT scanner is presented in this dissertation. Two components of this resolution were evaluated - the pre-reconstruction spatial resolution, described by the detector presampling modulation transfer function (MTF), and the post-reconstruction spatial resolution, given by the scanner reconstruction MTF. The detector presampling MTF was modeled by the Monte Carlo simulation and measured by the moving-slit method. The modeled results showed the increase in the maximum cutoff frequency (in the detector plane) from 1.53 to 53.64 cycles per $\mathrm{mm}(\mathrm{cy} / \mathrm{mm})$ as the scanner FOV decreased from 32 to $1 \mathrm{~cm}$. The measured results supported the modeling, except for the small FOVs (below $8 \mathrm{~cm}$ ), where the MTF could not be measured up to the cutoff frequency due to the focalspot limitation. The scanner reconstruction MTF was measured by the special-phantom method. The measured results demonstrated the increase in the average cutoff frequency (in the object plane) from 2.44 to $4.13 \mathrm{cy} / \mathrm{mm}$ as the scanner FOV decreased from 16 to $8 \mathrm{~cm}$. The MTF could not be measured at the FOVs other than 8 and $16 \mathrm{~cm}$, due to the calibration-reconstruction inaccuracies and, again, the focal-spot limitation. Overall, the evaluation confirmed the potential value of the VRX CT scanner and produced results important for its further development.
\end{abstract}




\section{TABLE OF CONTENTS}

CHAPTER 1. INTRODUCTION

CHAPTER 2. COMPUTED TOMOGRAPHY .......................................................... 3

2.1. CT as an imaging technique ................................................................. 3

2.2. Different generations of CT scanners ................................................... 6

2.3. Reconstruction algorithms .................................................................... 11

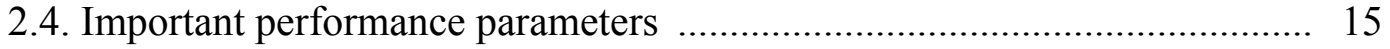

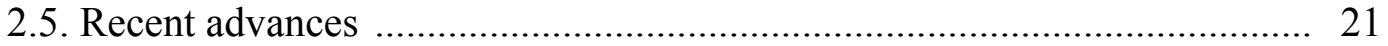

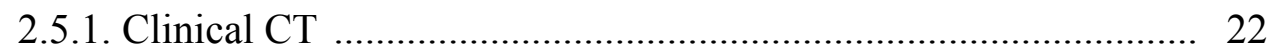

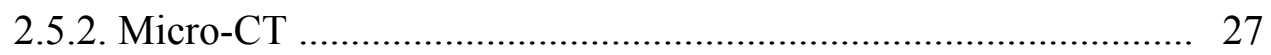

2.6. Limitations of current $\mathrm{CT}$ scanners ………………………………....... 33

\section{CHAPTER 3. VARIABLE RESOLUTION X-RAY COMPUTED}

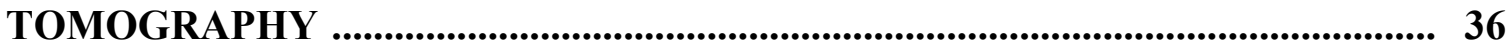

3.1. Projective compression and a VRX detector .............................................. 36

3.2. Advantages and limitations of the VRX detector ......................................... 39

3.3. Single-arm, dual-arm, and four-arm VRX detectors .................................. 44

3.4. VRX CT scanner and its potential biomedical applications ........................ 49

3.5. Development of an experimental VRX CT scanner ................................... 53

\section{CHAPTER 4. GENERAL THEORY AND METHODS FOR MEASUREMENT}

OF SPATIAL RESOLUTION .........................................................................56

4.1. Transfer-theory approach to spatial resolution ....................................... 56

4.2. Measures of spatial resolution …………………................................... 59

4.2.1. Spatial-domain measures ………………………………….... 59

4.2.1.1. Point spread function ................................................ 60

4.2.1.2. Line spread function ............................................... 62

4.2.1.3. Edge spread function ................................................ 64

4.2.2. Frequency-domain measures ...................................................... 66

4.2.2.1. Optical transfer function ............................................ 66

4.2.2.2. Modulation transfer function ..................................... 69

4.2.2.3. Phase transfer function …………………………..... 72

4.2.3. Summary of measures ............................................................. 74

4.3. Components of spatial resolution …………........................................... 76

4.4. Measurement of spatial resolution ...................................................... 84

4.4.1. Analog MTF …………………………………………..... 84

4.4.2. Detector presampling MTF ……………………………….... 89

4.4.3. Reconstruction MTF …………………………..................... 95 


\section{CHAPTER 5. METHODS FOR MODELING AND MEASUREMENT OF SPATIAL RESOLUTION IN A VARIABLE RESOLUTION X-RAY}

COMPUTED TOMOGRAPHY SCANNER ........................................................ 101

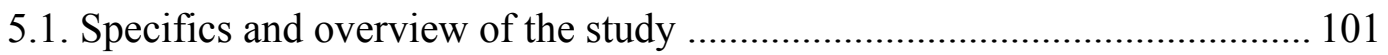

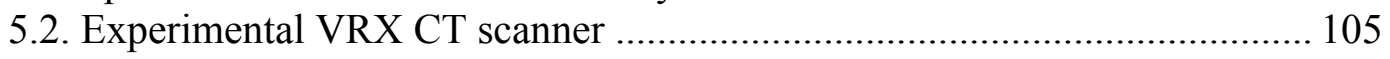

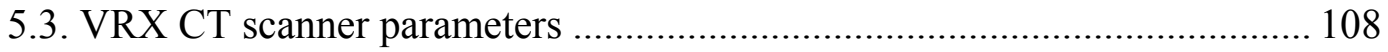

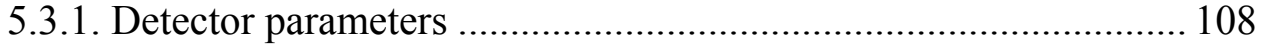

5.3.2. Scanner geometrical parameters .............................................. 110

5.3.3. X-ray beam parameters ......................................................... 114

5.4. Modeling of the detector presampling MTF ...................................... 120

5.4.1. VRX detector model ........................................................... 122

5.4.2. Monte Carlo simulation ......................................................... 124

5.5. Measurement of the detector presampling MTF .................................... 127

5.5.1. Experimental setup ........................................................ 128

5.5.2. Measurement procedure .................................................... 131

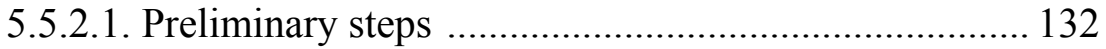

5.5.2.2. Acquisition of actual data ...................................... 134

5.5.2.3. Data processing ................................................... 135

5.5.2.4. Summary of the procedure .................................... 138

5.6. Measurement of the scanner reconstruction MTF .................................. 139

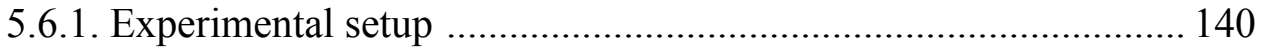

5.6.2. Measurement procedure ......................................................... 145

5.6.2.1. Preliminary steps ............................................... 145

5.6.2.2. Acquisition of actual data .................................... 147

5.6.2.3. Data processing .................................................... 149

5.6.2.4. Summary of the procedure ..................................... 151

CHAPTER 6. RESULTS AND DISCUSSION ................................................. 154

6.1. Modeling of the detector presampling MTF ....................................... 154

6.2. Measurement of the detector presampling MTF .................................. 165

6.3. Measurement of the scanner reconstruction MTF .................................. 168

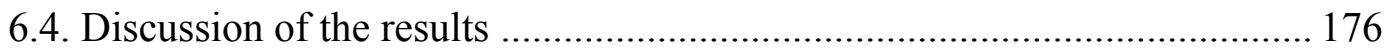

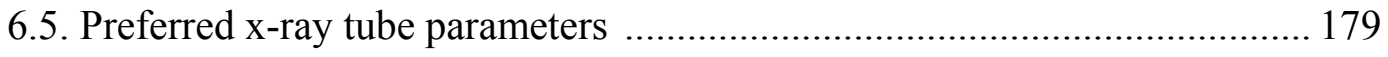

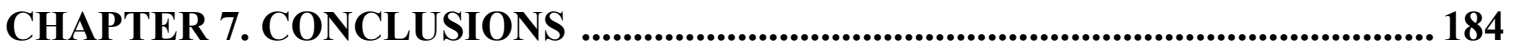

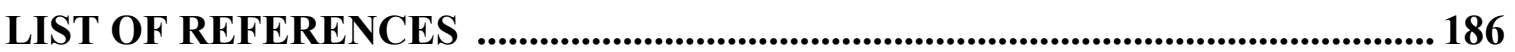

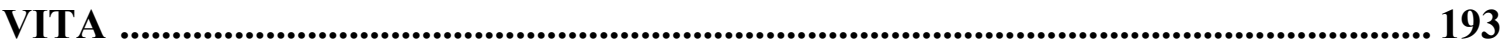




\section{LIST OF TABLES}

Table 2.1. Effective dose equivalent for standard clinical x-ray exams .................... 19

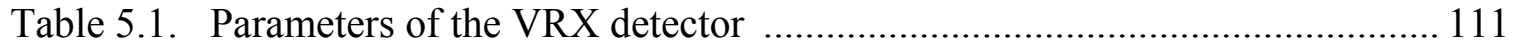

Table 5.2. Geometrical parameters of the experimental VRX CT scanner ................. 113

Table 5.3. Modeling and measurement parameters for the experimental

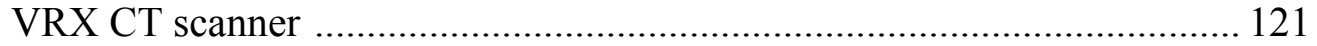

Table 5.4. Parameters of the VRX detector model ................................................ 125

Table 5.5. Parameters of the LSF phantoms for the reconstruction MTF measurement 


\section{LIST OF FIGURES}

Figure 2.1. Generations of CT scanners ............................................................... 7

Figure 2.2. Principles of backprojection …………............................................... 14

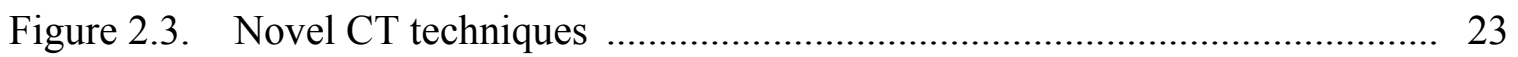

Figure 2.4. Typical micro-CT scanner ............................................................. 28

Figure 3.1. X-ray detector types .................................................................. 38

Figure 3.2. Spatial resolution of the VRX detector vs. its field of view (FOV) ........ 40

Figure 3.3. Configurations of the VRX detector ...................................................... 45

Figure 3.4. Typical VRX CT scanner ............................................................... 50

Figure 4.1. Components contributing to spatial resolution in various imaging

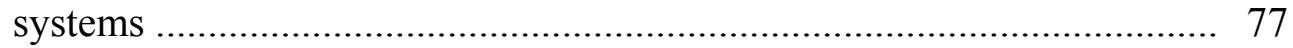

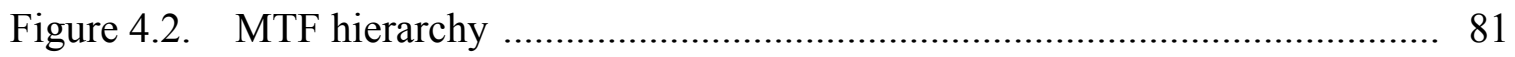

Figure 4.3. Stationary-slanted-slit method to measure the detector presampling

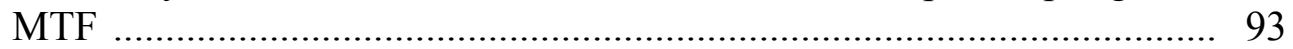

Figure 4.4. AAPM LSF phantom ................................................................... 98

Figure 5.1. Experimental VRX CT scanner ........................................................... 106

Figure 5.2. Section of a VRX detector module …………………........................... 109

Figure 5.3. Schematic diagram of the experimental VRX CT scanner ........................ 112

Figure 5.4. Theoretical phantom for computing optimum x-ray beam parameters .... 117

Figure 5.5. Optimum x-ray tube voltage for the experimental VRX CT scanner ....... 119

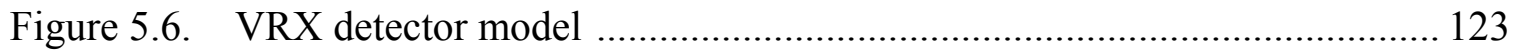

Figure 5.7. Slit and VRX detector setup for measuring the detector presampling MTF 130 
Figure 5.8. Method to determine the system magnification and the LSF sampling distance

Figure 5.9. Large, medium, and small LSF phantoms for measuring the scanner reconstruction MTF

Figure 6.1. Sample modeled and measured detector response at 8-cm FOV 155

Figure 6.2. Modeled and measured detector presampling MTF at 32-cm FOV ......... 157

Figure 6.3. Modeled and measured detector presampling MTF at 16-cm FOV ......... 158

Figure 6.4. Modeled and measured detector presampling MTF at 8-cm FOV ........... 159

Figure 6.5. Modeled and measured detector presampling MTF at 4-cm FOV ........... 160

Figure 6.6. Modeled and measured detector presampling MTF at 2-cm FOV ........... 161

Figure 6.7. Modeled and measured detector presampling MTF at 1-cm FOV ........... 162

Figure 6.8. Modeled spatial resolution of the VRX detector vs. beam quality ........... 164

Figure 6.9. Measured spatial resolution of the VRX detector vs. incident angle ....... 169

Figure 6.10. Sample measured LSF used to compute the measured scanner reconstruction MTF at 8-cm FOV 170

Figure 6.11. Measured scanner reconstruction MTF at 16-cm FOV 172

Figure 6.12. Measured scanner reconstruction MTF at 8-cm FOV 173 


\section{LIST OF ABBREVIATIONS}

\begin{tabular}{|c|c|}
\hline$\mu \mathrm{CT}$ & micro-CT \\
\hline AAPM & American Association of Physicists in Medicine \\
\hline $\mathrm{CCD}$ & charge-coupled device \\
\hline $\mathrm{CT}$ & computed tomography \\
\hline CTDI & CT dose index \\
\hline CTF & contrast transfer function \\
\hline $\mathrm{cy} / \mathrm{mm}$ & cycles per mm \\
\hline DSCT & dual-source CT \\
\hline ESF & edge spread function \\
\hline FOV & field of view \\
\hline FWHM & full width at half maximum \\
\hline FWTM & full width at tenth maximum \\
\hline $\mathrm{HU}$ & Hounsfield unit \\
\hline HVL & half-value layer \\
\hline $\mathrm{lp} / \mathrm{mm}$ & line pairs per $\mathrm{mm}$ \\
\hline LSF & line spread function \\
\hline MSAD & multiple-scan average dose \\
\hline MTF & modulation transfer function \\
\hline OTF & optical transfer function \\
\hline PSF & point spread function \\
\hline PTF & phase transfer function \\
\hline
\end{tabular}




$\begin{array}{ll}\text { SNR } & \text { signal-to-noise ratio } \\ \text { VRX } & \text { variable resolution } x \text {-ray } \\ \text { 1D } & \text { one-dimensional } \\ \text { 2D } & \text { two-dimensional } \\ \text { 3D } & \text { three-dimensional }\end{array}$




\section{CHAPTER 1. INTRODUCTION}

Computed tomography (CT) is an established method of biological x-ray imaging. It provides high-contrast two-dimensional (2D) and three-dimensional (3D) images of in vivo anatomy. Depending on the size of anatomy being imaged, CT can be divided into two general domains: clinical CT and micro-CT. Clinical CT scanners are used primarily for whole-body imaging; they have a large field of view (FOV) but only moderate spatial resolution. Although clinical CT scanners can image objects down to a few centimeters in diameter, there is no resolution improvement as the object size decreases. Micro-CT scanners, on the other hand, are designed specifically for imaging small objects. Such scanners offer high spatial resolution, but their FOV is rather limited; therefore, they cannot be used to image medium-size or large objects.

A variable resolution x-ray (VRX) CT scanner represents a novel type of a CT system that can image large as well as small objects with greatly improved spatial resolution. The scanner is based on a VRX detector - a one-dimensional (1D) discrete detector placed at an acute angle with respect to an incident X-ray beam. Because of the detector angulation, the projected size and spacing (as viewed from the x-ray source) of the detector cells are smaller than their physical size and spacing, and, hence, a resolution increase up to at least two orders of magnitude is possible. One of the advantages of the VRX CT scanner includes the ability to vary its spatial resolution according to the object size, by properly angulating the VRX detector. Due to this feature, the scanner can span both clinical CT and micro-CT domains of biological imaging and can provide the highest-possible resolution at each particular FOV. 
An experimental version of the VRX CT scanner has been built to study the scanner's performance. Among typical performance parameters, spatial resolution is of primary interest because it is the parameter the scanner promises to improve. An accurate and systematic assessment of spatial resolution is, therefore, important, in order to establish practical capabilities and limitations of the scanner, to determine its other image-quality parameters, and to optimize the scanner design.

This dissertation presents a comprehensive evaluation of spatial resolution of the experimental VRX CT scanner. Two components of this resolution were considered - the pre-reconstruction (before image reconstruction) spatial resolution and the postreconstruction (after image reconstruction) spatial resolution. Both components were evaluated in terms of the modulation transfer function (MTF). The pre-reconstruction spatial resolution was given by the detector presampling MTF, whereas the postreconstruction spatial resolution was described by the scanner reconstruction MTF. The detector presampling MTF was evaluated both theoretically and experimentally. The theoretical results showed the increase in the maximum cutoff frequency (in the detector plane) from 1.53 to 53.64 cycles per $\mathrm{mm}(\mathrm{cy} / \mathrm{mm})$ as the FOV of the scanner decreased from 32 to $1 \mathrm{~cm}$. The experimental results were in reasonable agreement with the theoretical data in all the cases where such comparison could be made. The latter results were somewhat limited by the focal-spot size. The scanner reconstruction MTF was evaluated only experimentally. The results demonstrated the increase in the average cutoff frequency (in the object plane) from 2.44 to $4.13 \mathrm{cy} / \mathrm{mm}$ as the FOV of the scanner decreased from 16 to $8 \mathrm{~cm}$. The experimental data were greatly limited by the calibrationreconstruction inaccuracies and the focal-spot size. 


\section{CHAPTER 2. COMPUTED TOMOGRAPHY}

This chapter is a brief overview of the main principles, implementations, methods, parameters, recent advances, and limitations of CT, which in the current study can be referred to as conventional CT, to differentiate it from VRX CT that will be introduced in the next chapter.

\subsection{CT as an imaging technique}

$\mathrm{CT}$ is the name given to the diagnostic imaging procedure in which anatomical information is digitally reconstructed from x-ray transmission data obtained by scanning a patient from many directions in the same plane to visualize information in that plane. ${ }^{1}$ The underlying ideas were originally developed for imaging the brain. Because the brain is encased in a very dense bony structure that absorbs most of the $\mathrm{x}$ rays, imaging the brain by radiography methods is difficult. CT was the first imaging technique to overcome this difficulty and allow slice-by-slice examination of the brain.

The mathematical principles of CT were developed by J. Radon in $1917 .^{2} \mathrm{He}$ demonstrated theoretically that an image of an unknown object could be reconstructed from an infinite set of projections through the object. Following this idea, in 1957, A. M. Cormack built the first experimental CT scanner and produced an image of a simple cylindrical phantom. ${ }^{2}$ Several years later, in 1971, G. N. Hounsfield introduced the first clinical CT scanner, specifically designed for imaging the brain. ${ }^{2,3}$ Since that time, CT has experienced tremendous technological advancement and has proven invaluable as a diagnostic tool for many clinical applications, from cancer diagnosis to 
trauma to osteoporosis screening.

The success of CT is attributed to two major advantages it offers over other available x-ray imaging techniques. First, CT provides improved contrast resolution and can distinguish between tissues of a slight attenuation difference without introducing a contrast agent. ${ }^{4}$ In fact, contrast resolution of CT is ten times better than that of screenfilm projection radiography. Such high contrast resolution results mainly from using multiple projections through a patient while avoiding attenuation outside the plane of interest; several other factors - relatively less scatter, highly efficient detectors, low detector noise, and digital manipulations of the image - contribute as well. The second advantage of CT is its ability to generate accurate tomographic images of every part of the human body without superposition of adjacent structures. ${ }^{5}$ Such superposition is the fundamental limitation in projection radiography, where the $3 \mathrm{D}$ anatomy of a patient is reduced to a $2 \mathrm{D}$ image, with no differentiation of structures along the $\mathrm{x}$-ray beam direction. ${ }^{2}$ This limitation is partly overcome in conventional x-ray tomography, where unwanted information is deliberately blurred by moving the x-ray source and film in a predetermined pattern. ${ }^{6}$ However, CT is the only x-ray imaging technique that allows complete elimination of adjacent structures and, at the same time, provides good image quality.

The tissue property that is actually computed in CT is the linear attenuation coefficient. ${ }^{7}$ For Compton scatter, which is the dominant type of x-ray interaction at the energies typically used in CT, the linear attenuation coefficient depends linearly on the electron density (i.e., tissue density and the ratio of the atomic number to the atomic mass). Most tissues in the human body (bone, fat, lung tissue, etc.) are distinguished in 
CT based on differences among their densities. Soft tissues with similar densities can be differentiated based on variations in their water content because hydrogen has a different ratio of the atomic number to the atomic mass than the other principal soft-tissue constituents.

The differentiation of tissues in $\mathrm{CT}$ is not just qualitative. Each pixel in the final image has a numerical value representing the attenuation coefficient in a small volume of the anatomy. This numerical value is a CT number, measured in Hounsfield units (HU). The relationship between CT numbers and attenuation coefficients is given by the following formula:

$$
\text { CT number }=\frac{\mu(\text { tissue })-\mu(\text { water })}{\mu(\text { water })} \times 1000,
$$

where $\mu(x)$ is the linear attenuation coefficient of the material $x$. Typical CT numbers range from about -1000 to $+3000 \mathrm{HU}$, where air has a value of $-1000 \mathrm{HU}$, soft tissues vary from -300 to $-100 \mathrm{HU}$, water is $0 \mathrm{HU}$, dense bone ranges from +1000 to $+2000 \mathrm{HU}$, and areas filled with a contrast agent have values up to $+3000 \mathrm{HU}^{2}$ Because CT numbers are quantitative, more accurate diagnosis is possible in many clinical settings.

The formation of an image in CT can be divided into four main steps. ${ }^{4}$ The first step is data collection, when transmission measurements of a collimated $\mathrm{x}$-ray beam are systematically made from many directions within a plane (or planes) of interest through a patient. For this purpose, a CT scanner includes a rotating gantry, which houses the x-ray tube and detector, and a support table, which provides a means of advancing the patient into the scanning region. The next step in the CT image formation is data pre-processing. At this step, raw data are corrected for detector non-uniformities and x-ray spectral shifts, normalized to the reference detector to correct for variations in the x-ray tube output, and 
converted to a logarithmic form. Pre-processing is done by a specialized computer. The same computer performs image reconstruction, which is the third step in the formation of a CT image. Image reconstruction involves computation of the distribution of the attenuation coefficients in the scanned area of the patient from the collected transmission data. Specific features of such computation are determined by the reconstruction algorithm. The final step of CT operation includes image display, when the result of the reconstruction is displayed on a high-quality monitor, typically in the grayscale mode, with the pixel intensities proportional to CT numbers.

\subsection{Different generations of CT scanners}

Since the first introduction of a clinical CT scanner in 1971, at least five generations of such machines have evolved. ${ }^{2}$ The main distinctions among various generations lie in the source-detector arrangement and the type of the scanning motion used to collect the data. Although earlier generations of CT scanners have now been completely replaced in the clinical environment, their discussion is important, along with current scanners, for an accurate representation of the CT evolution.

The first-generation CT scanners (Fig. 2.1-a) used a single pencil beam and a single detector in a translate-rotate scanning mode. ${ }^{1}$ The $\mathrm{x}$-ray tube and detector were first linearly translated across the FOV, acquiring 160 parallel projections. After the completion of the translation, the $\mathrm{x}$-ray tube and detector were rotated by 1 deg around a patient, and another translation was done to acquire 160 projections at this new angle. The procedure was repeated until the total rotation angle of $180 \mathrm{deg}$ was attained. Because of the serial way in which the data were collected, the first-generation scanners 


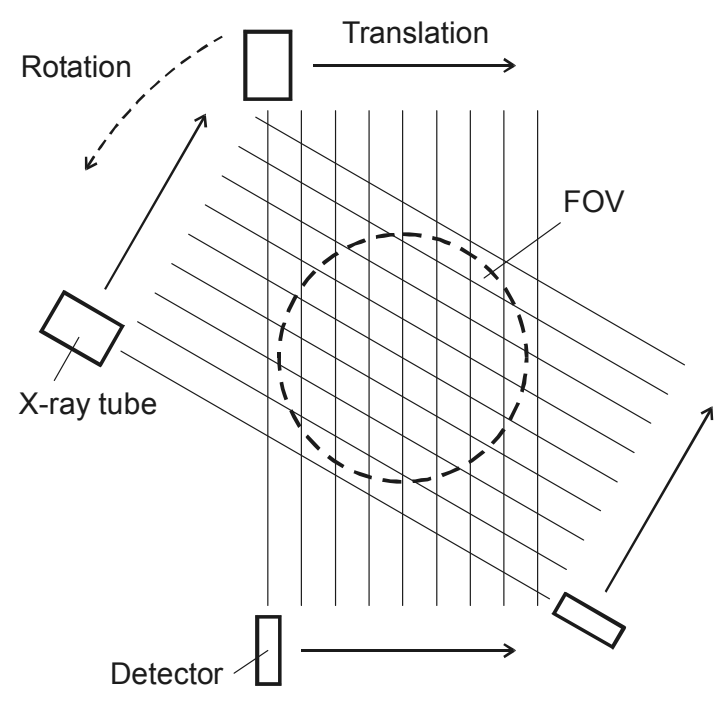

(a)

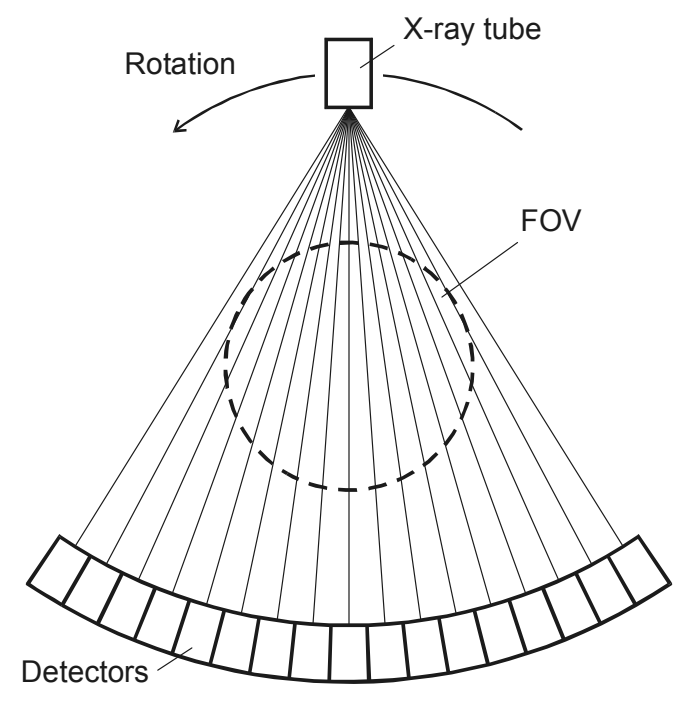

(c)

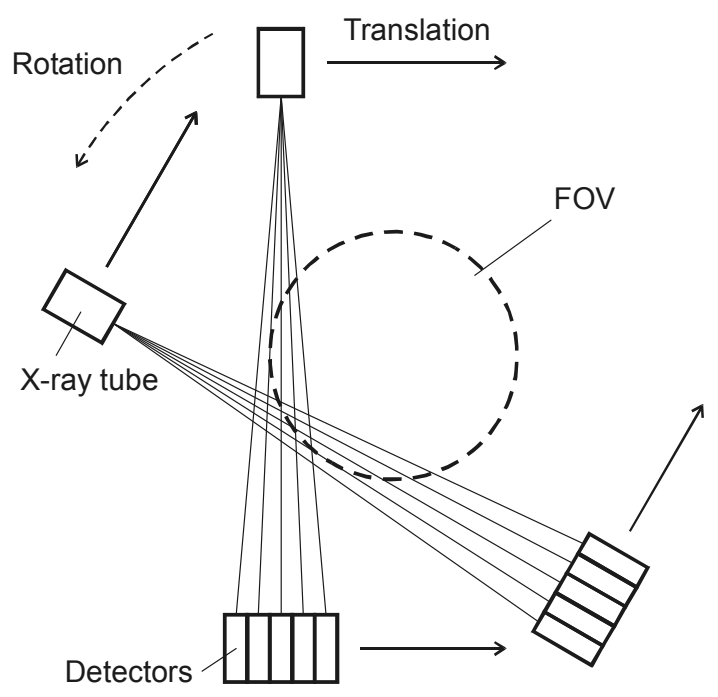

(b)

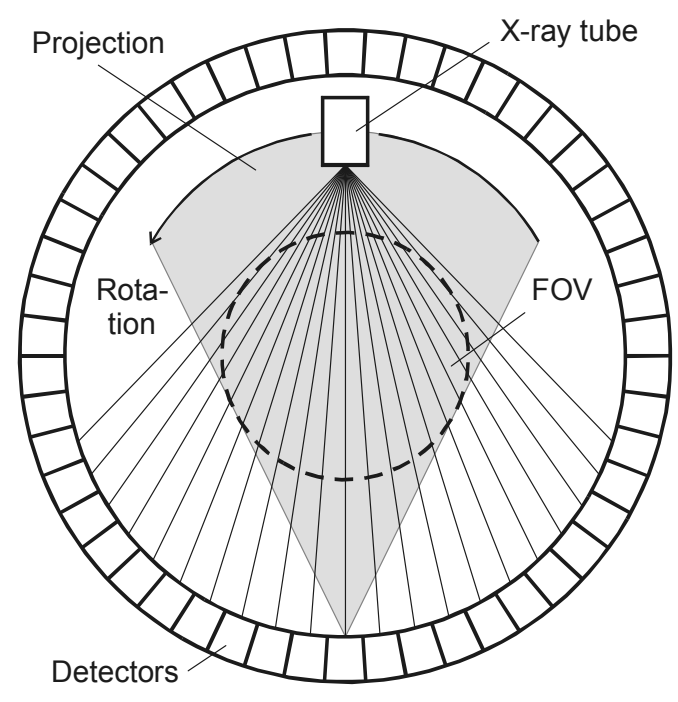

(d)

Figure 2.1. Generations of CT scanners: (a) first, (b) second, (c) third, (d) fourth, and (e) fifth. FOV is the field of view.

Source: Adapted with permission. J. Hsieh, Computed Tomography. Principles, Design, Artifacts, and Recent Advances, SPIE Press, Bellingham, 2003, pp. 12-16. 


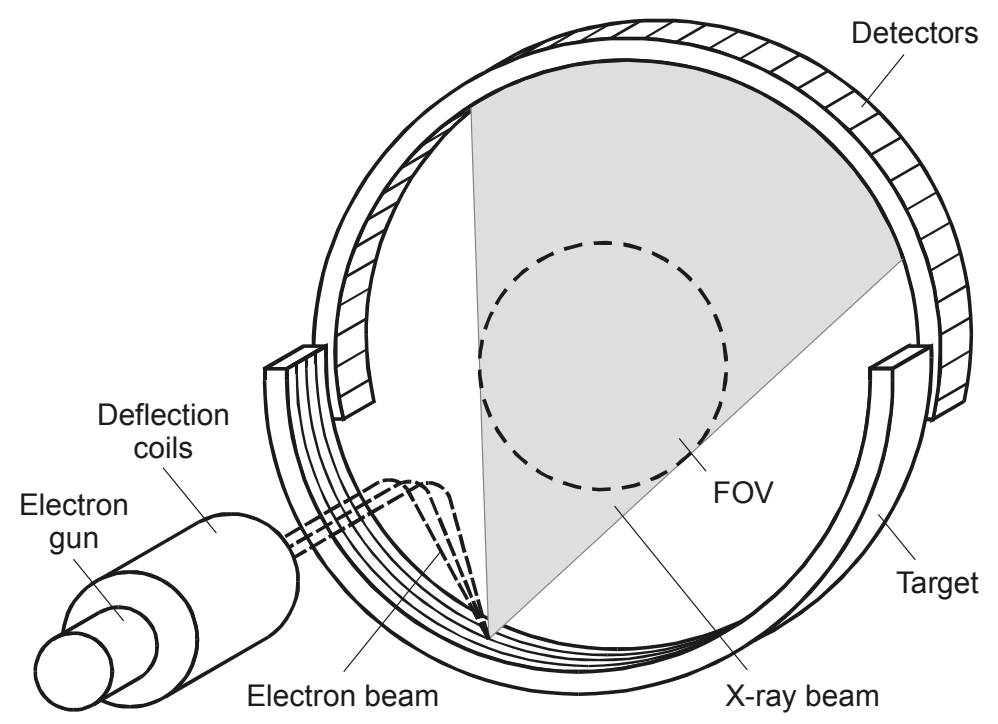

(e)

Figure 2.1 (continued). 
required at least 4.5 min to complete a scan. ${ }^{2,7}$ Such a long scan time caused serious image quality issues associated with patient motion. Therefore, the application of these machines was mostly limited to scanning the brain.

The second generation of CT scanners (Fig. 2.1-b) still operated in the translaterotate mode, but utilized a narrow fan beam (also known as multiple pencil beams) and an array of several detectors. ${ }^{6}$ Although the x-ray tube and the detector array were also rotated around a patient, the number of rotation steps was reduced approximately in proportion to the number of detectors used. With the maximum of 30 detectors in one of such scanners, the second-generation machines achieved their shortest scan time of $18 \mathrm{s.}^{2}$ This was an important milestone for body scanning because the data could be collected during the time most patients could hold their breath; hence, the majority of motioninduced artifacts could be avoided.

The most popular CT scanners are those of the third generation (Fig. 2.1-c). ${ }^{2,6,7}$ These machines use a wide fan beam and a large number of detectors (more than 800 ) located on an arc concentric to the x-ray tube. The sizes of the fan beam and the detector array are sufficiently-large to span an entire patient in one instant, thus eliminating the need for translational motion. The only motion required to collect the data in the thirdgeneration scanners is the rotation of the x-ray tube and the detector array together around the patient. Eliminating the translational motion reduced the scan time significantly. The early models could complete a scan in roughly $2-5 \mathrm{~s}$, but those models were rather inefficient because the gantry, which housed the x-ray tube and detectors, rotated both clockwise and counterclockwise to unwind the cables that transmitted the $\mathrm{x}$ ray tube power and detector signals. ${ }^{1,2}$ The acceleration and deceleration of the gantry, 
which typically weighed several hundred pounds, restricted the scan speed. Later models of the third-generation scanners employ slip rings for power and data transmission. This advancement allows the gantry to rotate at a constant speed during successive scans, reducing the scan time to $0.5 \mathrm{~s}$ or less. With such fast data acquisition, most parts of the human body can be scanned with virtually no motion-induced artifacts, and the only challenge is cardiac imaging. The introduction of the slip-ring technology was also a key to the success of helical CT, which will be discussed in a later section. Because of the inherent advantages of the third-generation scanners, nearly all of the state-of-the-art machines on the market today are of this type.

The fourth-generation CT scanners (Fig. 2.1-d) were developed to overcome the problem of ring artifacts, from which the third-generation machines suffered. ${ }^{2}$ The new scanners also use a wide fan beam and operate in the rotate-only mode. However, the detectors are removed from the rotating gantry and are placed in a stationary 360-deg ring around a patient. This arrangement requires many more detectors, up to 4800 in some recent models. While the detectors remain stationary during the scan, the x-ray tube rotates within the detector ring. As the x-ray tube moves around the patient, the data for one projection are sequentially collected by a single detector. This is different from the third-generation design, where a projection is formed by the data simultaneously collected from all the detectors. Because the same detector in a fourth-generation scanner collects all the data for one projection as well as the reference data (without patient attenuation), slight variations among the detectors do not produce ring artifacts. It should be mentioned, however, that with modern detectors and more sophisticated calibration and image-processing software, ring artifacts are essentially eliminated from the third- 
generation scanners as well. Despite their advantages, the fourth-generation machines are currently deemed impractical, due to the large number of detectors and associated electronics, especially for multislice systems.

A novel approach, representing the fifth generation of CT scanners (Fig. 2.1-e), was proposed specifically for cardiac imaging. ${ }^{2,6}$ This machine, also known as the electron-beam scanner, does not utilize any mechanical motion at all. Instead of the x-ray tube rotating around a patient, a high-energy electron beam is swept along a stationary arc-shaped anode surrounding the patient. As the electron beam is rapidly scanned along the anode, wide fan x-ray beams are generated at successive positions around the patient, thus imitating the rotation of the x-ray source. These x-ray beams are collimated to an array of detectors arranged in an arc directly opposite to the anode. The detector arc and the anode arc are offset (non-coplanar), to make room for their overlapping portions. The cathode that emits the electron beam, the coils that focus and deflect the beam, and the anode are all sealed in vacuum. Because there are no mechanical moving parts, the electron-beam scanner achieves the scan time of $50 \mathrm{~ms}$, allowing acquisition of unblurred cardiac images. When multiple target tracks are used on the anode and a multislice detector array is employed, this fifth-generation machine does fast and efficient volume acquisition and can produce fast-frame-rate $\mathrm{CT}$ movies of the beating heart.

\subsection{Reconstruction algorithms}

After x-ray transmission data acquired from many directions around a patient are pre-processed, the computer hardware and software convert these data into a CT image through a process of image reconstruction. Mathematically, reconstruction of a 2D image 
from a series of $1 \mathrm{D}$ projections requires computation of the inverse Radon transform. ${ }^{8}$ Although the most straightforward approach to computing the transform involves the direct matrix inversion, this technique is not used in practice due to its computational inefficiency and high susceptibility to noise. Therefore, a variety of alternative implementations of the inverse Radon transform have been developed for CT reconstruction. These algorithms can be divided into two general classes, the iterative and the analytic. ${ }^{1}$

The iterative (or arithmetic) reconstruction algorithms are essentially mathematical trial-and-error procedures that gradually approach the "correct" answer in a systematic fashion. ${ }^{4}$ These algorithms start with an initial guess about the $2 \mathrm{D}$ distribution of x-ray attenuation. From this guess, the predicted projection data are calculated and compared with the actual projection data acquired by the scanner. ${ }^{9}$ Discrepancies between the predicted and the measured values are used to modify the initial guess to reduce such discrepancies at the next iteration. The details of this modification depend on the specific iterative algorithm employed. The iterations are repeated until the error between the predicted projection data and the actual values becomes acceptably small.

The iterative algorithms require that all the projection data be obtained before the reconstruction can be started. ${ }^{4}$ Hence, the time of acquiring the data cannot be used for the reconstruction process. These algorithms are also computationally intensive and, therefore, inherently slower than the analytic techniques. In addition, iterative algorithms may not converge accurately due to noise in projection data. For these reasons, the iterative reconstruction algorithms, although widely utilized in the early days of CT, are no longer used in commercial scanners. These algorithms, however, are often employed 
in specialized applications, usually in the research environment, because of the improved metal artifact reduction and the ability to reconstruct images when some projections are missing. ${ }^{2,9}$

The analytic (or direct) reconstruction algorithms represent a radically different approach to $\mathrm{CT}$ reconstruction. In these algorithms, the image is reconstructed directly from the projection data, without recourse to multiple iterations and repeated comparisons between the predicted and the measured values. ${ }^{1}$ The mathematical basis for such reconstruction is given by the central-slice theorem., ${ }^{2,710}$ The most popular implementations of this theorem use the concept of backprojection (Fig. 2.2). According to this concept, the projection data acquired at each direction are projected back onto the object plane such that the value measured along a particular line is applied to all the points in the object plane that lie on that line. ${ }^{1}$ The total backprojected image is obtained by summing the contributions from all the directions. This process, known as simple backprojection, produces an image that resembles the actual object; however, the image is very blurred. ${ }^{9}$ To correct for the blurring, a filtering operation is added into the reconstruction. Although it is possible to apply a filter to the 2D backprojected image, this approach is not used in practice due to a relatively long computational time. Instead, the 1D projection data are mathematically filtered before being backprojected onto the object plane. Such filtering can be done in either the frequency domain (filtered backprojection) or the spatial domain (convolution backprojection), depending on which technique works faster for a specific filter. ${ }^{10}$ The choice of the filter is dictated by the noise level in the data, the nature of the object being reconstructed, and the ultimate diagnostic task because various filters emphasize different characteristics in the CT 


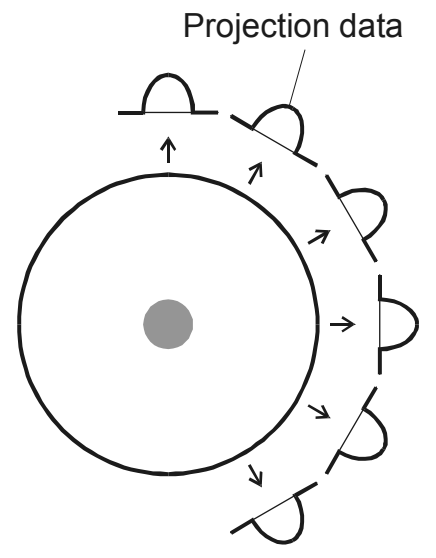

Acquisition (projection)

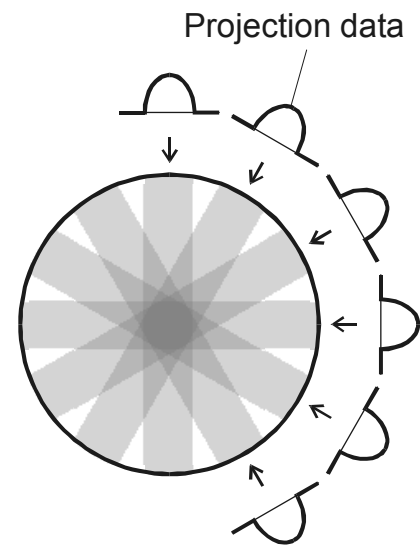

Simple backprojection
Filtered projection data

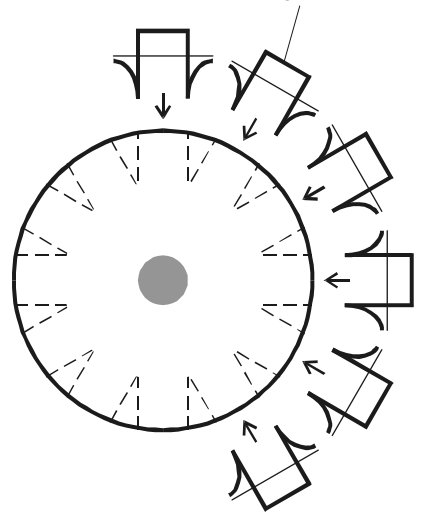

Filtered/convolution backprojection

Figure 2.2. Principles of backprojection. 
image. Most common filters include Ramachandran-Lakshminarayanan (Ram-Lak or ramp filter), Shepp-Logan, and Hamming. ${ }^{2,7,8}$

For the analytic algorithms, the reconstruction can begin as soon as the data for the first projection are acquired. ${ }^{1}$ Clearly, by allowing the reconstruction to proceed during the subsequent data acquisition, these algorithms provide a considerable saving in time. Also, because the analytic algorithms solve the reconstruction problem in one pass through the data, they are inherently faster than the iterative methods. ${ }^{4}$ The analytic algorithms are less susceptible to noise, as well, and permit task-based adjustment of the image quality (by selecting a proper filter). Due to these practical advantages, the analytic reconstruction algorithms, specifically in the form of filtered/convolution backprojection, are most frequently used in commercial CT scanners today. One of the limitations of these algorithms is that a complete set of projection data must be available in order for the reconstruction to work.

\subsection{Important performance parameters}

The performance of a CT scanner as an imaging device can be characterized by such key parameters as spatial resolution, contrast resolution, noise properties, and radiation dose. In addition, due to the specifics of this imaging modality and its recent developments, several other factors including CT-number accuracy, image artifacts, and temporal resolution are also important for the performance evaluation.

Spatial resolution of a CT scanner describes the scanner's ability to resolve

closely placed objects. ${ }^{2}$ Spatial resolution is measured in two orthogonal directions: inplane $(x-y)$ and cross-plane $(z)$. The in-plane spatial resolution is typically specified in 
line pairs per $\mathrm{mm}(\mathrm{lp} / \mathrm{mm})$ or cycles per $\mathrm{mm}(\mathrm{cy} / \mathrm{mm})$. Compared with screen-film projection radiography, CT has much lower in-plane resolution (only $0.5-2 \mathrm{lp} / \mathrm{mm}$ vs. 4$20 \mathrm{lp} / \mathrm{mm}$ for the radiography). Also, this resolution in CT is generally not isotropic but depends on the direction and location in the image. As a result, the in-plane spatial resolution is often separated into a radial component (the resolution along a line from the center of the image to its periphery) and an azimuthal, or circumferential, component (the resolution along a concentric circle in the image). ${ }^{9}$ Both components are influenced by the x-ray tube focal-spot size, detector aperture, system magnification, reconstruction algorithm, pixel matrix, and patient motion. ${ }^{2,69}$ In addition, the radial resolution is greatly affected by the number of rays in a CT scan. The azimuthal resolution, on the other hand, is largely determined by the number of projections through the object. Compared with the in-plane resolution, the cross-plane (or axial) spatial resolution lags notably behind, but this difference is quickly disappearing with recent $\mathrm{CT}$ advances. ${ }^{2}$ The cross-plane resolution is usually specified by the full width at half maximum (FWHM) and the full width at tenth maximum (FWTM) of the slice sensitivity profile. This resolution mainly depends on the x-ray collimation in the $z$ direction, detector thickness, and system magnification. A detailed description of CT spatial resolution, both the in-plane and the cross-plane, will be given in Chapter 4.

Contrast resolution refers to the ability of a $\mathrm{CT}$ scanner to differentiate a lowcontrast object from its background. ${ }^{4}$ Quantitatively, object contrast is defined as the percentage difference in the linear attenuation coefficients between the object and the background. The smallest percentage difference that can be detected gives a value of the contrast resolution. Thus, a scanner with $1 \%$ contrast resolution would be able to 
distinguish an object whose mean $\mathrm{CT}$ number differs from the background by $10 \mathrm{HU}{ }^{2}$ CT, by far, has the best contrast resolution of any clinical x-ray modality. Whereas the minimum detectable contrast for screen-film projection radiography is approximately $5 \%$, CT demonstrates contrast resolution of about $0.5 \%$, ten times better. ${ }^{9}$ Unlike spatial resolution, contrast resolution has no rigorous mathematical definition. The difficulty with such a definition arises from the fact that the contrast resolution depends not only on the contrast of an object but also on its size and the noise level. ${ }^{2}$ In addition, the evaluation of the contrast resolution is highly dependent on an individual human observer. Because there is no exact method to asses contrast resolution, a number of statistical approaches aimed at specific diagnostic tasks are used instead. The design parameters that influence contrast resolution include the x-ray tube voltage and current, slice thickness, and reconstruction algorithm.

The level of noise in the image is another important factor of the CT scanner's performance. A typical measure of noise consists of the standard deviation of the pixel values in a reconstructed image of a uniform object (e.g., water). There are, in general, three major sources of noise in $\mathrm{CT} .^{2}$ The first source includes the quantum noise determined by the number of x-ray photons being detected. In a properly-operating CT scanner, the quantum noise must dominate. ${ }^{4}$ This type of noise is mainly influenced by the scanning technique (x-ray tube voltage and current, slice thickness, scan speed, etc.) and the detector efficiency. The second source of noise refers to the inherent physical limitations of the CT scanner. Such limitations include optical and electronic noise in the detector, electronic noise in the data acquisition system, scattered radiation, and many other factors. The third source is the noise associated with the image-generation process. 
This type can be further divided into the reconstruction noise and the calibration noise. The former depends on the reconstruction filter, pixel matrix, and any post-processing techniques, whereas the latter is affected by the errors in the scanner calibration.

Radiation dose of a CT scan indicates its risk to the patient's health. Radiation dose is usually expressed as absorbed dose, which corresponds to energy deposited per unit mass of tissue. ${ }^{2,9}$ The absorbed dose is measured in grays (Gy) or rads $(1 \mathrm{rad}=$ $0.01 \mathrm{~Gy})$. CT has much higher radiation dose than projection radiography. Also, the dose distribution is different in CT. The main differences include a substantially smaller volume of the irradiated tissue and a more uniform energy deposition. Due to these differences, CT-specific measures of radiation dose are often employed, such as the integral dose, ${ }^{9} \mathrm{CT}$ dose index (CTDI), ${ }^{8}$ and multiple-scan average dose (MSAD) ${ }^{2}$ In addition, the effective dose equivalent, measured in sieverts (Sv) or rems $(1 \mathrm{rem}=$ $0.01 \mathrm{~Sv})$, is frequently used to provide an overall indication of the patient risk. ${ }^{8}$ Typical values of the effective dose equivalent for $\mathrm{CT}$ and other standard clinical $\mathrm{x}$-ray exams are listed in Table 2.1. The legal limit in annual radiation dose in the US is $0.05 \mathrm{~Sv}$, which corresponds to 15 head CT scans or 5 full-body scans. For a particular CT scanner, radiation dose depends on the $\mathrm{x}$-ray tube voltage and current, $\mathrm{x}$-ray beam quality, slice thickness, and scan time.

The performance parameters described so far - spatial resolution, contrast resolution, noise, and radiation dose - are not independent but affect CT image quality in a related manner. In fact, there exists a well-established relationship ${ }^{4}$ among these parameters: 
Table 2.1. Effective dose equivalent for standard clinical x-ray exams.

\begin{tabular}{lc}
\hline \multicolumn{1}{c}{ Clinical exam } & $\begin{array}{c}\text { Effective dose } \\
\text { equivalent }(\mathrm{mSv})\end{array}$ \\
\hline Breast (mammography) & 0.05 \\
Chest (radiography) & 0.03 \\
Skull (radiography) & 0.15 \\
Abdomen (radiography) & 1.0 \\
Barium fluoroscopy & 5 \\
Head CT & 3 \\
Body CT & 10 \\
\hline
\end{tabular}

Source: Adapted with permission. A. Webb, Introduction to Biomedical Imaging, IEEE Press, Piscataway, 2003, p. 48. (C) 2003 IEEE. 


$$
\sigma^{2} \propto \frac{1}{r^{3} h D}
$$

where $\sigma$ is the standard deviation of the pixel values in a uniform image, $r$ is the spatialresolution element size, $h$ is the slice thickness, and $D$ is the absorbed dose. According to this relationship, a twofold improvement in spatial resolution, with the other parameters being the same, would require an eightfold increase in radiation dose. Similarly, an improvement in contrast resolution corresponding to a twofold reduction in noise would involve a quadrupling of radiation dose. This result is a consequence of the statistical properties of the x-ray beam, and there appears to be no way to significantly improve such a trade-off. ${ }^{7}$

CT-number accuracy plays an important role in the scanner's performance because radiologists often rely on values of the measured CT numbers for diagnosis. There are two aspects of CT-number accuracy: consistency and uniformity. ${ }^{2} \mathrm{CT}$-number consistency dictates that for the same phantom scanned with different slice thicknesses, at different times, or in the presence of other objects, CT numbers should be the same. CTnumber uniformity demands that the values should not change with the pixel location in a uniform phantom, or with the phantom position in the FOV. Both CT-number consistency and uniformity can only be maintained within a reasonable range, due to numerous types of artifacts occurring in the image. ${ }^{9}$ Moreover, CT-number accuracy can be significantly affected by the reconstruction algorithm.

Image artifacts are clinically-relevant discrepancies between a reconstructed image and the actual object. Such discrepancies are inevitable in any CT scanner and degrade its performance. Image artifacts can be classified according to their origin. ${ }^{5}$ The physics-related artifacts include beam hardening, partial volume effect, and scattered 
radiation. The patient-related errors involve patient (or organ) motion and presence of foreign metal objects. The scanner-related effects embrace insufficient detector calibration, drifts (time-, temperature-, or irradiation-history-dependent) in the detector response, deficiencies in the reconstruction algorithm, non-uniform scanning motion, and fluctuations in the x-ray tube voltage. All the above errors show up in the reconstructed images with different typical patterns, which further vary with the scanner generation. Some image artifacts can be corrected. Others cannot; they present an annoyance to radiologists and may cause misdiagnosis. ${ }^{2}$

Temporal resolution is becoming an increasingly important performance factor in CT due to recent application of this modality to fluoroscopy and cardiac imaging. ${ }^{2}$ These two areas, however, employ different aspects of temporal resolution. CT fluoroscopy, most commonly used for needle guidance during biopsies, stresses the real-time nature of generated images. The main time-related parameter for CT fluoroscopy is, therefore, the scanner's temporal response, determined by the delay between an actual movement of a needle and a display of this movement in the image. CT cardiac imaging, on the other hand, emphasizes the ability to "freeze" cardiac motion. Hence, the key descriptor of the scanner's performance in the time domain for cardiac imaging is the temporal span of a reconstructed image relative to the cardiac motion cycle. This temporal span depends primarily on the scan speed of a CT scanner.

\subsection{Recent advances}

In recent years, due to continuous improvements in the acquisition geometry, $\mathrm{x}$ ray tube design, detector technology, and computer speed, CT has undergone several 
major developments. A large fraction of these developments has been focused on enhancing the performance of CT scanners designed for imaging the human body. At the same time, CT has been applied to scanning non-human subjects such as small animals or specimens, and substantial progress has been made in this area as well. Because of difference in the size of anatomy being imaged in each case, CT can be divided into two general domains: clinical CT and micro-CT. Each of these domains has been following its own path of development.

\subsubsection{Clinical CT}

Clinical CT deals with imaging of the human body. Recent advances in clinical CT include techniques that have already been established commercially, such as helical and multislice CT, as well as methods that are just entering the market, such as conebeam and dual-source CT.

Helical CT (also inaccurately called spiral CT) was introduced in the early 1990s with the purpose of reducing the scan time of the existing, step-and-shoot scanners. ${ }^{2,8}$ In helical CT, data are acquired while a patient is slowly translated through the scanner's gantry (Fig. 2.3-a). The resulting helical movement of the x-ray tube around the patient

allows rapid volumetric data acquisition over large areas of the body. In some instances, the entire scan can be performed within a single breath-hold of the patient, so that artifacts resulting from respiratory motion can be avoided. ${ }^{9}$ To achieve a short scan time ( $0.5 \mathrm{~s}$ or less per rotation), helical CT relies on the slip-ring technology of the third- and fourth-generation machines. One of the main parameters of a helical CT scanner is its pitch. The pitch describes the number of slice thicknesses the patient moves during one 


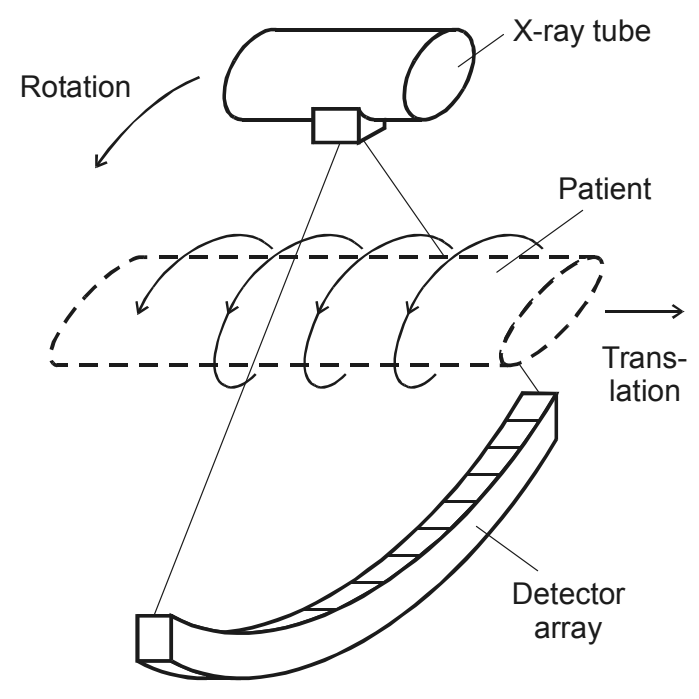

(a)

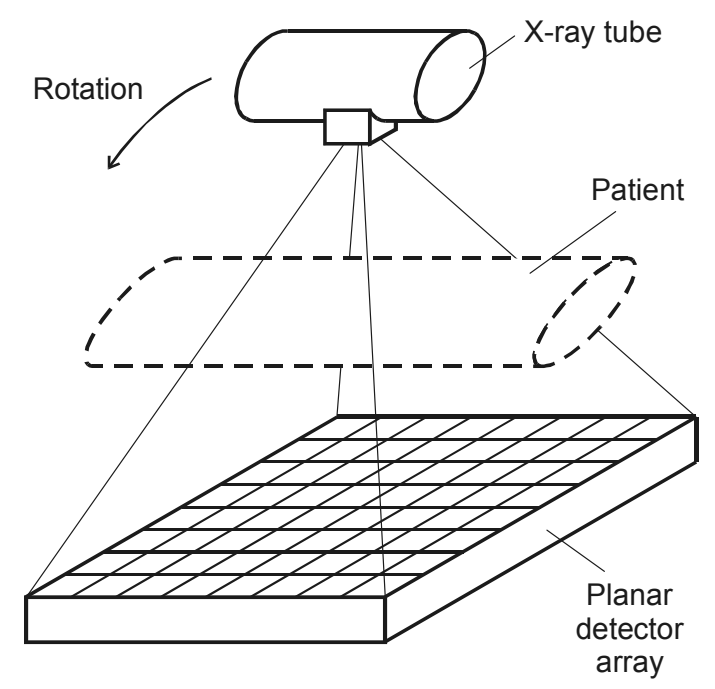

(c)

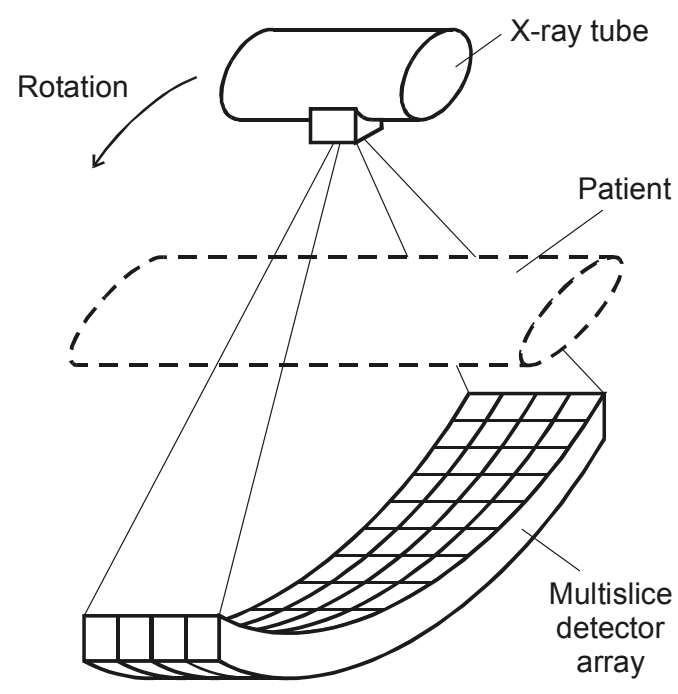

(b)

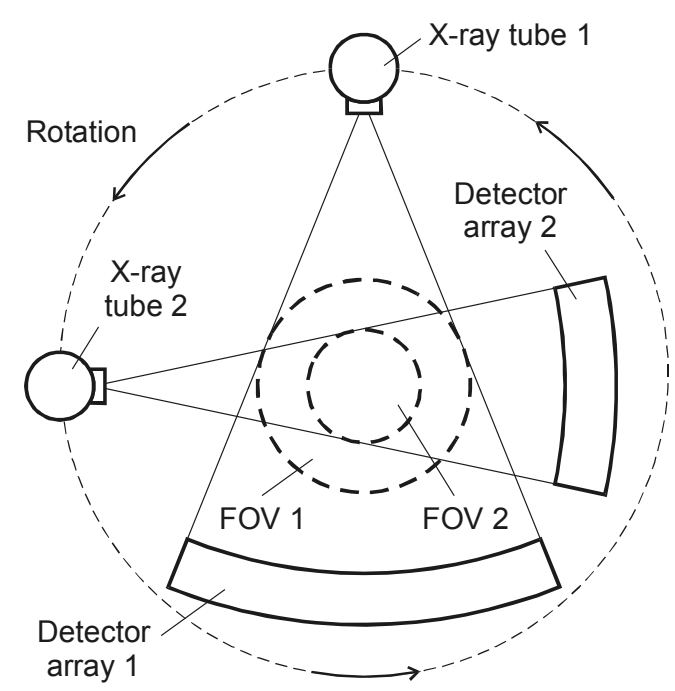

(d)

Figure 2.3. Novel CT techniques: (a) helical, (b) multislice, (c) cone-beam, and (d) dualsource. FOV is the field of view.

Source: Part (d) adapted with permission. W. A. Kalender, "X-ray computed tomography," Phys. Med. Biol. 51, R29-R43, 2006. 
gantry rotation. ${ }^{6}$ Typical values of the pitch lie between zero and two for single-slice helical scanners. ${ }^{8}$ A value less than one indicates overlapping of the projection data (a tight helix), whereas a value greater than one implies that a certain amount of the data is skipped (a loose helix). Due to the helical path of the x-ray tube around the patient, the backprojection reconstruction algorithm is modified to produce images that correspond to those acquired with a step-and-shoot CT scanner. This modification includes linear interpolation of the helical data set into a series of planar data sets. ${ }^{9}$ Because the location of each planar data set can be chosen arbitrarily within the scanned volume, reconstruction of almost an infinite number of slices is possible.

Multislice CT further increases the efficiency of step-and-shoot and helical scanners by adding several 1D detector arrays in the $z$ direction (Fig. 2.3-b) ${ }^{2,6}$ Such a design allows acquisition of multiple slices in a single rotation. This significantly reduces the scan time for acquiring volumetric data. When combined with helical scanning, multislice $\mathrm{CT}$ can be used to image larger volumes in a given time, or to image a given volume in a shorter time, compared with the single-slice scanners. ${ }^{8}$ In addition to reduced scan times, multislice CT offers improved cross-plane (z-axis) spatial resolution. Also, the use of multiple slices considerably enhances x-ray tube utilization. One potential problem in multislice CT is a higher level of scattered radiation per slice. Because scatter deleteriously affects image quality and radiation dose, multislice scanners employ an optimized, scatter-reducing collimator and detector design. Furthermore, for helical scanning, the $z$-axis sampling is optimized as well. Due to these optimizations, multislice helical CT scanners demonstrate up to $20 \%$ lower noise than their single-slice counterparts. ${ }^{6}$ Another distinct feature of multislice helical scanners is that their slice 
thickness can be chosen retrospectively after data acquisition. ${ }^{8}$ Such flexibility permits even more reconstruction choices. Current commercial multislice CT scanners typically have from 4 to 16 slices. ${ }^{2}$ The state-of-the-art machines incorporate as many as 64 slices, ${ }^{11}$ and the development of a 256 -slice clinical unit has been reported. ${ }^{12}$ The latter machine, also known as a wide-area detector CT scanner, can cover a whole organ in a single rotation and, therefore, may alleviate the need for helical scanning.

A logical extension of the multislice technique, with its several-row and wide-area detector arrays, is cone-beam CT, which uses a planar detector array (Fig. 2.3-c). In conebeam $\mathrm{CT}$, the 2D acquisition of the projection data all the way around a patient allows, in principle, reconstruction of many slices with only one gantry rotation and no translation of the patient in the $z$ direction. ${ }^{9}$ Cone-beam CT offers the largest x-ray tube utilization, but, obviously, suffers from more scattered radiation, which reduces the image quality. The conversion of the cone-beam, 3D data set into CT images is done by a backprojection-based, cone-beam reconstruction algorithm, which takes into account the x-ray beam divergence in both spatial dimensions. Because of yet high fabrication costs for planar detector arrays that are large enough for whole-body scanning (with submillimeter spatial resolution), cone-beam CT at present is mainly utilized in applications requiring smaller FOVs. Some of the most successful implementations of the cone-beam approach can be found in micro-CT.

The described developments in clinical CT allow examination of most organs and parts of the human body with very high reliability. One exception is cardiac imaging, which requires better temporal resolution, i.e., shorter scan times. Traditionally, scan times of mechanical CT scanners have been reduced by faster gantry rotation. A good 
example is helical and multislice scanners, whose rotation time has decreased from 0.5 to $0.33 \mathrm{~s}$ since their first introduction; ${ }^{11}$ the scan time of these machines has improved accordingly. There are, however, severe obstacles against further reduction in the rotation time. The main problems include increased centrifugal forces and excessive requirements for x-ray tube power. To decrease the scan time below the rotation-time limits, half-scan reconstruction algorithms have been proposed. ${ }^{13}$ These algorithms use only approximately 180 deg of projection data to reconstruct one image. Hence, when utilized in clinical CT scanners with 330-ms rotation time, the half-scan reconstruction algorithms permit the scan time of about $165 \mathrm{~ms}$. Unfortunately, such temporal resolution is still inadequate for successful cardiac imaging in many clinical cases.

Dual-source CT (DSCT) represents an efficient way to reduce the scan time even further, well beyond the current mechanical and x-ray power limits on the gantry rotation time. ${ }^{11}$ The first DSCT scanner, which became available in 2005 , is equipped with two $\mathrm{x}$ ray tubes and two corresponding multislice detector arrays, mounted onto a single gantry at 90-deg angles (Fig. 2.3-d) ${ }^{12,13}$ One detector array covers the entire FOV of $50 \mathrm{~cm}$, whereas the other detector array is restricted to a smaller, central FOV of $26 \mathrm{~cm}$. Because of the 90-deg angular offset between the x-ray tubes, only 90 deg of rotation is needed to acquire projection data over a 180-deg angle. Therefore, using half-scan reconstruction algorithms, it is possible to produce images with the scan time that corresponds to one quarter of the rotation time. The DSCT scanner has the 330-ms rotation time and, thus, achieves the scan time of $83 \mathrm{~ms}$. With such high temporal resolution, the heart and arteries can be imaged with almost no motion-induced artifacts, as has been shown by first clinical evaluations. The DSCT machine is also capable of dual-energy scanning, by 
operating the x-ray tubes at different energy levels. ${ }^{12}$ The dual-energy mode can offer better tissue characterization.

Today's state-of-the-art clinical CT scanners, which are mostly multislice helical third-generation machines, provide the in-plane spatial resolution of about $2.5 \mathrm{lp} / \mathrm{mm}$ and the cross-plane spatial resolution down to $0.5 \mathrm{~mm} .{ }^{11}$ Their contrast resolution approaches $0.3 \%$ ( $3 \mathrm{HU}$ ), and the typical radiation dose to the patient is around $30 \mathrm{mGy}$. With the gantry rotation time of $330 \mathrm{~ms}$, these scanners demonstrate the 165-ms (83-ms for DSCT) temporal resolution, which allows imaging of whole organs or the whole body in 5 to $20 \mathrm{~s}$.

\subsubsection{Micro-CT}

Micro-CT (or $\mu \mathrm{CT}$ ) is, in essence, a scaled-down version of clinical CT. MicroCT was developed in the early 1980s to overcome limited spatial resolution of clinical CT scanners when imaging small animals and biopsy-sized specimens from larger animals and humans. ${ }^{14}$ With the increasing interest in such imaging, micro-CT has rapidly evolved into an efficient minimally-invasive method for high-resolution studies of micro-anatomy; this method provides relatively short scan times, isotropic volume coverage, excellent sensitivity to skeletal tissue, and good sensitivity to soft tissue, especially when contrast-enhancing media are employed. ${ }^{15}$

A typical micro-CT scanner consists of a microfocus x-ray tube, a support for the object being imaged, and a high-resolution detector (Fig. 2.4). The microfocus x-ray tube has the focal-spot size in the range of 10-100 $\mu \mathrm{m}$ and usually incorporates a stationary tungsten anode. ${ }^{14-16}$ The high-resolution detector is either an x-ray image intensifier, as in 


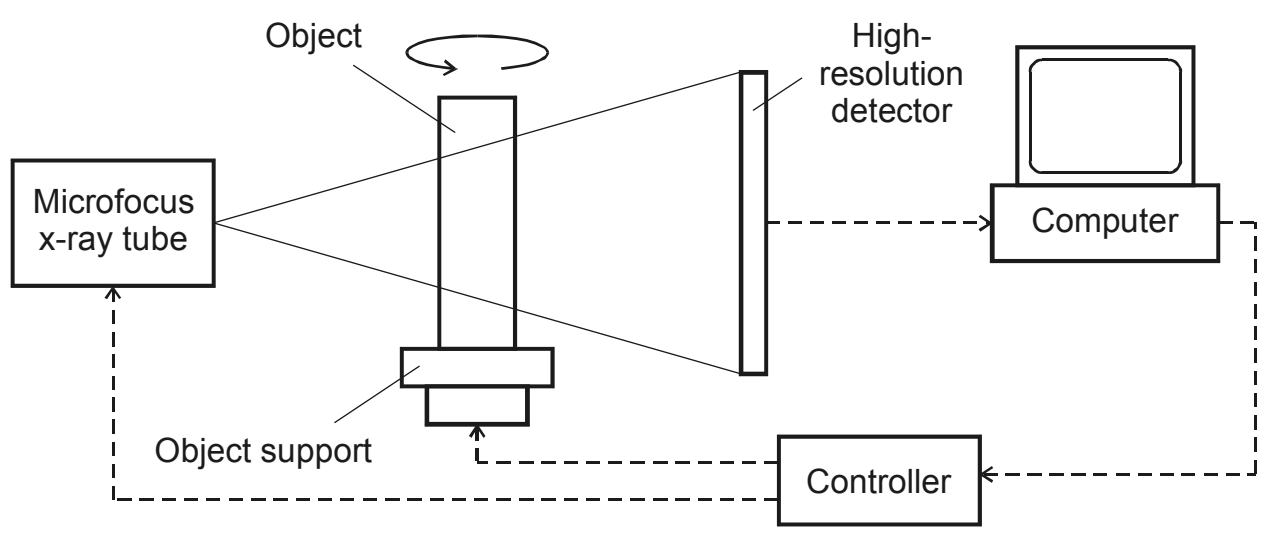

Figure 2.4. Typical micro-CT scanner.

Source: Adapted with permission from Elsevier. D. W. Holdsworth and M. M. Thornton, "Micro-CT in small animal and specimen imaging," Trends Biotechnol. 20, S34-S39, 2002. 
early micro-CT systems, or a scintillating screen optically connected (via a lens or a fiber-optic taper) to a charge-coupled device (CCD), as in the majority of current scanners. ${ }^{14,15}$ The use of a cooled CCD with fiber-optic coupling represents the most sensitive $\mathrm{x}$-ray detection approach available today. ${ }^{17}$ Some recent designs of micro-CT scanners utilize flat-panel arrays as the high-resolution detector. ${ }^{17-19}$ The pixel spacing of the detector is generally around $50 \mu \mathrm{m}$ or less. ${ }^{16}$ This spacing, along with the focal-spot size and system magnification, influences the scanner spatial resolution. The microfocus $\mathrm{x}$-ray tube and the high-resolution detector can be either stationary, with the rotating object, or mounted on a rotating gantry, with the fixed object. ${ }^{20}$ Projection data acquired by the detector are fed into a computer, where they are used to reconstruct a CT image. The scanner also includes a controller (or controllers) to assure proper operation of the $\mathrm{x}-$ ray tube and the rotating device.

As implied by the above description, the typical micro-CT scanner employs the cone-beam approach for data collection. This approach is preferable for micro-CT due to maximum x-ray tube utilization and much faster volumetric acquisition. ${ }^{21}$ Although the single-slice geometry is still used in less time-sensitive applications, the cone-beam approach seems to become a de facto standard in the area. In addition to providing volumetric coverage, the cone-beam approach serves as a means of achieving the system magnification needed to exceed the inherent spatial resolution of the detector. ${ }^{14,20}$ Because of the resulting 3D data set, micro-CT utilizes a cone-beam reconstruction algorithm. The original method, known as Feldkamp (or FDK) algorithm, is an approximation of 3D filtered backprojection. ${ }^{22}$ Despite recent introduction of many other methods, including exact solutions, the Feldkamp algorithm remains the most widely 
employed cone-beam reconstruction technique due to its straightforward implementation and applicability to practical systems. ${ }^{15}$

Several variations of micro-CT scanners have been developed. The described design, which is based on a conventional $\mathrm{x}$-ray tube source, is known as a bench-top micro-CT scanner. ${ }^{14}$ This design has been implemented in two configurations, for either in vivo or in vitro imaging. ${ }^{16}$ The bench-top micro-CT scanners for in vivo imaging are used to scan small animals. To avoid soft tissue distortions and movements during the scan, the animal in these systems is kept in a fixed horizontal position, while the gantry rotates around the animal, much like in clinical units. ${ }^{14,20,23}$ Because the in vivo scanners are primarily optimized for minimum radiation dose to the live animal, they have relatively low, as for micro-CT, spatial resolution, typically only $50-100 \mu \mathrm{m}$ isotropically. ${ }^{16}$ Their scan time, however, is rather short, usually less than $10 \mathrm{~min}$. The FOV of such scanners ranges from about 50 to $100 \mathrm{~mm}$. Due to modest spatial resolution, the in vivo systems are also referred to as mini-CT scanners.

The other configuration of bench-top micro-CT scanners, for in vitro imaging, is utilized to scan small specimens. In those systems, the specimen rotates around its vertical axis, but the $\mathrm{x}$-ray tube and detector are kept stationary. ${ }^{14,20,24}$ The in vitro scanners are generally optimized for maximum spatial resolution, which is chosen to approach the resolution of histological microscopy. As a result, such scanners reach isotropic spatial resolution of around $10-50 \mu \mathrm{m} .{ }^{14-16}$ Their typical scan time is $10-30 \mathrm{~min}$, and they commonly have the FOV of $15-50 \mathrm{~mm}$. For many applications, the bench-top micro-CT scanners for in vitro imaging offer a cost-effective alternative to traditional microscopy and histology methods when studying microstructure of biopsy-sized 
specimens.

Although some bench-top micro-CT scanners can achieve spatial resolution as high as $5 \mu \mathrm{m}$, even better resolution is provided by the synchrotron-based design. ${ }^{14}$ This design employs a monochromatic synchrotron x-ray source and a detector usually in the form of a scintillator-CCD couple. Because of the parallel x-ray beam geometry, the synchrotron-based micro-CT scanners cannot use the cone-beam approach to magnify the projection image. Instead, the magnification is accomplished by either optical coupling between the scintillator and the CCD, x-ray focusing via zone plates, or wavelengthspecific x-ray diffraction in a Bragg magnifier. ${ }^{14,20,25}$ The synchrotron-based micro-CT scanners have spatial resolution of $1 \mu \mathrm{m}$ or higher, allowing imaging of sub-cellular structures. ${ }^{25,26}$ Despite their superior resolution, the availability of such systems is limited, due to their dependence on synchrotron radiation sources.

As the size of an object being imaged and the voxel dimensions in micro-CT are much smaller compared with clinical scanners, several physics and technology aspects become important. First, because of the smaller object size, micro-CT requires lower xray photon energy, typically less than $25 \mathrm{keV} \cdot{ }^{14,27}$ In this energy range, $\mathrm{x}-$ ray photons interact with matter primarily via the photoelectric effect, whereas in clinical scanners, in which the photon energy generally exceeds $50 \mathrm{keV}$, the main interaction mechanism is Compton scatter. A desirable feature of the photoelectric effect is considerably higher and more atomic-number-dependent x-ray attenuation, which permits much better tissue discrimination in micro-CT. The drawback, however, includes stronger dependence of that attenuation on the photon energy. Due to such dependence, micro-CT scanners are very sensitive to the x-ray photon energy, which must be adjusted according to the object 
size to achieve a maximum signal-to-noise ratio (SNR). ${ }^{15,20,27} \mathrm{~A}$ further consequence of the strong energy dependence is higher susceptibility of micro-CT to beam-hardening artifacts; thus, nearly monochromatic radiation should be used to minimize these artifacts. Another important aspect of micro-CT scanners relates to their voxel dimensions being much smaller than in clinical systems. Smaller voxels require very efficient detectors, longer acquisition times, and higher radiation doses to achieve reasonable image quality. ${ }^{23,28-30}$ Also, because of the volumetric acquisition, smaller voxels in micro-CT lead to a huge amount of information (several gigabytes) in a typical data set. ${ }^{16,23,26}$ To store, process, and reconstruct this information, large computer resources and longer execution times are needed. The final aspect of micro-CT scanners is a result of scaling-down the system design. Due to a smaller size, all scanner components must be machined with higher accuracy, and all mechanical movements (especially in rotating-gantry scanners) must be performed with greater stability and precision. $^{14,23}$

In summary, micro-CT is a rapidly developing field stimulated by the increasing demand for small-animal and small-specimen imaging. At its current stage, micro-CT offers isotropic spatial resolution from about 100 to $10 \mu \mathrm{m}$ for bench-top scanners and even higher, up to sub-micrometer resolution for synchrotron-based systems. Micro-CT demonstrates high sensitivity to skeletal tissue and reasonably good sensitivity to soft tissue; the soft-tissue discrimination can be enhanced by a contrast agent. The scan times in micro-CT are comparable to those in other high-resolution imaging modalities. 


\subsection{Limitations of current CT scanners}

Despite being an extremely successful and technologically advanced biological imaging procedure, $\mathrm{CT}$ does have some drawbacks and limitations. The main downside of $\mathrm{CT}$ is the inherent use of ionizing radiation, which carries potential risks to patients, research animals, and operating staff. Although ionizing radiation can damage tissue in several distinct ways, the primary concern is the increased probability of cancer due to extensive and frequent radiation exposure during CT scans. In clinical settings, according to Table 2.1, the typical effective dose equivalent ranges from $3 \mathrm{mSv}$ for head $\mathrm{CT}$ to $10 \mathrm{mSv}$ for body CT. These values are high compared with natural background radiation, which is about $3 \mathrm{mSv}$ per year. ${ }^{31}$ Thus, it would take a person 3.3 years to get the same amount of background radiation that a clinical CT body-scan delivers in less than a minute. In micro-CT, radiation doses are even higher and often approach the lethal limits for small animals. Such elevated doses result from the concurrent requirements for high spatial resolution and a low noise level.

An important limitation of clinical CT scanners is their poor spatial resolution, which is much lower than that of screen-film projection radiography. As mentioned previously, the most advanced among these scanners can provide the in-plane spatial resolution of only $2.5 \mathrm{lp} / \mathrm{mm}$ and even lower cross-plane resolution. Because such machines are used primarily for whole-body imaging and have an FOV of 40-50 cm, a further increase in their spatial resolution is limited mostly by high fabrication costs of large-field discrete detectors with small pixel spacing. A related disadvantage involves the fact that clinical CT scanners provide no improvement in spatial resolution when imaging objects down to a few centimeters in diameter. Micro-CT scanners, on the other 
hand, are very appropriate for imaging small objects. Bench-top versions of these scanners have isotropic spatial resolution approaching $50 \mathrm{lp} / \mathrm{mm}$. Such high resolution results mainly from employing small-field discrete detectors with very small pixel spacing. However, the FOV of micro-CT scanners is typically only $1-10 \mathrm{~cm}$, and they cannot be used to image medium-size or large objects.

A further limitation includes the fact that clinical as well as micro-CT scanners are inherently more prone to image artifacts. Not all of these artifacts can be successfully corrected. One of the most serious artifacts is associated with imaging extremely dense objects, such as bone, metal inserts, or concentrated contrast media. Images of these objects can contain streaks with erroneous CT numbers. Another frequent artifact, especially in micro-CT, is beam hardening, which can appear in images as shading or fuzziness, resulting in unpredictable CT-number shifts. Numerous other artifacts can produce additional streaks, shades, rings, bands, and curvilinear patterns, all of which cause inaccuracies in signal values and, therefore, interfere with quantitative analysis in CT.

In addition, most clinical and micro-CT scanners require acquisition of $\mathrm{x}$-ray projections through the entire object, not a truncated portion, in order to reconstruct an image. This is true even if reconstruction of only a small region of the FOV is desired. Such a limitation, caused by the specifics of backprojection reconstruction algorithms employed in these scanners, forces the use of the full FOV every time a smaller part of the anatomy is studied. Obviously, acquiring the complete set of projection data leads to an unnecessary increase in radiation dose as well as acquisition and reconstruction times.

One inherent limitation of micro-CT scanners consists in their relatively low 
sensitivity to soft tissue. Because the SNR of these scanners depends largely on the number of detected x-ray photons, the scanners' sensitivity is ultimately limited by allowed radiation exposure. Also, due to reduced power of microfocus x-ray tubes typically utilized in such systems, some micro-CT scanners exhibit rather long acquisition times, in order to collect a sufficient number of x-ray photons.

The final drawback, which applies primarily to clinical CT, is that the scanning equipment is sophisticated, costly, and expensive to maintain. The rotational and translational motions involved in a scan require highly powerful, precise, and reliable mechanics. The precision and stability requirements become even stricter in micro-CT, where all the mechanical components are of a much smaller size. 


\section{CHAPTER 3. VARIABLE RESOLUTION X-RAY COMPUTED TOMOGRAPHY}

This chapter describes the main principles, advantages, limitations, realizations, potential applications, and development of VRX CT, which can be considered as an extension of conventional CT introduced in the previous chapter.

\subsection{Projective compression and a VRX detector}

Conventional CT is a proven method of biological x-ray imaging. Since its first introduction more than three decades ago, this method has experienced tremendous advancement in terms of both basic technology and new clinical applications. Despite impressive progress in many aspects of CT imaging, there is a growing demand for further improvements in the image quality, dose efficiency, and scan speed. In the area of image quality, an important parameter for which additional increases are highly desired is spatial resolution.

Spatial resolution of conventional CT scanners is limited by, among other factors, detector resolution. Because nearly all such scanners employ detectors composed of discrete cells, the detector resolution is largely determined by the physical size of the cells. The cell size of detectors used in modern clinical CT machines is typically larger than $250 \mu \mathrm{m}$. This results in the maximum spatial resolution for these scanners of only $2.5 \mathrm{lp} / \mathrm{mm}$, as discussed in the previous chapter. Further reduction in the cell size is expensive considering the fact that a huge number of cells and associated electronic channels are required to cover a large FOV $(40-50 \mathrm{~cm})$ of clinical machines. Micro-CT scanners, on the other hand, have a much smaller FOV (typically only a few centimeters) 
and, therefore, use reduced-field detectors with a very small cell size, less than $50 \mu \mathrm{m}$. The small cell size leads to significantly-higher spatial resolution, up to $50 \mathrm{lp} / \mathrm{mm}$ for bench-top micro-CT systems. However, there is an ultimate "physics" limit to which spatial resolution can be improved by reducing the physical cell size. This limit is imposed by the size of deposited energy clouds created by reabsorbed secondary photons and electrons. ${ }^{32}$ Due to overlapping of such clouds, nearby x-ray photons would not be resolved in conventional detectors even if the cells in a discrete detector were made arbitrarily small or a continuous detection medium were employed (Fig. 3.1-a).

A new detection principle called "projective compression" overcomes all the previously discussed limitations and has a great potential for CT imaging. ${ }^{32-34}$ According to this principle, either a continuous or discrete $\mathrm{x}$-ray detection medium is constructed so that virtual or real detector cells appear smaller and closer together in projection, as viewed from the $\mathrm{x}$-ray source, than their physical size and spacing would imply. Two basic implementations of projective compression include detector angulation and "stairstepping."

In the first implementation, a continuous or discrete detector is simply angulated with respect to the x-ray beam to achieve projective compression (Fig. 3.1-b). ${ }^{32}$ Systems employing this geometry improve spatial resolution by a factor of $1 / \sin (\theta)$, where $\theta$ is the angle between the detector surface and the x-ray beam direction. The minimum angle, i.e., the maximum spatial resolution, is limited by signal intensity and, ultimately, by $\mathrm{x}-$ ray reflection off the entrance surface of the detector. For diagnostic x-ray beam energies, however, the latter phenomenon becomes significant only at extremely small angles corresponding to a resolution boost by a factor of over 800 . 


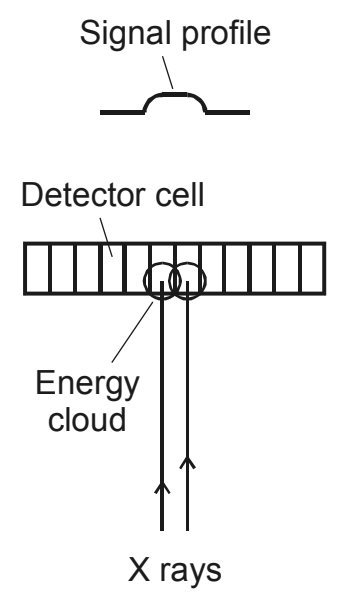

(a)

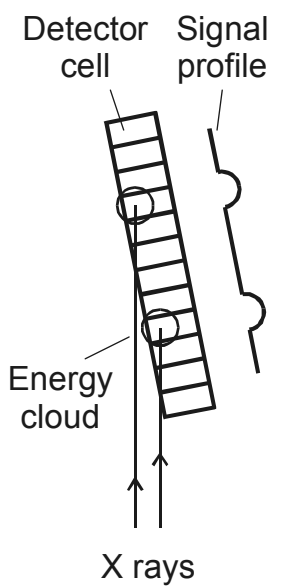

(b)

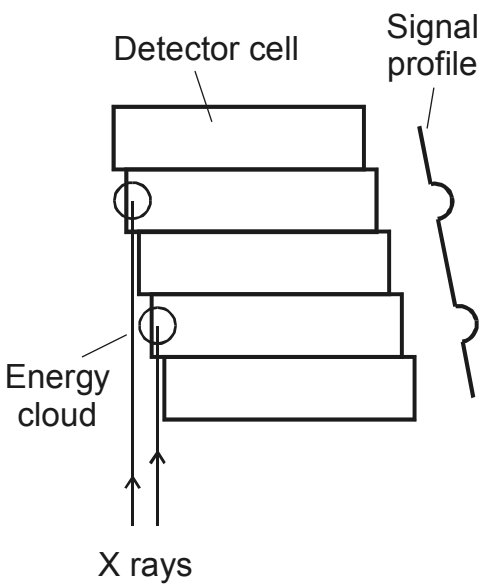

(c)

Figure 3.1. X-ray detector types: (a) conventional, (b) angulated VRX, and (c) stairstepped VRX.

Source: Adapted with permission. F. A. DiBianca, D. Gulabani, L. M. Jordan, S. Vangala, D. Rendon, J. S. Laughter, R. Melnyk, M. W. Gaber, and G. S. Keyes, "Fourarm variable-resolution x-ray detector for CT target imaging," Proc. SPIE 5745, 332-339, 2005. 
In the second implementation, projective compression is achieved by arranging detector cells like steps in a staircase (Fig. 3.1-c). ${ }^{32}$ For such a detector, spatial resolution is determined by the amount of the cell-to-cell offset, and reducing this offset increases the resolution. Here, no x-ray reflection occurs at all, in theory, and x-ray diffraction plays only a minor role. Thus, the main limitations of this geometry are signal intensity and engineering considerations.

A significant advantage of the detectors based on the projective compression principle is that they can be made variable in their FOV. ${ }^{32,35}$ For each object being imaged, the FOV is matched to the object size by changing either the detector angulation (Fig. 3.2) or cell-to-cell offset. As a result, spatial resolution of such detectors varies with the object size, and the highest-possible resolution is achieved for each particular object. Because of this property, projective compression was also labeled the "variable resolution X-ray" detection technique, and the corresponding detectors were named the VRX detectors.

Although a VRX detector can employ either implementation of the projective compression principle, the further discussion will be limited to angulated systems because they are simpler from the engineering point of view. Also, unless otherwise specified, only systems with a discrete detection medium will be considered.

\subsection{Advantages and limitations of the VRX detector}

The VRX detector offers several advantages for diagnostic and laboratory x-ray imaging. First, the detector provides increased spatial resolution. ${ }^{32-35}$ This resolution can exceed that of clinical CT detectors at large FOVs and, more importantly, is greatly 


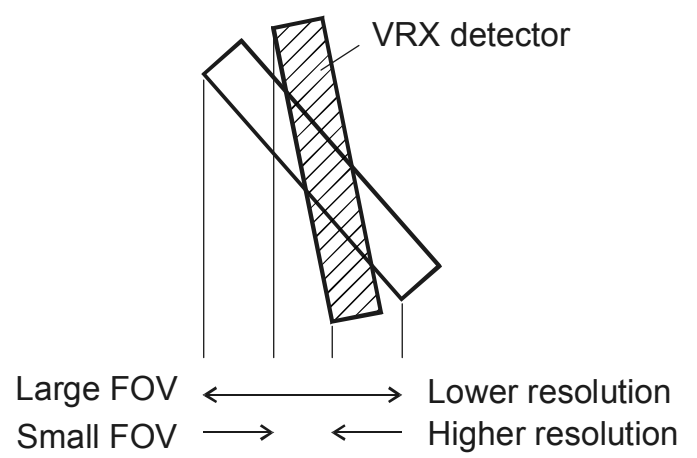

Figure 3.2. Spatial resolution of the VRX detector vs. its field of view (FOV).

Source: Adapted with permission. F. A. DiBianca, R. Melnyk, C. Duckworth, S. Russ, L. M. Jordan, and J. S. Laughter, "Comparison of VRX CT scanner geometries," Proc. SPIE 4320, 627-635, 2001. 
improved and approaches the resolution of micro-CT detectors at small FOVs. Such increased spatial resolution is a direct result of angulating the VRX detector with respect to the x-ray beam. Indeed, because of the angulation, the projected size and spacing of the detector cells are smaller than their physical size and spacing; the smaller cell size and spacing permit higher resolution.

Second, as mentioned previously, the spatial resolution and FOV of the VRX detector are variable. ${ }^{32,35}$ When the detector is angulated according to the object size, large as well as small objects can be imaged, and the highest-possible resolution is achieved at each particular FOV (Fig. 3.2). This is an improvement over clinical CT detectors, which are matched to the largest anatomical size expected and provide no increase in spatial resolution when imaging small objects. This is also superior to microCT detectors, which have a very limited FOV and cannot image even medium-size objects. Thus, the VRX detector spans both clinical CT and micro-CT domains of biological imaging and allows objects in a wide range of sizes (approximately $0.5-50 \mathrm{~cm}$ ) to be imaged with the maximum resolution.

Third, the spatial resolution of the VRX detector can be increased beyond the physics limit, imposed by the size of deposited energy clouds. ${ }^{32}$ This size is no longer the ultimate resolution limiter because in the angulated detector, as opposite to conventional detectors, the deposited energy clouds for nearby x-ray photons do not overlap even for an arbitrary small projected cell size, and the photons can be clearly resolved (Figs. 3.1-b and 3.1-c).

Fourth, for a relatively thin continuous detection medium, the quantum efficiency of the VRX detector improves as its spatial resolution increases. The reason for this 
improvement is, again, the detector angulation, which increases the effective thickness of the detection medium. Because of such an effect, thinner detection media can be used and better optical coupling can be accomplished.

Fifth, the VRX detector is well suited for the CT scanner geometry. ${ }^{32,35}$ This property results from the 1D nature of the resolution increase in the detector. Although the most obvious application of the VRX detector is a basic, single-slice step-and-shoot third-generation CT scanner, the corresponding technique can also be extended to multislice helical machines and cone-beam systems.

The VRX detector has several limitations as well. The most evident is a nonuniform data set, in terms of both spatial resolution and signal intensity. ${ }^{32}$ Such nonuniformity is a side-effect of the detector angulation, which causes the cell aperture, system magnification, and x-ray fluence to vary over the length of the detector. This variation exists only for diverging $\mathrm{x}$-ray beams, such as those produced by standard $\mathrm{x}$-ray tubes; it does not occur when parallel beams, i.e., those from synchrotrons, are utilized. In the case of diverging x-ray beams, the non-uniform data set can be partially corrected at the pre-processing and reconstruction stages.

The next disadvantage of the VRX detector is the increased system magnification for the cells farther, due to the detector angulation, from the $\mathrm{x}$-ray tube. ${ }^{32,35}$ The increased system magnification enlarges the projected size of the tube focal spot. This limits the overall spatial resolution of the system and, therefore, diminishes the main benefit of the VRX detector. For that reason, the smallest possible focal spots must be used when employing standard x-ray tubes with diverging beams. Again, for parallel x-ray beams, no such problem exists. 
Another limitation is a low SNR of the VRX detector at high spatial resolution. The low SNR results from a small projected area of the detector cells and, thus, a limited number of $\mathrm{x}$-ray photons incident on each cell when the detector is placed at a small angle with respect to the x-ray beam. As mentioned in the previous chapter, this limitation is inherent to all high-resolution detectors with a reduced cell size. Similarly to those devices, an acceptable SNR in the VRX detector can be achieved by prolonging the acquisition time, using a high-sensitivity detection technology, and utilizing a high-output $\mathrm{x}$-ray tube, if possible.

A further drawback of the VRX detector is increasing x-ray energy escape from the detector cells as the angle between the detector surface and the x-ray beam decreases. ${ }^{32}$ The incident $\mathrm{x}$-ray energy escapes from the sides of the cells (via primary photons) and from their front surface (via secondary photons and electrons). The side escape is due to $\mathrm{x}$-ray photons traversing the cells at an angle. At smaller angles, a shorter fraction of a cell is traversed, and more x-ray photons pass through the cell without being absorbed. This type of the energy escape gives rise to inter-cell x-ray cross-talk, ${ }^{36}$ when x-ray photons incident on one cell are absorbed in the neighboring cells. The inter-cell xray cross-talk can be reduced by placing $\mathrm{x}$-ray attenuating separators between the detector cells. The other type of the $\mathrm{x}$-ray energy escape, the front escape, increases at smaller angles because $\mathrm{x}$-ray interactions take place closer to the detector front surface. In this case, however, the fraction of the escaping energy never exceeds $50 \%$, as a result of the isotropic spatial distribution of scattered photons and electrons. Also, both types of the $\mathrm{x}$ ray energy escape will generally be lower at small angles during normal operation of the VRX detector because lower $\mathrm{x}$-ray energy is required for imaging small objects. 
An additional limitation is the difficulty of using a standard anti-scatter collimator

with the VRX detector. ${ }^{32,35}$ Such a collimator usually includes inter-cell septa attached to the detector front surface. This design is hard to implement in the VRX detector because, as the detector is angulated, the septa must be counter-pivoted very accurately, to avoid any significant blocking of the x-ray beam. Even if the septa are precisely pivoted, they will block the x-ray beam at small angles, when the septum thickness exceeds the projected size of a detector cell. Fortunately, at small angles, a low scatter-to-primary ratio due to low x-ray energy may obviate the need for anti-scatter collimation. At large and medium angles, however, one possible solution is to use detached inter-cell septa placed in front of the detector and set at some angle relative to the scan plane. Another solution, which works at any angle, involves replacing the standard, multi-septum collimator with a post-patient beam-envelope collimator.

The described limitations of the VRX detector do not undermine its great potential for improving and varying spatial resolution in biological x-ray imaging, particularly CT. With its clear advantages, the VRX detector is a very promising imaging device. Its performance, therefore, should be thoroughly evaluated, and its benefits should be further developed.

\subsection{Single-arm, dual-arm, and four-arm VRX detectors}

Several configurations of the VRX detector have been proposed. They are often referred to as single-arm, dual-arm, and four-arm VRX detectors. These configurations can be conveniently described from the CT perspective.

In a simplest, single-arm configuration (Fig. 3.3-a), the VRX detector includes 

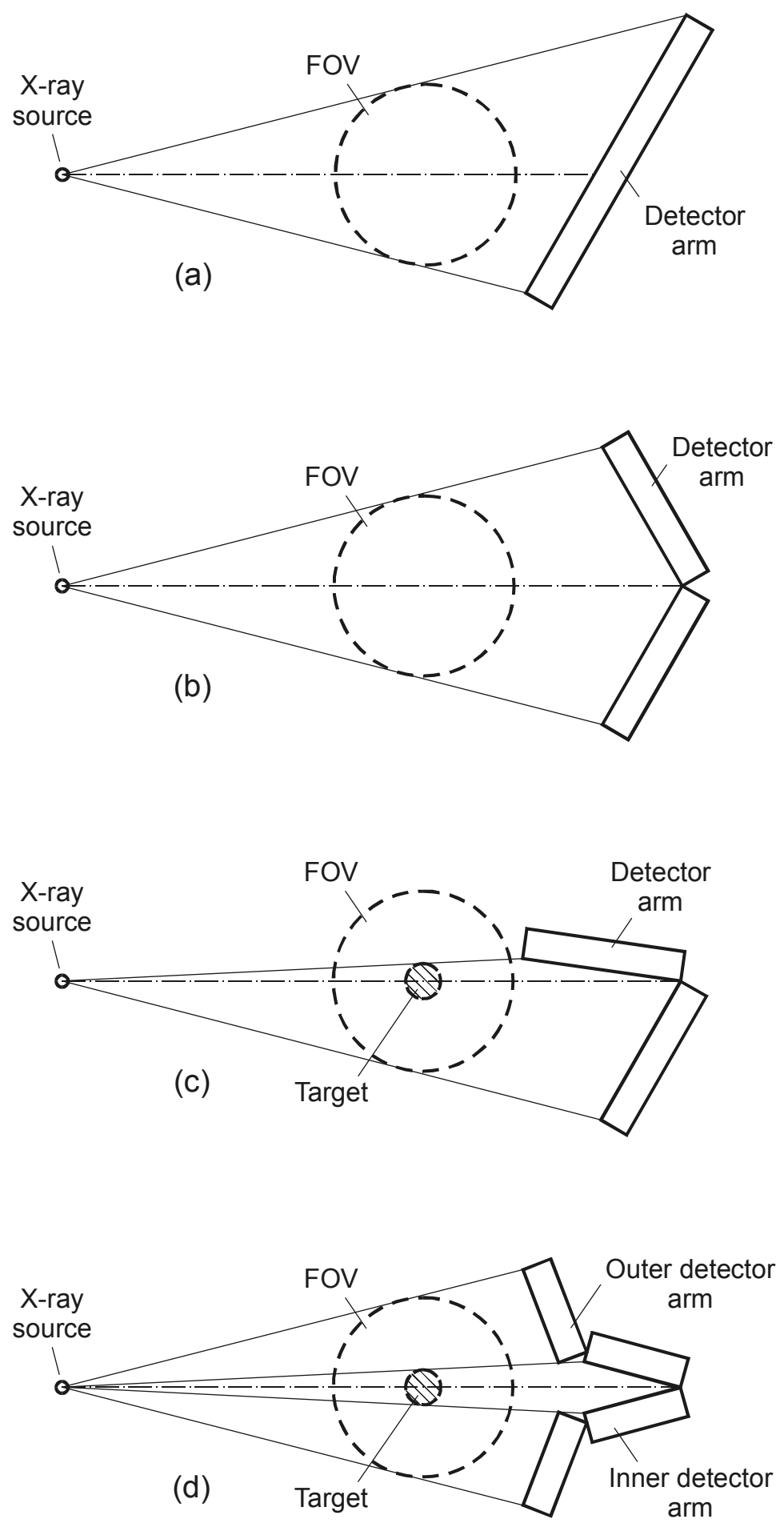

Figure 3.3. Configurations of the VRX detector: (a) single-arm, (b) symmetrical dualarm, (c) asymmetrical dual-arm, and (d) four-arm. FOV is the field of view. 
one scintillator-photodetector array (one arm). ${ }^{32,37}$ This arm can rotate around a pivotal point usually located on the front surface of the scintillator, half-way between the detector ends, although other arrangements are possible. By rotating the arm, the detector spatial resolution can be varied according to the FOV.

A dual-arm VRX detector consists of two scintillator-photodetector arrays (two arms) with a common pivotal point (vertex) ${ }^{37}$ In this configuration, the arms can be placed either symmetrically (Fig. 3.3-b) or asymmetrically (Fig. 3.3-c) around the detector centerline, which is the line connecting the x-ray source and the detector vertex. In the symmetrical (or "normal") mode, both arms provide the same increase in spatial resolution and are always angulated equally to cover the full FOV. In the asymmetrical (or "target") mode, only one arm (low-resolution arm) is angulated according to the FOV. The other arm (high-resolution arm) is set at a smaller angle with the detector centerline and covers just a small target region inside the FOV during a CT scan. This latter region is imaged with higher spatial resolution than the rest of the object. Thus, the asymmetrical mode of the dual-arm VRX detector allows "target imaging" and offers an additional resolution improvement for the target, compared with the basic VRX detection technique. The asymmetrical mode can use several arrangements of the detector arms. ${ }^{38}$ Those arrangements differ mainly by how much of the FOV and target (e.g., half-FOVhalf-target or half-FOV-full-target) is covered in a single projection by the low- and highresolution arms, respectively. In both symmetrical and asymmetrical modes, the dual-arm VRX detector can be easily converted to the single-arm configuration by opening the arms to form a 180-deg angle between each other.

A further development is a four-arm VRX detector (Fig. 3.3-d), specifically 
designed for target imaging. ${ }^{38,39}$ Such a detector includes two inner and two outer arms. The inner arms are pivoted around the vertex, much like in the dual-arm configuration. The outer arms are placed at the ends of the inner arms, at angles with the detector centerline that are larger than those for the inner arms. The outer arms do not have any fixed pivotal points; when angulated, these arms rather "slide" along the sides of the inner arms and are carefully aligned to avoid blocking any $\mathrm{x}$ rays incident on the inner arms. Both pairs of the arms are usually positioned symmetrically around the detector centerline, so that the inner arms cover a small target region, while the outer arms scan the rest of the FOV. Due to larger angulation (smaller angles with the detector centerline) for the inner arms compared with the outer arms, the target is imaged with higher spatial resolution than the rest of the object. Again, the four-arm VRX detector can be easily transformed to the dual-arm or single-arm configuration.

Each of the three configurations of the VRX detector has its advantages and limitations. An evident benefit of the single-arm configuration is the absence of any gaps or discontinuities in the detector. ${ }^{37}$ Also, there is no x-ray scatter from the opposite arm because the left and right halves of the detector lie in the same plane. One disadvantage of the single-arm configuration consists in distinct asymmetry between the left and the right detector halves. Another drawback includes a more non-uniform data set due to larger variations in the detector aperture, sampling distance, inter-cell x-ray cross-talk, and system magnification from one end of the detector to the other. Additionally, the single-arm configuration has higher median system magnification and a larger depth in the $\mathrm{x}$-ray beam direction.

The dual-arm VRX detector, when used in the normal mode, provides obvious 
advantages of left-right symmetry and smaller variations in the detector parameters over the length of the detector. ${ }^{37}$ Hence, the normal-mode dual-arm configuration produces a more uniform data set than that from the single-arm detector. When in the target mode, the dual-arm configuration loses its symmetry but allows an additional spatial resolution increase for the target. In any mode, the dual-arm VRX detector yields lower median system magnification and a shorter depth in the x-ray beam direction compared with the single-arm configuration. The limitations of the dual-arm detector include inter-arm x-ray scatter (especially at small angles) and a central dot artifact due to a physical gap at the detector vertex. Despite these drawbacks, the dual-arm configuration appears preferable to the single-arm detector for "non-target imaging."

The main advantage of the four-arm configuration of the VRX detector is that it combines both left-right symmetry and suitability for target imaging. ${ }^{39}$ Also, because such a configuration can be easily converted to the dual-arm or single-arm detector, the four-arm design offers the most imaging choices. However, the four-arm configuration has several downsides as well. Relative to the dual-arm detector, the four-arm configuration shows larger variations in the detector and system performance from one end of the detector to the other, higher x-ray scatter between the inner arms, and more gaps or discontinuities in the detector. A further limitation results from the fact that an approximate mathematical method is used to reconstruct the target with extra-high spatial resolution. It is not clear at this time whether the four-arm configuration is more suitable for target imaging than the asymmetrical-mode dual-arm detector. Recent comparative modeling of these configurations has revealed slightly lower performance of the four-arm detector. ${ }^{38}$ Additional studies are needed to choose the best VRX detector configuration 
for target imaging.

\subsection{VRX CT scanner and its potential biomedical applications}

A typical VRX CT scanner (Fig. 3.4) includes a dual-arm VRX detector that operates in the normal mode. ${ }^{36}$ Because the main application of the typical scanner is non-target imaging, the normal-mode dual-arm detector configuration ideally suits this purpose. According to the previous section, such a configuration provides left-right symmetry, low system magnification, small variations in the detector performance from one end of the detector to the other, and a compact system design. The typical VRX CT scanner also includes an x-ray source and support for an object being imaged. The maximum size of the object determines the scanner FOV. The x-ray source and detector are rotated around the object to make a CT scan.

The VRX CT scanner offers unique possibilities for improving spatial resolution in biological x-ray imaging. When the arms of the dual-arm VRX detector are set at maximum angles with the detector centerline, the scanner has the largest FOV (which can be matched to that of clinical CT machines) and the lowest spatial resolution (which can be similar to or higher than the resolution of clinical units). This regular-resolution mode is used to image large anatomical regions, such as chests or abdomens of most patients. Whenever smaller anatomical regions (heads, necks, extremities, etc.) or small (pediatric) patients are scanned, the detector arms are pivoted to smaller angles, allowing a corresponding increase in spatial resolution. In this intermediate-resolution mode, the VRX CT scanner fills the gap between clinical and micro-CT machines. To image even smaller objects, such as small animals and biopsy-sized specimens, the arms of the VRX 


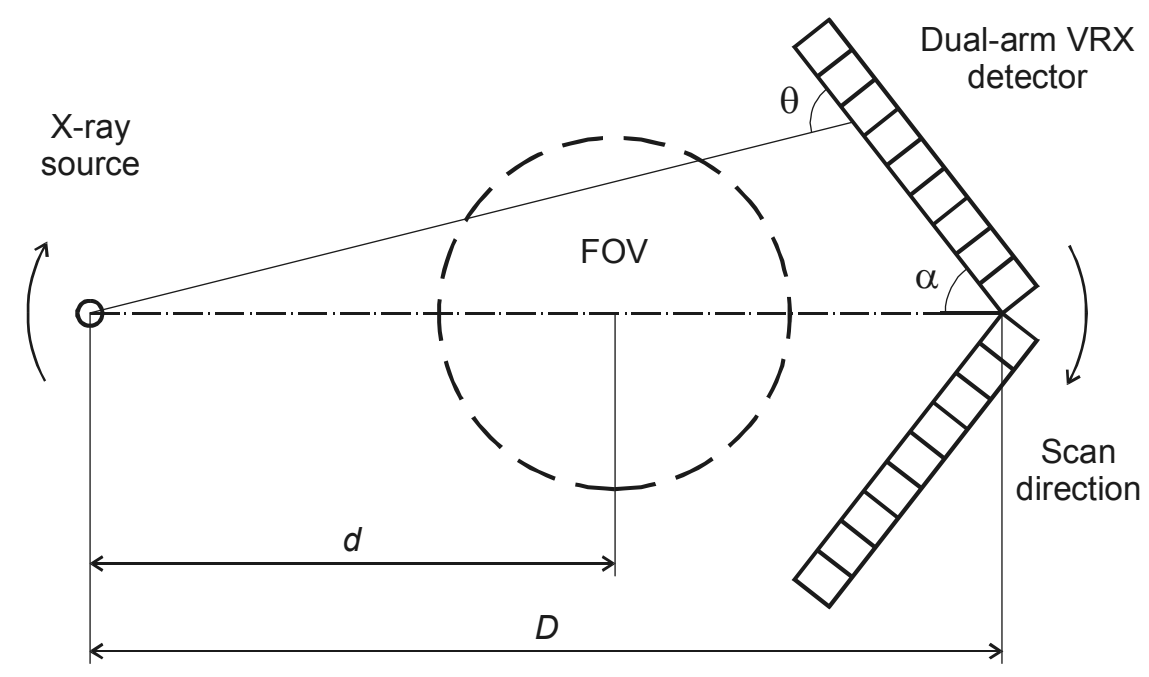

Figure 3.4. Typical VRX CT scanner: $D$ - source-vertex distance, $d$ - source-object distance, $\alpha$-opening half-angle, and $\theta$-incident angle. FOV is the field of view.

Source: Adapted with permission. R. Melnyk and F. A. DiBianca, "Monte Carlo study of $\mathrm{x}$-ray cross-talk in a variable resolution x-ray detector," Proc. SPIE 5030, 694-701, 2003. 
detector are placed at very acute angles (about 1-5 deg) with the detector centerline. This results in the maximum spatial resolution, which can approach that of micro-CT systems. Thus, by angulating the detector arms according to the size of the object being imaged, the spatial resolution of the VRX CT scanner is varied in such a way that the highestpossible resolution is provided for each particular object.

Because the VRX CT scanner spans both clinical and microscopy domains of biological imaging, the range of the scanner's potential applications is rather extensive. On one hand, the VRX CT scanner can be used to diagnose the same medical conditions whose detection is traditionally done by clinical machines. An important example of such applications includes examination of head trauma, which requires imaging of possible skull fractures, underlying brain damages, or hemorrhage. ${ }^{8}$ Another critical application is detection of brain tumors, by either visualizing calcifications or studying, with the help of contrast agents, the blood flow. The VRX CT scanner can also be useful in diagnosis of pulmonary diseases, particularly diffuse lung diseases such as silicosis, fibrosis, and emphysema. An additional application involves abdominal imaging, to visualize compound bone fractures as well as to detect, mostly via contrast media, abdominal tumors and ulcerations in the liver. In all these applications, the VRX CT scanner can out-perform clinical machines by providing better spatial resolution and the ability to match the resolution to the object size.

On the other hand, the VRX CT scanner can be applied to numerous diagnostic and laboratory tasks from the micro-CT domain. The primary application in this area is small-animal (in vivo) and human-specimen (in vitro) bone imaging, which entails accurate measurements of changes in bone architecture, density, and mineralization due 
to osteoporosis and osteoarthritis. ${ }^{16}$ A related task that can also benefit from the use of the VRX CT scanner is in vitro dental imaging, which includes non-destructive assessment of internal and external tooth morphology, 3D analysis of root canal geometry, and visualization of changes during endodontic treatment. ${ }^{20}$ An additional application from the micro-CT domain involves whole-animal imaging of genetically altered small animals, to evaluate phenotype changes in skeletal and soft (muscle or fat) tissues when developing new drugs and therapies for various human diseases. ${ }^{16}$ The VRX CT scanner can also be used in small-animal cancer research, for in vivo imaging of brain, prostate, lung, and bone tumors whose contrast is enhanced by appropriate contrast agents. ${ }^{15}$ Another possible application focuses on histological studies, where internal structure of excised human or animal specimens can be examined without any need for specimen preparation, sectioning, or staining and, hence, without any damage to the specimens. ${ }^{14}$ Additionally, the VRX CT scanner has potential for in vitro vascular imaging, to investigate microvasculature of large-animal specimens or sacrificed small animals injected with novel contrast media. ${ }^{16}$ Some other prospective tasks include quantification of administered radionuclides in small-animal nuclear imaging and localization of specific molecules in biopsy-based molecular analysis. ${ }^{14}$ In all these applications from the micro-CT domain, the VRX CT scanner can provide an advantage of a larger and variable FOV, which can be adjusted to the object size.

Finally, due to the unique ability of the VRX CT scanner to attain any, in principle, spatial resolution and FOV between the typical limits set by clinical and microCT systems, the scanner is very appropriate for many biomedical applications that, first, require better spatial resolution than that provided by clinical machines but, second, are 
too large to fit within the FOV of micro-CT units. Using the VRX CT scanner, one can image smaller patients (e.g., children) and small regions of interest (e.g., head, neck, upper and lower extremities, even fingers) with improved spatial resolution, to examine various traumas, study skeletal and soft tissues, diagnose calcification-producing pathologies, identify tumors, investigate various parts of anatomy, evaluate blood flow, do implant or surgical planning, etc. At the same time, larger animals or large-size specimens can be scanned, with only a slight loss of spatial resolution, for bone structural changes, dental and maxillofacial analysis, tumor growth, phenotype evaluation, histological studies, treatment progression, microvasculature assessment, and other purposes.

In summary, the VRX CT scanner can be developed into a highly versatile imaging device, with a wide range of potential applications. The scanner can also give rise to local, as opposite to the whole-body, imaging.

\subsection{Development of an experimental VRX CT scanner}

Since the introduction of the VRX detection technique in $1998,{ }^{33}$ a research effort on applying this technique to $\mathrm{CT}$ imaging has been taking place in the department of Biomedical Engineering and Imaging at the University of Tennessee Health Science Center. The goal of the research is to develop an experimental VRX CT scanner that would demonstrate the benefits of the proposed concept for clinical uses.

The development of the VRX CT scanner has been proceeding as follows. After establishing the feasibility of the VRX detection technique with a theoretical analysis and experimental data from a 16-channel solid-state detector ${ }^{32-34}$ as well as from a storage- 
phosphor plate, ${ }^{34,35}$ a 576-channel solid-state dual-arm VRX detector has been built and has shown promising results in terms of the signal level, SNR, and spatial resolution. ${ }^{40} \mathrm{~A}$ combined analytical and Monte Carlo study of this latter detector has confirmed the advantages of the dual-arm configuration compared with a single-arm detector. ${ }^{37}$ Therefore, the 576-channel solid-state dual-arm detector has been used to construct an experimental VRX CT scanner. Initial assessment of the scanner has included a Monte Carlo study of the x-ray cross-talk and has indicated the need for anti-scatter collimation and optimization of the $\mathrm{x}$-ray tube voltage. ${ }^{36}$ Further evaluation of the scanner parameters has become possible after development of an accurate reconstruction algorithm specific to VRX CT. Such an algorithm has been originally derived for a storage-phosphor system ${ }^{35,41}$ but has been later adapted to the experimental VRX CT scanner. As part of achieving high-quality reconstruction, a method to calibrate the experimental scanner by scanning an off-axis metal pin has been established. ${ }^{42}$ Recently, a four-arm VRX detector has been fabricated and preliminarily tested, showing potential for CT target imaging. ${ }^{39}$ As already mentioned, the four-arm configuration has been compared, by modeling, with the asymmetrical-mode dual-arm detector, also capable of target imaging, and the results have demonstrated slightly better performance of the latter, simpler configuration. ${ }^{38}$ The latest development includes implementation of the VRX detection technique in a conebeam CT system based on an indirect-conversion flat-panel array. ${ }^{43}$ This system has also exhibited an increase in spatial resolution, although the increase has been lower than that provided by the experimental scanner.

The experimental VRX CT scanner, which employs a 576-channel solid-state dual-arm detector, is the primary focus of the current study and will be considered in this 
dissertation. The experimental scanner has been constructed according to the typical diagram (Fig. 3.4), except an object being imaged is placed on a rotary table and rotates between the x-ray tube and the VRX detector, both of which are stationary. ${ }^{44}$ A detailed description of the design and parameters of the experimental VRX CT scanner will be given in Chapter 5.

An important step in the development of the experimental VRX CT scanner is a comprehensive evaluation of the scanner's performance. In general, the imaging system performance is evaluated in terms of spatial resolution, noise properties, and transfer of noise through the system. Because the main purpose of the VRX CT scanner is to improve spatial resolution, the initial phase of the scanner evaluation should focus on the assessment of this resolution. Until now, spatial resolution of the experimental VRX CT scanner has been only preliminarily evaluated by very approximate methods such as analytical simulation of the detector aperture and visual examination of simple test images. These approximate methods do not quantify various factors affecting spatial resolution of CT scanners in general (focal spot, detector design, reconstruction algorithm, etc.), nor do they accurately account for a range of effects specific to the VRX CT scanner (data non-uniformity, inter-arm scatter, varying system magnification, etc.). Also, the preliminary evaluation does not provide enough data on the spatial-resolution limits imposed by the chosen detector design, scanner geometry, available x-ray flux, and possible alignment errors. To account for all these factors and to assess practically achievable limits, a comprehensive evaluation of spatial resolution of the experimental VRX CT scanner is, therefore, required. Such an evaluation has been the subject of the current study and will be described in the next chapters. 


\section{CHAPTER 4. GENERAL THEORY AND METHODS FOR MEASUREMENT OF SPATIAL RESOLUTION}

This chapter outlines the common approach to describing spatial resolution, gives a comprehensive overview of the proper measures and their usage, defines the main components of spatial resolution, and explains in detail the measuring techniques for different types of imaging systems. The presented material provides background information for the subsequent discussion of the methods and results of the current study.

\subsection{Transfer-theory approach to spatial resolution}

Spatial resolution of an imaging system describes the system's ability to resolve closely placed objects. In the simplest way, spatial resolution can be specified as the minimum distance between two high-contrast objects that are still resolvable. This description, however, is not very practical because it depends to some degree on the shape of the objects being imaged. ${ }^{45}$ More useful measures of spatial resolution can be obtained by analyzing the system response. A theoretical basis for such analysis is given by transfer theory.

In transfer theory, an imaging system is considered in terms of the input-output relationship, without referring to the internal structure of the system. Typically, the input is an object being imaged, and the output is a resulting image. Both the input and output are represented by $2 \mathrm{D}$ functions in general. The values of the input function give the radiation intensity distribution incident on a detector after being attenuated by the object. Similarly, the values of the output function describe the distribution of pixel intensities in 
the image. The input and output usually reside on different planes and, therefore, are functions of different spatial variables. ${ }^{46}$ If $f(\xi, \eta)$ is the input defined in the object plane with the spatial coordinates $(\xi, \eta)$, and $g(x, y)$ is the output specified in the image plane with the spatial coordinates $(x, y)$, then the relationship between the input and the output of an imaging system can be mathematically written as

$$
g(x, y)=L\{f(\xi, \eta)\}
$$

where $L\{\}$ is the system operator.

The use of transfer theory requires an imaging system to be linear and shiftinvariant. Linearity includes two basic properties, superposition and scaling. ${ }^{10,47}$ Superposition means that the image of a sum of several inputs is equal to the sum of the images produced separately by each input. Scaling simply states that multiplication of an input by any real number corresponds to multiplication of the image by the same number. These two properties of a linear system can be combined into the following expression:

$$
L\left\{a_{1} f_{1}(\xi, \eta)+a_{2} f_{2}(\xi, \eta)\right\}=a_{1} L\left\{f_{1}(\xi, \eta)\right\}+a_{2} L\left\{f_{2}(\xi, \eta)\right\},
$$

where $a_{1}$ and $a_{2}$ are arbitrary real numbers. The second transfer-theory requirement, shift-invariance, refers to the ability of an imaging system to produce, for a given object, an image that has the same functional form regardless of the object location in the object plane. Shift-invariance is also called isoplanatism and can be formally expressed as

$$
g(x, y ; \xi, \eta)=g(x-\xi, y-\eta)
$$

for all image positions $(x, y)$ and object locations $(\xi, \eta) .{ }^{48}$

Both linearity and shift-invariance are idealizations; no actual imaging system strictly satisfies these requirements. However, because the transfer-theory approach provides such valuable measures of spatial resolution, practical imaging systems are often 
approximated to be linear and shift-invariant, in order to analyze them by means of transfer theory. Thus, many non-linear imaging systems can be linearized with an appropriate calibration or examined only within a signal region where the system response is linear. ${ }^{49}$ Similarly, imaging systems that are not shift-invariant over the entire object plane - as it is frequently the case - can be analyzed in smaller sub-regions of this plane over which the system response is approximately shift-invariant. These latter subregions are referred to as isoplanatic patches. ${ }^{47,48}$

In addition to linearity and shift-invariance, the transfer-theory approach assumes a "noise-free" imaging system as well as high contrast of objects used to measure spatial resolution. ${ }^{48-50}$ The former criterion is difficult to fulfill exactly because all actual imaging systems always contain some noise. In the presence of noise, transfer theory describes only the expectation values of the corresponding system parameters but ignores any statistical effects. ${ }^{49}$ If needed, these effects should be analyzed separately using the theory of stochastic processes. ${ }^{48}$ In practice, the noise-free requirement can be approximated by averaging a large number of quasi-identical images.

An important tool of transfer theory is Fourier analysis. According to the basic principle of this analysis, any well-behaved function of one or more variables can be represented as a sum of an infinite number of sinusoidal components of various spatial frequencies. ${ }^{47,48}$ By providing such a representation, Fourier analysis allows an alternative description of an imaging system in terms of spatial frequencies, in addition to the conventional system description in terms of spatial coordinates. The two descriptions - in the spatial-frequency domain and in the spatial domain - are equally valid and contain the same information. ${ }^{47,50}$ However, the spatial-frequency description is often 
more convenient because it simplifies calculations and offers additional insight into the system performance. ${ }^{46-49}$ The translation from the spatial into the spatial-frequency domain is done using the direct Fourier transform. ${ }^{10}$

$$
G(u, v)=\mathcal{F}\{g(x, y)\}=\int_{-\infty}^{\infty} \int_{-\infty} g(x, y) e^{-i 2 \pi(u x+v y)} d x d y
$$

whereas the opposite conversion is performed by means of the inverse Fourier transform

$$
g(x, y)=\mathcal{F}^{-1}\{G(u, v)\}=\int_{-\infty}^{\infty} \int_{0}^{\infty} G(u, v) e^{i 2 \pi(u x+v y)} d u d v
$$

In these expressions, $u$ and $v$ are spatial frequencies in the $x$ and $y$ directions, respectively. The spatial frequencies are typically given in $1 / \mathrm{mm}$ or cy/mm.

\subsection{Measures of spatial resolution}

In transfer theory, spatial resolution of an imaging system can be specified in either the spatial domain or the spatial-frequency (or, simply, frequency) domain. In each domain, several important measures of spatial resolution can be established. Despite different functional forms, the spatial-domain measures and the frequency-domain measures are closely related and can be converted from one to other by the Fourier transform.

\subsubsection{Spatial-domain measures}

In the spatial domain, spatial resolution of an imaging system is described by such transfer characteristics as the point spread function, line spread function, and edge spread function. These measures indicate how infinitely small point objects are transformed by the system into images with non-infinite dimensions. ${ }^{50}$ 


\section{$\underline{4.2 .1 .1 . ~ P o i n t ~ s p r e a d ~ f u n c t i o n ~}$}

The first spatial-domain descriptor of spatial resolution is the point spread function (PSF). By definition, the PSF represents a distribution of pixel intensities in the image of an infinitely small aperture (point source) of unit intensity. ${ }^{47}$ In an ideal imaging system, this distribution would correspond to a point in the image plane. In practical systems, however, the intensity distribution is smeared out around the ideal point and, therefore, forms a blurred image of the point source. The PSF provides a measure of this blurring. $^{47}$

Mathematically, the PSF is an image of a delta function $\delta(\xi, \eta)$,

$$
\operatorname{PSF}(x, y)=L\{\delta(\xi, \eta)\}
$$

The delta function has several important properties, one of which - the sifting property allows decomposition of any object into an array of point sources (2D delta functions): ${ }^{7,10,46}$

$$
f(\xi, \eta)=\int_{-\infty}^{\infty} \int^{\infty} f\left(\xi^{\prime}, \eta^{\prime}\right) \delta\left(\xi-\xi^{\prime}, \eta-\eta^{\prime}\right) d \xi^{\prime} d \eta^{\prime},
$$

where $f\left(\xi^{\prime}, \eta^{\prime}\right)$ is the point-source intensity proportional to the object attenuation at the position $\left(\xi^{\prime}, \eta^{\prime}\right)$. According to Eq. 4.1, the resulting image is given by

$$
g(x, y)=L\left\{\int_{-\infty}^{\infty} \int_{-\infty} f\left(\xi^{\prime}, \eta^{\prime}\right) \delta\left(\xi-\xi^{\prime}, \eta-\eta^{\prime}\right) d \xi^{\prime} d \eta^{\prime}\right\} .
$$

Considering $f\left(\xi^{\prime}, \eta^{\prime}\right)$ in this equation as a weighting factor for each delta function and treating the integral as a generalized sum, one can apply the linearity property (Eq. 4.2) and get the following expression: 


$$
g(x, y)=\int_{-\infty}^{\infty} \int_{0} f\left(\xi^{\prime}, \eta^{\prime}\right) L\left\{\delta\left(\xi-\xi^{\prime}, \eta-\eta^{\prime}\right)\right\} d \xi^{\prime} d \eta^{\prime}
$$

This expression can be rewritten using the definition of the PSF (Eq. 4.6) and, for convenience, changing the variables,

$$
g(x, y)=\int_{-\infty}^{\infty} \int_{0} f(\xi, \eta) \operatorname{PSF}(x, y ; \xi, \eta) d \xi d \eta
$$

When the shift-invariance property (Eq. 4.3) is also taken into account, the latter equation becomes

$$
g(x, y)=\int_{-\infty}^{\infty} \int_{-\infty} f(\xi, \eta) \operatorname{PSF}(x-\xi, y-\eta) d \xi d \eta
$$

This is the 2D convolution integral, which is often abbreviated as

$$
g=f * * \mathrm{PSF},
$$

where the dual asterisks denote a $2 \mathrm{D}$ convolution. ${ }^{10}$

The derived expressions (Eqs. 4.11 and 4.12) have the following important meaning. If one obtains the PSF of a linear shift-invariant imaging system by imaging a very simple, point object (delta function), then one can calculate the image of an arbitrarily complex object by simply convolving the corresponding input function with the PSF. In this sense, the PSF is a general transfer characteristic of an imaging system in the spatial domain and, hence, contains all the information necessary to predict the response of the system to any particular input. ${ }^{49}$ Accordingly, the PSF represents the most complete spatial-domain measure of spatial resolution. In general, the PSF is asymmetric and has a different shape in different directions. For certain imaging systems, however, the PSF exhibits rotational symmetry. ${ }^{47}$ The analysis of such systems, which are called isotropic, is greatly simplified because their PSF can be written as a 1D function. 
Direct measurement of the PSF is difficult for two experimental reasons. ${ }^{47}$ First, when approximating a point source with a tiny aperture, the size of the aperture must be small compared to the value of spatial resolution being measured. The small aperture leads to a very low intensity of the output x-ray beam and, thus, to a reduced SNR in the acquired data. Second, the measuring procedure inevitably involves determination of the x-ray intensity exactly in the center of the PSF. This may cause alignment difficulties. The described experimental problems can be overcome by first measuring one of the other transfer characteristics of an imaging system and then calculating the PSF.

\subsubsection{Line spread function}

The next descriptor of spatial resolution in the spatial domain includes the line spread function (LSF). The LSF is defined as a pixel intensity distribution in the image of an infinitely narrow and infinitely long slit (line source) of unit intensity. ${ }^{47}$ In an ideal imaging system, this distribution would correspond to a line in the image plane. In practical systems, however, the intensity distribution is smeared out around the ideal line and, therefore, forms a blurred image of the line source. The LSF gives a measure of this blurring.

Formally, the LSF is an image of a function $\ell(\xi)$ representing a unit-intensity line,

$$
\operatorname{LSF}(x)=L\{\ell(\xi)\} .
$$

The "line" function can be regarded as an integral of the 2D delta function over the variable that corresponds to the line direction, ${ }^{7,46}$ 


$$
\ell(\xi)=\int_{-\infty}^{\infty} \delta(\xi, \eta) d \eta
$$

Then, using the linearity property, the LSF is given by

$$
\operatorname{LSF}(x)=\int_{-\infty}^{\infty} L\{\delta(\xi, \eta)\} d \eta
$$

this can be simplified recalling the definition of the PSF (Eq. 4.6):

$$
\operatorname{LSF}(x)=\int_{-\infty}^{\infty} \operatorname{PSF}(x, y) d y
$$

Thus, the LSF is equal to the integral of the PSF.

In the special case of a 1D object, $f(\xi)$, the expression relating the output of an imaging system and its PSF (Eq. 4.11) can be rewritten as

$$
\left.g(x, y)\right|_{y=\text { const }}=\int_{-\infty}^{\infty} f(\xi)\left[\left.\int_{-\infty}^{\infty} \operatorname{PSF}(x-\xi, y-\eta)\right|_{y=\text { const }} d \eta\right] d \xi,
$$

which after employing the formula derived for the LSF (Eq. 4.16) becomes

$$
g(x)=\int_{-\infty}^{\infty} f(\xi) \operatorname{LSF}(x-\xi) d \xi
$$

This is the 1D convolution integral, frequently presented in the short form as

$$
g=f * \mathrm{LSF},
$$

where the single asterisk indicates a 1D convolution.

The obtained expressions (Eqs. 4.18 and 4.19) state that for the 1D case, if one finds the LSF, in a certain direction, of a linear shift-invariant imaging system by imaging a very simple, line object, then one can calculate the image, in the same direction, of an arbitrarily complex object by convolving the corresponding input function with the LSF. Hence, the LSF represents a spatial-domain transfer characteristic of an imaging system 
in the special case of 1D inputs. Consequently, the LSF is a 1D measure of spatial resolution in the spatial domain.

Measurement of the LSF reduces the experimental difficulties associated with the

direct determination of the PSF. ${ }^{46,47}$ However, because the LSF is a 1D representation of the 2D PSF in one particular direction, the former must be measured in all possible directions before the actual PSF can be calculated. This approach is greatly simplified for isotropic imaging systems. In such systems, the PSF is rotationally-symmetric, and the shape of the LSF does not depend on the direction in which the LSF is measured. Thus, for isotropic imaging systems, one measurement of the LSF suffices for the calculation of the PSF. ${ }^{47}$

\subsubsection{Edge spread function}

The final spatial-domain descriptor of spatial resolution is the edge spread function (ESF). By definition, the ESF refers to a distribution of pixel intensities in the image of an infinitely long, perfectly attenuating edge (step source) of unit intensity. In an ideal imaging system, this distribution would correspond to a step in the image plane. In practical systems, however, the intensity distribution is smeared out around the ideal step and, therefore, forms a blurred image of the step source. The ESF offers a measure of this blurring.

The ESF is mathematically an image of a unit step function $h(\xi)$,

$$
\operatorname{ESF}(x)=L\{h(\xi)\}
$$

The unit step function can be written as ${ }^{7,46}$ 


$$
h(\xi)=\int_{-\infty}^{\xi} \ell\left(\xi^{\prime}\right) d \xi^{\prime}
$$

where $\ell\left(\xi^{\prime}\right)$ is the function representing a unit-intensity line (Eq. 4.14). Then, taking into account the linearity property, the ESF is given by

$$
\operatorname{ESF}(x)=\int_{-\infty}^{x} L\left\{\ell\left(\xi^{\prime}\right)\right\} d \xi^{\prime}
$$

which after using the definition of the LSF (Eq. 4.13) becomes

$$
\operatorname{ESF}(x)=\int_{-\infty}^{x} \operatorname{LSF}\left(x^{\prime}\right) d x^{\prime}
$$

Conversely,

$$
\frac{d}{d x} \operatorname{ESF}(x)=\operatorname{LSF}(x)
$$

Hence, the derivative of the ESF produces the LSF, and, therefore, the expressions developed to describe the relationship between the output of an imaging system and its LSF (Eqs. 4.18 and 4.19) can be applied here after the ESF is differentiated.

Similarly to the LSF, the ESF serves as a transfer characteristic of a linear shiftinvariant imaging system in the 1D case and, accordingly, represents a $1 \mathrm{D}$ measure of spatial resolution in the spatial domain. The ESF, however, provides two obvious practical advantages compared with the LSF. First, an edge used to measure the ESF is easier to fabricate than a narrow slit employed in the LSF measurement. ${ }^{50}$ Second, the use of an edge offers much better x-ray tube utilization and, thus, places a much lower load on the x-ray tube than the use of the slit, which causes excessive tube loading due to a very small width needed to approximate a line source. ${ }^{45}$ As in the case of the LSF, the ESF must be measured in all possible directions in order to obtain a complete, 2D 
description of an imaging system. Again, for an isotropic system, one ESF measurement is sufficient for such a description.

\subsubsection{Frequency-domain measures}

In the spatial-frequency domain, spatial resolution of an imaging system is given by such transfer characteristics as the optical transfer function, modulation transfer function, and phase transfer function. These measures specify how efficiently various frequencies in an object are transferred by the system when it forms an image. ${ }^{50}$

\subsubsection{Optical transfer function}

The first frequency-domain descriptor of spatial resolution is the optical transfer function (OTF). Formally, the OTF corresponds to the Fourier transform of the PSF,

$$
\operatorname{OTF}(u, v)=\mathcal{F}\{\operatorname{PSF}(x, y)\}
$$

where $u$ and $v$ are spatial frequencies. This expression assumes that the PSF is defined with a point source of unit intensity (as described in the subsection 4.2.1.1). In practice, however, the point source may have intensity that differs from one. To account for this difference, the practical OTF is usually normalized by the total volume under the PSF: ${ }^{51}$

$$
\operatorname{OTF}^{*}(u, v)=\frac{\operatorname{OTF}(u, v)}{\iint_{-\infty}^{\infty} \operatorname{PSF}(x, y) d x d y}=\frac{\operatorname{OTF}(u, v)}{\operatorname{OTF}(0,0)}
$$

where the second denominator follows from the definition of the OTF (Eq. 4.25). Such normalization ensures that the OTF has a unit value at zero frequencies. Because the current discussion is based on the "proper" definition of the PSF, the former version of the OTF (Eq. 4.25) will be considered. 
The importance of the OTF can be established by examining the special case of a sinusoidal object (i.e., an object whose radiation intensity varies sinusoidally with position in the object plane). Such an object is generally expressed in terms of a complex exponential, ${ }^{49}$

$$
f(\xi, \eta)=e^{i 2 \pi(u \xi+v \eta)} .
$$

The image of this object can be calculated using Eq. 4.11 and the definition of the Fourier transform (Eq. 4.4):

$$
\begin{gathered}
g(x, y)=\iint_{-\infty}^{\infty} f(x-\xi, y-\eta) \operatorname{PSF}(\xi, \eta) d \xi d \eta= \\
=\int_{-\infty}^{\infty} \int^{i 2 \pi[u(x-\xi)+v(y-\eta)]} \operatorname{PSF}(\xi, \eta) d \xi d \eta=\left[\iint_{-\infty}^{\infty} \operatorname{PSF}(\xi, \eta) e^{-i 2 \pi(u \xi+v \eta)} d \xi d \eta\right] \times \\
\times e^{i 2 \pi(u x+v y)}=\mathcal{F}\{\operatorname{PSF}(\xi, \eta)\} e^{i 2 \pi(u x+v y)}=\operatorname{OTF}(u, v) e^{i 2 \pi(u x+v y)} .
\end{gathered}
$$

Thus, combining Eqs. 4.1, 4.27, and 4.28, one obtains

$$
g(x, y)=L\left\{e^{i 2 \pi(u \xi+v \eta)}\right\}=\operatorname{OTF}(u, v) e^{i 2 \pi(u x+v y)},
$$

which states that a sinusoidal object produces a sinusoidal image of the same spatial frequencies but different amplitude and phase; the latter two parameters are scaled by the frequency-dependent factor $\operatorname{OTF}(u, v) \cdot{ }^{49}$ Hence, the OTF describes the transfer of sinusoidal inputs through a linear shift-invariant imaging system. ${ }^{47}$

In the general case, any object can be decomposed into an infinite sum of sinusoids, by employing the inverse Fourier transform (Eq. 4.5),

$$
f(\xi, \eta)=\mathcal{F}^{-1}\{F(u, v)\}=\int_{-\infty}^{\infty} \int_{-\infty} F(u, v) e^{i 2 \pi(u \xi+v \eta)} d u d v,
$$

Again, following Eq. 4.11, one can find the image of this arbitrary object: 


$$
\begin{gathered}
g(x, y)=\int_{-\infty}^{\infty} \int\left[\int_{-\infty}^{\infty} \int_{-\infty}^{\infty} F(u, v) e^{i 2 \pi[u(x-\xi)+v(y-\eta)]} d u d v\right] \operatorname{PSF}(\xi, \eta) d \xi d \eta= \\
=\int_{-\infty}^{\infty} \int^{\infty} F(u, v)\left[\int_{-\infty}^{\infty} \int_{-\infty} \operatorname{PSF}(\xi, \eta) e^{-i 2 \pi(u \xi+v \eta)} d \xi d \eta\right] e^{i 2 \pi(u x+v y)} d u d v= \\
=\int_{-\infty}^{\infty} \int^{\infty} F(u, v) \operatorname{OTF}(u, v) e^{i 2 \pi(u x+v y)} d u d v
\end{gathered}
$$

The left side of this equation can also be expressed as the inverse Fourier transform, ${ }^{49}$

$$
g(x, y)=\mathcal{F}^{-1}\{G(u, v)\}=\int_{-\infty}^{\infty} \int_{-\infty} G(u, v) e^{i 2 \pi(u x+v y)} d u d v
$$

By comparing the last two equations, one gets

$$
G(u, v)=F(u, v) \operatorname{OTF}(u, v),
$$

where $F(u, v)$ is the Fourier transform of the object $f(\xi, \eta)$, and $G(u, v)$ is the Fourier transform of the image $g(x, y)$.

The derived expression (Eq. 4.33) is a frequency-domain analog of the convolution formula (Eq. 4.11 or 4.12). This expression shows that the OTF is a frequency-dependent scaling factor for every sinusoid in the Fourier representation of an arbitrary object. Thus, the OTF describes the transfer of any, not just sinusoidal, input through a linear shift-invariant imaging system. ${ }^{47}$ Therefore, the OTF is the general transfer characteristic of an imaging system in the frequency domain, similarly to the PSF in the spatial domain. Consequently, the OTF is the most complete frequency-domain measure of spatial resolution. In general, the OTF is complex and asymmetric. For an isotropic imaging system, however, the OTF becomes real and rotationally-symmetric; it can be then calculated as the Hankel transform of the PSF. ${ }^{10,48}$

The final equation (Eq. 4.33) has another important meaning. According to this 
equation, the mathematically complicated convolution in the spatial domain is replaced by simple multiplication in the frequency domain. ${ }^{47}$ Such replacement greatly facilitates the analysis of imaging systems and is especially useful for the study of cascaded (or serial) systems. ${ }^{48}$ These systems consist of several components connected in series, so that the output from one component is the input to the next component. ${ }^{47}$ The overall OTF of a cascaded imaging system can be easily calculated by multiplying the OTFs of all the components, rather than convolving their PSFs. Many practical imaging systems can be viewed as cascaded systems, to allow a detailed analysis of their performance, as will be demonstrated later in this chapter.

Although the OTF is a 2D function in general, its 1D "profiles" along directions that pass through the origin of the $(u, v)$ plane are often used in various studies. It can be shown that each of these profiles is given by the 1D Fourier transform of the corresponding LSF or the differentiated ESF. ${ }^{48}$ Specifically, for the profile along the $u$ axis,

$$
\operatorname{OTF}(u, 0)=\mathcal{F}\{\operatorname{LSF}(x)\}=\mathcal{F}\left\{\frac{d}{d x} \operatorname{ESF}(x)\right\} .
$$

Hence, the 2D OTF can be experimentally determined from the 1D Fourier transforms of the LSFs measured in all possible directions. For an isotropic imaging system, one such a transform of one LSF provides all the 2D information.

\subsubsection{Modulation transfer function}

The next frequency-domain descriptor of spatial resolution involves the modulation transfer function (MTF). In mathematical terms, the MTF is simply the modulus of the OTF, 


$$
\operatorname{MTF}(u, v)=|\operatorname{OTF}(u, v)|
$$

This equation assumes that the OTF is properly-normalized (as discussed in the previous subsection), so that the MTF has a unit value at zero frequencies. If the OTF is not normalized, then the omitted normalization factor should be included in the MTF: ${ }^{49}$

$$
\operatorname{MTF}^{*}(u, v)=\frac{\operatorname{MTF}(u, v)}{\iint_{-\infty}^{\infty} \operatorname{PSF}(x, y) d x d y}=\frac{\operatorname{MTF}(u, v)}{\operatorname{OTF}(0,0)}=\frac{\operatorname{MTF}(u, v)}{\operatorname{MTF}(0,0)}
$$

(Here, the modulus in the first and second denominators is not needed because the total volume under the PSF is always positive.) The current discussion is based on the assumption of the properly-normalized OTF; therefore, the first expression for the MTF (Eq. 4.35) will be employed.

To explain the significance of the MTF, it is convenient to consider a sinusoidal object given by a scaled and raised complex exponential: ${ }^{49}$

$$
f(\xi, \eta)=a+b e^{i 2 \pi(u \xi+v \eta)},
$$

where $a$ and $b$ are real non-negative numbers. For this object, one can define a parameter called modulation, ${ }^{49,52}$

$$
M_{f}=\frac{\left|f_{\text {max }}\right|-\left|f_{\text {min }}\right|}{\left|f_{\text {max }}\right|+\left|f_{\text {min }}\right|}=\frac{(a+b)-(a-b)}{(a+b)+(a-b)}=\frac{b}{a},
$$

where $f_{\max }$ and $f_{\min }$ are the maximum and minimum values, respectively, of the function $f(\xi, \eta)$. The image of such an object can be found utilizing the linearity property of an imaging system (Eq. 4.2), the expression for the system response to a sinusoidal input (Eq. 4.29), and the assumption that $\operatorname{OTF}(0,0)=1$ : 


$$
\begin{gathered}
g(x, y)=L\left\{a+b e^{i 2 \pi(u \xi+v \eta)}\right\}=L\{a\}+L\left\{b e^{i 2 \pi(u \xi+v \eta)}\right\}= \\
=a L\left\{\left.e^{i 2 \pi(u \xi+v \eta)}\right|_{u=0, v=0}\right\}+b L\left\{e^{i 2 \pi(u \xi+v \eta)}\right\}= \\
=a \operatorname{OTF}(0,0)+b \operatorname{OTF}(u, v) e^{i 2 \pi(u \xi+v \eta)}= \\
=a+b \operatorname{OTF}(u, v) e^{i 2 \pi(u \xi+v \eta)} .
\end{gathered}
$$

Modulation for the image can be then calculated as

$$
\begin{gathered}
M_{g}=\frac{\left|g_{\text {max }}\right|-\left|g_{\text {min }}\right|}{\left|g_{\text {max }}\right|+\left|g_{\text {min }}\right|}=\frac{|a+b \operatorname{OTF}(u, v)|-|a-b \operatorname{OTF}(u, v)|}{|a+b \operatorname{OTF}(u, v)|+|a-b \operatorname{OTF}(u, v)|}= \\
=\frac{b}{a}|\operatorname{OTF}(u, v)|=M_{f}|\operatorname{OTF}(u, v)| .
\end{gathered}
$$

From Eqs. 4.40 and 4.35, the MTF is thus the ratio of image modulation to object modulation, expressed as a function of spatial frequencies,

$$
\operatorname{MTF}(u, v)=\frac{M_{g}}{M_{f}}
$$

In other words, the MTF represents a frequency-dependent scaling factor for modulation, or amplitude, of a sinusoidal input when the latter is transferred through a linear shiftinvariant imaging system. Due to the fact that the ratio $M_{g} / M_{f}$ by itself is known as the modulation transfer, the functional form of this ratio was named the "modulation transfer function." 52,53

Because any object can be specified in the frequency domain as a sum of an infinite number of sinusoids, and the MTF scales the amplitudes of sinusoids, the MTF, in general, describes the transfer of the Fourier amplitudes of an arbitrary, not just sinusoidal, input through a linear shift-invariant imaging system. This is demonstrated by taking the absolute values of the functions in Eq. 4.33:

$$
|G(u, v)|=|F(u, v)| \operatorname{MTF}(u, v)
$$

The Fourier amplitudes expressed as a function of spatial frequencies are also called the 
amplitude spectrum. Hence, the MTF is the frequency-domain transfer characteristic of an imaging system in terms of the amplitude spectrum. Due to the disposal of phase information, the MTF is not as complete a system descriptor as the OTF. ${ }^{49}$ However, for most imaging systems, the effects of phase on the image quality are believed to be negligible, and the MTF is commonly accepted as an accurate measure of spatial resolution in the frequency domain. ${ }^{54}$ The MTF is always real and non-negative; the 1D MTF is also symmetric, because of the real PSF of practical x-ray imaging systems. For isotropic systems, the MTF is equivalent to the OTF, except for the sign, and, therefore, virtually no phase information is lost with the use of the MTF. ${ }^{7,49}$

Similarly to the OTF, the MTF is normally viewed as a $2 \mathrm{D}$ function. In many studies, however, only 1D "profiles" of this function along certain directions that pass through the origin of the spatial-frequency plane are considered. These profiles, or 1D MTFs, can be calculated, following Eq. 4.34, as

$$
\operatorname{MTF}(u)=|\mathcal{F}\{\operatorname{LSF}(x)\}|=\left|\mathcal{F}\left\{\frac{d}{d x} \operatorname{ESF}(x)\right\}\right| .
$$

Thus, the experimental evaluation of the 1D MTF is reduced to the measurement of the appropriate LSF or ESF. To obtain the 2D MTF, such a measurement must be repeated in all possible directions, unless an imaging system is isotropic, in which case one LSF or ESF measurement gives all the necessary information.

\subsubsection{Phase transfer function}

The last frequency-domain descriptor of spatial resolution is the phase transfer function (PTF). According to the formal expression, the PTF represents the phase portion of the OTF, 


$$
\operatorname{PTF}(u, v)=\phi_{\text {OTF }}(u, v)
$$

The PTF is not normalized, and neither does it require the OTF to be normalized (the normalization factor cancels out when the PTF is calculated).

Utilizing the approach similar to one in the MTF explanation, it can be shown that the PTF is a frequency-dependent scaling factor for the phase of a sinusoidal object that is being imaged by a linear shift-invariant imaging system. Furthermore, it can be generalized, on the basis of the Fourier representation of an arbitrary object, that the PTF describes the transfer of all the phases (i.e., the phase spectrum) in the case of any, not just sinusoidal, object. Therefore, the PTF is the transfer characteristic of an imaging system in terms of the phase spectrum in the frequency domain. Although the PTF is, in principle, a frequency-domain measure of spatial resolution, this function is almost never used alone, due to insufficient information it provides. Rather, the PTF serves as an adjunct to the MTF. Together, the PTF and MTF represent a different way of expressing the complex OTF - in terms of its modulus and phase:

$$
\operatorname{OTF}(u, v)=|\operatorname{OTF}(u, v)| e^{i \phi_{\text {OTF }}(u, v)}=\operatorname{MTF}(u, v) e^{i \operatorname{PTF}(u, v)} .
$$

For an isotropic imaging system, the PSF is rotationally-symmetric, and the PTF assumes its simplest form with values of either zero or $\pi \cdot{ }^{53}$ Because, in this case, the spatial frequencies at which the PTF switches between these two values are usually fairly evident from the $\mathrm{MTF}^{7}$ the PTF does not provide much new information and can be safely omitted. The situation differs, however, for an imaging system with the asymmetric PSF. The latter condition generally results in the nonlinear PTF, leading to so-called phase distortions, when different spatial frequencies recombine in the image with different relative phases. ${ }^{53}$ This degrades the diagnostic image quality, but such 
degradation is not fully described by the MTF. ${ }^{54}$ Consequently, the PTF cannot be discarded and should always complement the MTF when studying an imaging system with the potentially-asymmetric PSF.

\subsubsection{Summary of measures}

As discussed in this section, the three "spread" functions in the spatial domain and the three "transfer" functions in the frequency domain can be used to quantify spatial resolution of an imaging system. Among these functions, the spatial-domain PSF and its frequency-domain analog, the OTF, provide the full system description and, accordingly, represent the complete measures of spatial resolution. Despite their completeness, the 2D PSF and OTF are rarely evaluated as part of routine resolution assessment, mainly because such evaluation requires a large number of measurements in the general case of an anisotropic system. Therefore, instead of the PSF and OTF, practical imaging systems are often characterized by the LSF (or ESF) and 1D MTF. The latter functions are typically measured in two orthogonal directions ( $x$ and $y$ or radial and azimuthal) for anisotropic 2D systems and only in one direction for isotropic or 1D systems. Overall, the 1D MTF is recognized by the engineering and scientific community as the most convenient measure of spatial resolution, ${ }^{45}$ and the LSF and ESF are viewed as experimental tools for determining this MTF. In the further discussion, the 1D MTF will be referred to as simply the MTF.

When the MTF is employed, the information about spatial resolution is contained in the shape of this function. An imaging system with a higher MTF curve would generally have better resolution. In addition to the shape, several MTF-based single- 
number specifications of spatial resolution are also important. The most common is the cutoff frequency (in cy/mm), which corresponds to the spatial frequency at which the MTF reaches its first zero. An imaging system with a higher MTF curve and a higher cutoff frequency would definitely have better resolution. Often, the MTF approaches but never reaches zero; in this case, the frequency at which the MTF falls below a certain threshold (e.g., 0.05 or 0.01 ) is used as the cutoff frequency. The threshold is typically chosen from the condition of having no detectable or visible information in the image below the threshold value. Another single-number descriptor of spatial resolution is the area under the MTF curve in the range from zero to the cutoff frequency. ${ }^{53}$ This area indicates the overall image-quality performance of an imaging system and is helpful when comparing different systems. Although the single-number specifications are more convenient to use than the function, only the whole MTF, via its shape, provides all the available information about spatial resolution.

Similarly, one can assess resolution properties of an imaging system by examining the shape of the LSF, although this practice is less common than studying the MTF shape. Also, for the LSF, a useful single-number descriptor of spatial resolution is the FWHM (in mm). Alternatively, the FWTM or the full width at some other fraction of the LSF maximum can be utilized as well.

It should be noted that neither the LSF nor the 1D MTF serves as a magical shortcut to reduce the $2 \mathrm{D}$ imaging problem to a $1 \mathrm{D}$ task. ${ }^{47}$ To calculate the image of a $2 \mathrm{D}$ object, the PSF, OTF, or 2D MTF is still required, and the LSF (or ESF) is just an accurate experimental tool to help determine those functions. The LSF or 1D MTF can simplify the overall problem only in the case of $1 \mathrm{D}$ objects, but even then, the $2 \mathrm{D}$ nature 
of an imaging system must be taken into account when the system is anisotropic. ${ }^{47}$ Hence, the LSF and 1D MTF are just handy, not difficult to evaluate, and easy to interpret measures of spatial resolution, but they cannot replace the complete system description that would be given by the PSF or OTF.

\subsection{Components of spatial resolution}

For an in-depth analysis of a practical imaging system, it is convenient to view its image-formation process (imaging chain) as a series of several components rather than consider the whole system as a single "black box." Each component is then described in terms of its own input-output relationship, and the serial combination of such relationships for all the components gives the overall system response. This cascadedsystem approach is especially useful for the analysis of spatial resolution because it enables expression of the resolution properties of the entire imaging system in terms of resolution properties of the individual components. Each component adds some blurring to the final image; any such blurring can be treated in the frequency domain as an OTF or

MTF as long as the blur is constant over the whole image. ${ }^{51}$ Thus, each component can be thought as having its own spatial resolution, represented by the OTF or MTF. The overall resolution of the imaging system is then given by the product of the individual OTFs, as described in the previous section, or, in a similar way, by the product of the corresponding MTFs.

In the simplest case of an analog imaging system (Fig. 4.1-a), its imaging chain consists of two basic components: $\mathrm{x}$-ray image formation and $\mathrm{x}$-ray detection. The first component, x-ray image formation, includes the effect of geometric unsharpness, which 


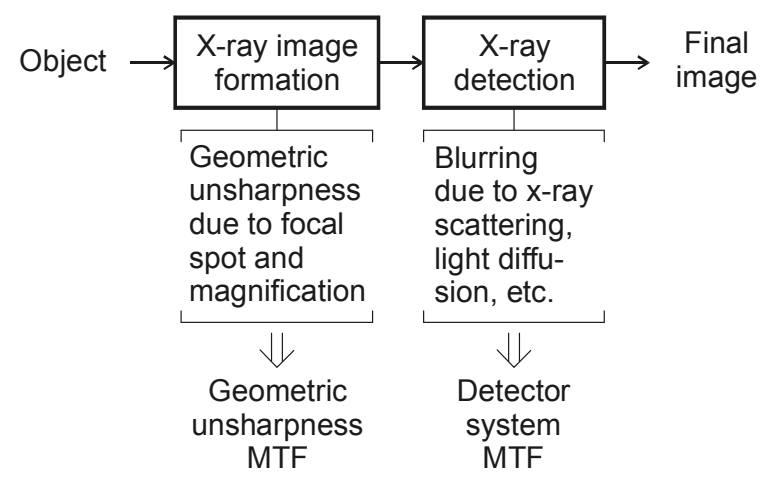

(a)

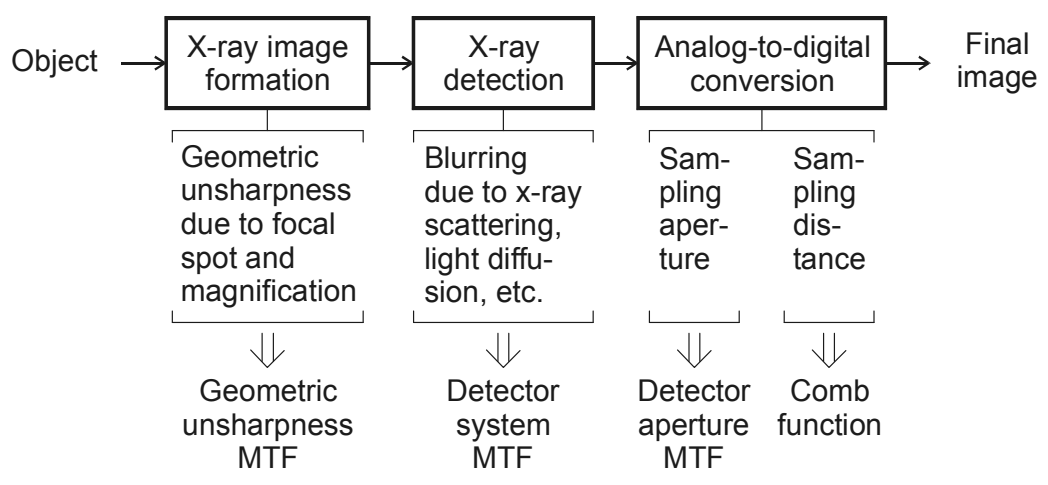

(b)

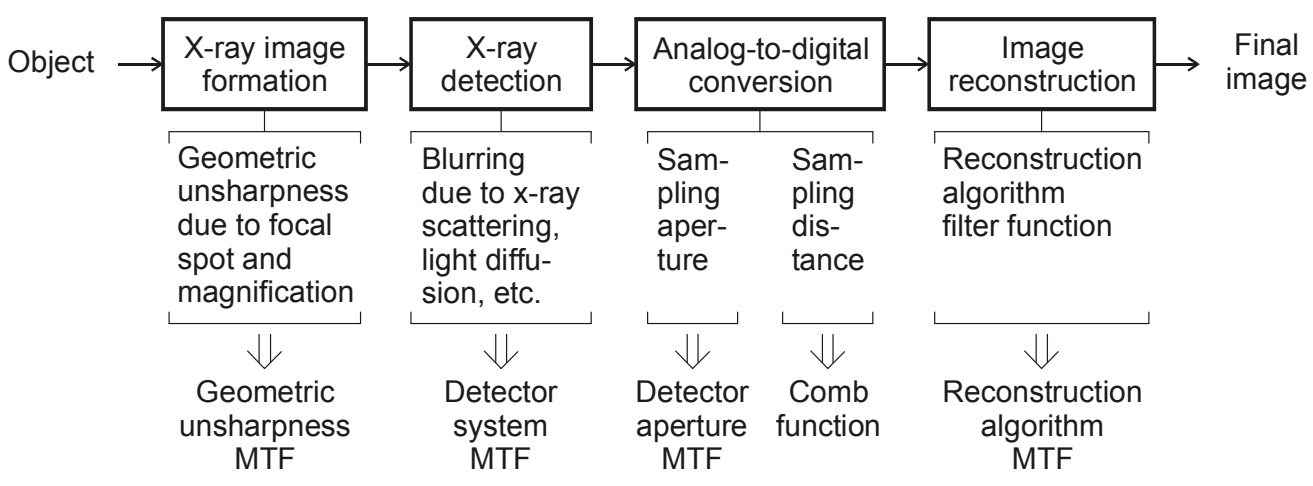

(c)

Figure 4.1. Components contributing to spatial resolution in various imaging systems: (a) analog system, (b) digital system, and (c) CT scanner. 
refers to the loss in image detail caused by the finite size of the x-ray tube focal spot. ${ }^{46,55}$ In addition to the size of the focal spot, geometric unsharpness is also influenced by system magnification, determined by the location of an object between the x-ray tube and the detector. Both these factors - the focal-spot size and system magnification - give rise to the geometric unsharpness $\operatorname{MTF}, \operatorname{MTF}_{\mathrm{GU}}(u)$. The second component, $\mathrm{x}$-ray detection, involves an additional broadening of the system response due to x-ray scattering, light diffusion, and electron spreading in the detection medium. ${ }^{55}$ This broadening is specified by the detector system MTF, $\operatorname{MTF}_{\mathrm{DS}}(u)$. The described imaging chain does not include such a typical component as image display. Although this component is an integral part of an analog, as well as any other, imaging system, image display does not currently represent a serious limitation to system spatial resolution, and therefore will not be discussed in this section. ${ }^{50}$ Hence, the overall MTF of an analog imaging system - the socalled "analog" MTF - is given by

$$
\operatorname{MTF}_{\mathrm{A}}(u)=\operatorname{MTF}_{\mathrm{GU}}(u) \operatorname{MTF}_{\mathrm{DS}}(u) .
$$

A digital imaging system (Fig. 4.1-b) has the same first two components as the analog system. After x-ray detection, however, the acquired image in the digital system is converted from the analog to the digital form. Consequently, the imaging chain of a digital imaging system includes an extra component responsible for the analog-to-digital conversion. Such conversion introduces the effects of sampling aperture, sampling distance, and quantization of the system response. ${ }^{55}$ The sampling aperture serves as a means of local averaging and is usually defined by the size and shape of the detector cells. The effect of sampling aperture is specified by the detector aperture MTF, $\operatorname{MTF}_{\mathrm{DA}}(u)$. The sampling distance indicates the spatial interval at which the image is 
sampled. Assuming the sampling distance in the $x$ direction is $\Delta x$, the process of sampling can be described as convolution of the before-sampling (presampling) MTF with the following comb function: ${ }^{49,55}$

$$
\operatorname{III}(u ; \Delta x)=\sum_{n=-\infty}^{\infty} \delta\left(u-\frac{n}{\Delta x}\right),
$$

where $\delta(u)$ is a $1 \mathrm{D}$ delta function. The effect of the last factor in the analog-to-digital conversion, quantization of the system response, is typically negligible due to a sufficient number of quantization levels available in most practical imaging systems; this effect is often ignored. ${ }^{55,56}$ Thus, the expression for the overall MTF of a digital imaging system the "digital" MTF - can be written as

$$
\begin{aligned}
& \operatorname{MTF}_{\mathrm{D}}(u)=\left[\operatorname{MTF}_{\mathrm{A}}(u) \operatorname{MTF}_{\mathrm{DA}}(u)\right] * \operatorname{III}(u ; \Delta x)= \\
& =\left[\operatorname{MTF}_{\mathrm{GU}}(u) \operatorname{MTF}_{\mathrm{DS}}(u) \operatorname{MTF}_{\mathrm{DA}}(u)\right] * \operatorname{III}(u ; \Delta x) .
\end{aligned}
$$

Finally, in the case of a CT scanner (Fig. 4.1-c), its imaging chain can be viewed as constructed from the imaging chain of the digital imaging system by adding a component that represents image reconstruction. Although the effect of this component on the scanner resolution is not at all straightforward, image reconstruction, in simple terms, corresponds to filtering of the system response with a filter function determined by the reconstruction algorithm. ${ }^{50}$ Mathematically, this function is given by the reconstruction algorithm $\operatorname{MTF}, \operatorname{MTF}_{\mathrm{RA}}(u)$. The imaging chain of a practical CT scanner also includes several data-correction and image-processing components. For simplicity, those components are not considered here, but, if needed, their effects can be characterized similarly, using proper filter functions ${ }^{55,57}$ For the described CT scanner, the overall MTF - or, in other words, the "reconstruction" MTF - is then calculated as 


$$
\begin{aligned}
& \operatorname{MTF}_{\mathrm{R}}(u)=\operatorname{MTF}_{\mathrm{D}}(u) \operatorname{MTF}_{\mathrm{RA}}(u)=\left\{\left[\operatorname{MTF}_{\mathrm{GU}}(u) \times\right.\right. \\
& \left.\left.\times \operatorname{MTF}_{\mathrm{DS}}(u) \operatorname{MTF}_{\mathrm{DA}}(u)\right] * \operatorname{III}(u ; \Delta x)\right\} \operatorname{MTF}_{\mathrm{RA}}(u) .
\end{aligned}
$$

The MTFs of various components of the analog, digital, and CT imaging systems can be summarized as follows (Fig. 4.2). The geometric unsharpness MTF is multiplied by the detector system MTF to produce the analog MTF (Eq. 4.46). The product of the analog MTF and the detector aperture MTF gives the system presampling MTF. This MTF describes the response of an imaging system up to, but not including, the process of sampling. ${ }^{45}$ Although the system presampling MTF is a valuable measure of spatial resolution, it is often desirable to exclude from this MTF the effect of geometric unsharpness, in order to analyze the resolution properties of the detector itself, without influence of the focal spot and magnification. This can be achieved by considering the detector presampling MTF, which is just the product of the detector system MTF and the detector aperture MTF. In the literature, the detector presampling MTF is frequently referred to as simply the "presampling MTF," but in the current discussion, the terms "detector" and "system" will be used to distinguish between the two types of the presampling MTF. Further along the imaging chain, the system presampling MTF is convolved with the comb function to form the digital MTF (Eq. 4.48). At last, the multiplication of the digital MTF with the reconstruction algorithm MTF results in the reconstruction MTF (Eq. 4.49). Any additional MTFs (e.g., for image processing, image display, etc.), if taken into account, would be included in the resolution analysis as multipliers of the already mentioned MTFs.

Although seemingly the same measure - the MTF - is employed to describe spatial resolution of the analog and digital imaging systems, several important differences exist between the analog MTF and the digital MTF. The analog MTF uniquely maps the 


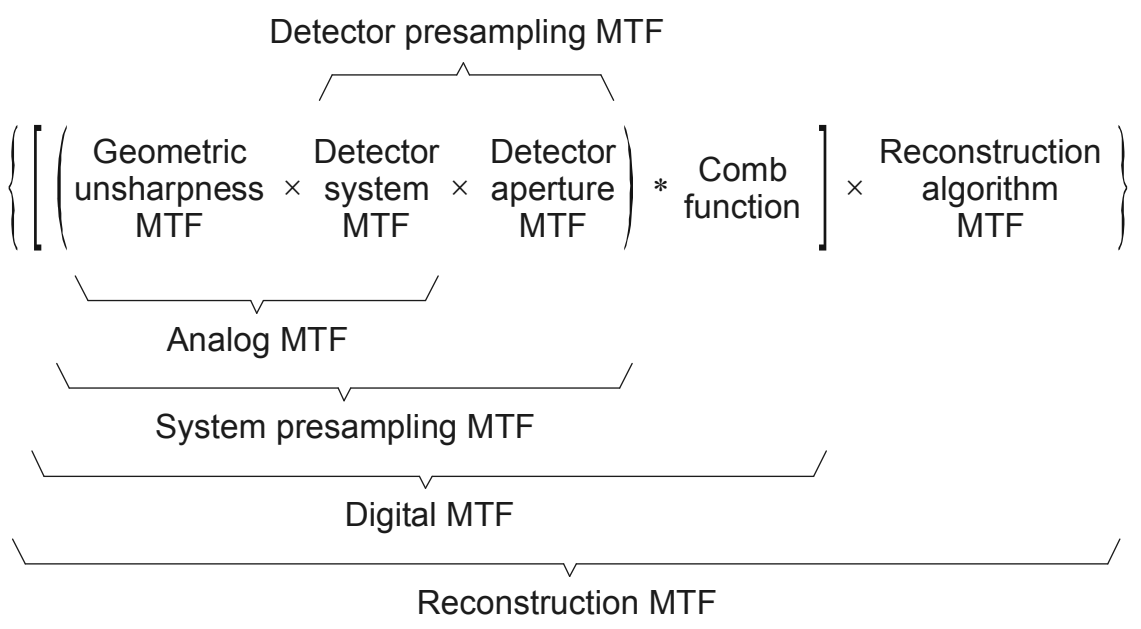

Figure 4.2. MTF hierarchy. 
amplitudes of input frequency components to the amplitudes of output frequency components. ${ }^{57}$ Accordingly, the analog MTF allows a reliable quantitative comparison of two or more analog systems. This is not generally true for the digital MTF. In a digital imaging system, the process of sampling causes replication of the Fourier transform in the frequency domain. ${ }^{57,58}$ If the system is sufficiently-sampled (i.e., its Nyquist frequency equals or exceeds the highest spatial frequency in the before-sampling image of an object being examined), the resulting replicas do not overlap, and the digital MTF has the same properties as the analog MTF. In practice, however, digital imaging systems are almost always undersampled due to design constraints. ${ }^{53,57}$ Undersampling leads to overlapping of the Fourier-transform replicas and, as a result, to aliasing, when frequencies above the Nyquist frequency "fold over" and contaminate their counterparts below the Nyquist frequency. ${ }^{55-57}$ Aliasing, in turn, has two consequences that alter the properties of the digital MTF and complicate its interpretation. ${ }^{58}$ First, the digital MTF contains a "false" (not representing higher spatial resolution) increase at frequencies where the replicas overlap. ${ }^{55}$ Hence, the digital MTF no longer uniquely describes the transfer of the Fourier amplitudes and, therefore, cannot be used for a quantitative comparison of digital systems. Second, the digital MTF is phase-dependent, i.e., it has different values for different positions of the sampling comb function with respect to the system presampling MTF. Phase-dependence indicates that, in the presence of aliasing, a digital imaging system is no longer shift-invariant, as required by the transfer-theory approach. This problem can be overcome by averaging the digital MTFs over all possible phases; the resulting function is called the expectation MTF. ${ }^{45,57}$ Although the expectation MTF solves the phase-dependence problem, this function is still not a unique 
descriptor of digital-system spatial resolution - the expectation MTF still includes the object-dependent aliasing artifacts, which may cause two systems with identical resolution properties to have different expectation MTFs ${ }^{58}$ Apparently, for an undersampled digital imaging system affected by aliasing, there is no measure of overall spatial resolution that would uniquely, independently of the object, map the input of the system to its output. ${ }^{45,57,59}$

Because the digital MTF, unlike the analog MTF, does not allow an exact comparison of imaging systems, it has been recommended to include both the digital MTF (in the form of the expectation MTF) and the detector presampling MTF when reporting the resolution properties of a digital imaging system. ${ }^{45,57-59}$ The detector presampling MTF not only avoids all the problems of the digital MTF, but also excludes the effects of the focal spot and magnification; such a feature makes this MTF an ideal tool for comparing inherent spatial resolution of discrete detectors. Due to the limitations of the digital MTF, one would expect similar problems with the reconstruction MTF, in the case of CT scanners. Indeed, the reconstruction MTF gives no unique description of the resolution properties either. Therefore, as with digital systems, the reconstruction MTF should be reported together with the detector presampling MTF when specifying spatial resolution of practical CT scanners. ${ }^{2,50}$ In summary, the analog MTF, the detector presampling MTF, and the reconstruction MTF are usually employed to characterize the resolution properties of the analog, digital, and CT imaging systems, respectively. 


\subsection{Measurement of spatial resolution}

Various methods have been developed to measure spatial resolution of $\mathrm{x}$-ray imaging systems. As already mentioned in Sections 4.2.2.2 and 4.2.3, those methods generally consist in experimental evaluation of the LSF or ESF, in either one or two directions, and then calculation of the corresponding 1D MTF (using Eq. 4.43, for example). Such indirect assessment is much more prevalent than measuring the MTF directly, using phantoms with sinusoidal x-ray transmission, because these phantoms are difficult to fabricate, especially at high spatial frequencies. Due to the fact that, according to the previous section, the three main types of imaging systems - analog, digital, and CT - utilize different definitions of spatial resolution, the measuring procedures vary among the system types. Therefore, the three major groups of methods - to determine the analog MTF, the detector presampling MTF, and the reconstruction MTF - can be considered.

\subsubsection{Analog MTF}

Spatial resolution of analog imaging systems (which are mostly screen-film

combinations) can be measured by either the slit, wire, edge, or bar-pattern method. ${ }^{45,60}$ The first three techniques evaluate the analog MTF via the LSF and ESF, whereas the last technique determines the resolution properties of a system via its square-wave response.

The slit method is based on the definition of the LSF (Section 4.2.1.2). The ideal, infinitely narrow and infinitely long slit with infinitely thin but completely opaque to $\mathrm{x}$ rays jaws is approximated by a practical slit that has a width of about $10 \mu \mathrm{m}$, a finite dimension, and 1-2 mm thick jaws typically made of tungsten, platinum, or lead. ${ }^{46,61,62}$ The slit is usually placed directly onto a screen-film combination, perpendicularly to the 
direction in which the MTF needs to be measured. After an x-ray exposure, the developed film is scanned with a microdensitometer, which passes through the slit image in the direction of the MTF measurement. The acquired data are then linearized, by converting the film density to the relative exposure using the characteristic (H\&D) curve of the film. The linearized data correspond to the measured LSF. Several such LSFs, at various positions along the slit length, are normally obtained and averaged. After averaging, the LSF is converted to the measured MTF by means of the Fourier transform. In order to correct for the finite slit width, the measured MTF is divided by a properly-scaled sinc function representing a rectangular slit. The final result is the analog MTF.

Several experimental factors affect accuracy of the slit method. The first is the width of the slit, which must be narrow enough to avoid significant MTF degradation but wide enough to ensure an adequate SNR in the acquired image. ${ }^{61}$ As a rule of thumb, the slit width, when projected on the image plane, should be around one fifth or less than the expected FWHM of the analog LSF being measured. Another important factor is the length of the LSF curve. Theoretically, the LSF has an infinite spatial extent, but for obvious practical reasons, only a limited length of this function is measured. Thus, the tails of the experimental LSF are almost always truncated, which leads to erroneous oscillations in the corresponding MTF. It has been shown that the truncation error for screen-film combinations becomes negligible when the LSF is measured down to less than $1 \%$ of its peak value. ${ }^{63}$ If there are not enough experimental data to satisfy this requirement, the LSF tails should be extrapolated with exponential or other functions. Extrapolation, fitting, or smoothing of the LSF tails also reduces noise and improves accuracy of the MTF, but one must be careful not to change significantly the shape of the 
tails. $^{60,61}$ An additional factor that influences quality of the slit measurement is the LSF sampling distance. Even for analog imaging systems, the LSF must be sampled before the MTF can be digitally computed. Such sampling is typically done during the scanning of the slit image with a microdensitometer. A too large LSF sampling distance may cause aliasing and, hence, inaccuracies in the final MTF. As demonstrated for screen-film combinations, the aliasing error can be reduced to a negligible level by choosing the sampling distance that is less than about one eighth of the LSF FWHM. ${ }^{64}$

The wire method to measure the analog MTF has many similarities with the slit technique, except for the inverted radiation intensity distribution resulting from the use of a thin tungsten wire instead of a narrow slit. The similar practical considerations - an appropriate width of the wire, a sufficient length of the LSF curve, and a small enough LSF sampling distance - apply to the wire method as well. As an advantage over the slit technique, the wire method has almost no sensitivity to alignment errors. The wire image, however, exhibits higher quantum noise, due to a lower SNR at the peak of the LSF compared with an SNR in the tail region. ${ }^{33}$ For this reason, the wire method is mainly employed for the MTF measurement in low-noise conditions.

The edge method utilizes the idea of the ESF (Section 4.2.1.3). A sharp, highlypolished tungsten or lead edge is placed in front of a screen-film combination to obtain a necessary image during an x-ray exposure. Similarly to the slit method, this image is scanned with a microdensitometer, in the direction perpendicular to the edge, and the acquired data are linearized to form the measured ESF. Several such ESFs, from the same edge image, are averaged, and the result is differentiated to produce the measured LSF (Eq. 4.43). The differentiation can be done either analytically, by first fitting a known 
function to the ESF data, or numerically, by using a finite-element approach. In the former case, the analog MTF is computed directly from the measured LSF. In the case of the finite-element differentiation, an additional MTF correction is required to compensate for a nonlinear frequency response of the numeric approach. ${ }^{65}$ As with the previous techniques, accuracy of the edge method is affected by the ESF sampling distance, which, therefore, should be chosen accordingly. The advantages of the edge method include the already mentioned easier fabrication of an edge and much lower x-ray tube loading (Section 4.2.1.3) as well as more reliable measurement of the MTF low-frequency components. ${ }^{45}$ The main disadvantages are a noisy LSF estimate, due to the ESF differentiation, and difficulties with aligning the edge with respect to the x-ray beam.

A theoretical comparison of the slit, wire, and edge methods has shown that for an analog imaging system whose performance is limited by quantum noise, the MTF measured by the slit method has the maximum SNR, among the three techniques, at high spatial frequencies. ${ }^{60}$ The MTF assessed by the edge method exhibits the highest SNR at low frequencies, and the MTF obtained by the wire method has an SNR that is always lower than that of the slit-technique MTF. Thus, for quantum-noise-limited systems, the slit method is superior for measuring the high-frequency response, the edge method is superior for determining the response at low frequencies, and the wire method is always inferior to the slit technique.

The bar-pattern method (also known as the square-wave technique) offers an alternative approach to evaluating spatial resolution of analog imaging systems. The method employs a bar pattern - a simple phantom consisting of alternating lead bars and air gaps of the progressively narrower width. The bar pattern provides square-wave x-ray 
transmission at several discrete spatial frequencies. During the measurement, the bar pattern is imaged, the film is scanned, and the average maximum and minimum densities at each of the discrete frequencies are determined. ${ }^{62}$ These densities are then linearized and used to compute the corresponding values of the contrast transfer function (CTF). The CTF is defined as frequency-dependent image modulation (Section 4.2.2.2) for a square-wave object. ${ }^{53}$ Because the CTF represents the square-wave response of an imaging system and not the response to a sinusoid, this function is not the same as the MTF. ${ }^{45}$ The CTF, however, can be converted to the MTF by utilizing Coltman's formula: ${ }^{45,53,61,62}$

$$
\operatorname{MTF}(u)=\frac{\pi}{4}\left[\operatorname{CTF}(u)+\frac{1}{3} \operatorname{CTF}(3 u)-\frac{1}{5} \operatorname{CTF}(5 u)+\ldots\right],
$$

where the values of the $\mathrm{CTF}$ at the required spatial frequencies are interpolated from the values measured at the frequencies included in the bar pattern. To emphasize the difference between the bar-pattern spatial resolution measurement expressed via the CTF and the resolution measurement given by the MTF, the former quantity is specified in $\mathrm{lp} / \mathrm{mm}$, instead of cy/mm.

The bar-pattern method, due to its simplicity, provides a quick and convenient way to asses the analog MTF at discrete spatial frequencies. The method is also very useful for estimating the "cutoff frequency" as the frequency at which individual bars are no longer seen. Nevertheless, the bar-pattern method has several precision-related drawbacks. ${ }^{45}$ First, because of noise, it is difficult to accurately determine the maximum and minimum film densities in the bar-pattern image. Second, a small number of frequencies included in a typical commercially-available bar pattern leads to a rather coarse estimate of the frequency response. Third, practical limitations on the number of 
terms in Eq. 4.50 introduce an error during the CTF-to-MTF conversion. Fourth, experimental difficulties with reliable evaluation of the zero-frequency component, which is used as a normalization factor, may cause overestimation of the MTF. ${ }^{66}$ For these reasons, the bar-pattern method is not recommended for accurate measurement of the analog MTF. ${ }^{45}$

\subsubsection{Detector presampling MTF}

In the case of digital imaging systems, the methods to measure the detector presampling MTF can be divided into two sub-groups, depending on the dimensionality of the utilized discrete detectors. For systems with 1D detectors (e.g., linear scintillatorphotodiode arrays), the detector presampling MTF is measured by either the moving-slit or moving-edge method. For systems employing 2D detectors (e.g., flat-panel arrays), the primary methods are with a stationary slanted slit and a stationary slanted edge.

The moving-slit method is identical to the slit technique used for analog MTF evaluation (Section 4.4.1) except the slit, instead of being stationary during an x-ray exposure, is moved along a 1D discrete detector, across a cell under study, while the system response, instead of being read from multiple spatial positions, is recorded from the same cell at many successive time points. Each time point corresponds to a slightly different position of the slit with respect to the cell under study. This way, the movement of the slit provides sufficiently-fine spatial sampling required for measurement of the detector presampling MTF in 1D discrete detectors. Otherwise, if the slit were stationary, and the system response were read from successive cells, the measuring procedure would produce the digital MTF, not the detector presampling MTF. After recording the system 
response in the moving-slit method, the data are linearized to represent the measured LSF. This linearization is not needed if the digital detector exhibits a fairly linear behavior over the range of exposures selected for the measurement. Several LSF data sets are acquired and averaged, and the measured MTF is then computed (via Eq. 4.43). This MTF is converted to the detector presampling MTF by correcting for the effects of the focal-spot size and the slit width. The correction is done by dividing the measured MTF by the properly-scaled focal-spot and slit MTFs. The focal-spot MTF can be either measured or modeled, e.g., as a double-Gaussian function; ${ }^{46}$ the slit MTF is given by a sinc function, as previously explained. In terms of accuracy, the moving-slit method, similarly to its "analog" counterpart, relies on the proper choice of such experimental factors as the width of the slit, the length of the LSF curve, and the LSF sampling distance.

The moving-edge method, to assess the detector presampling MTF of digital imaging systems with 1D detectors, combines the features of the "analog" edge technique (Section 4.4.1) and the "moving" approach just described for the slit method. Again, the edge is moved across a cell under study while $\mathrm{x}$ rays are on, and the system response from that cell is recorded. This response represents, either directly or after linearization, the measured ESF, which then undergoes the same processing steps as in the "analog" edge technique (i.e., averaging, differentiation, conversion to the measured MTF, and additional MTF correction, if necessary). The measured MTF is also corrected for the effect of the focal spot, to yield the detector presampling MTF. As before, accuracy of the final MTF is influenced by the ESF sampling distance. Overall, the moving-edge method has the same advantages and drawbacks as its "analog" counterpart. 
The presented "moving" approach can also be utilized to measure the detector presampling MTF of imaging systems with 2D discrete detectors. However, the availability of at least several rows of cells in these detectors allows acquisition of necessary data without the need to move a test device (slit or edge). In such acquisition, the test device, instead of being parallel to a column of a 2D detector (i.e., being perpendicular to the direction of the MTF measurement), is slightly angulated with respect to that column. Because of this small angulation, $\mathrm{x}$ rays passing through the center of the slit or along the side of the edge enter the cells in different rows at slightly different lateral positions. By properly combining the readings from several detector rows (as will be discussed later), it is possible to obtain the system response that is sampled at intervals much finer than those provided by the cell-to-cell distance. Hence, for 2D discrete detectors, the required spatial sampling can as well be achieved by angulation, not only by movement, of the test device. Eliminating the movement simplifies and expedites the measuring procedure. For this reason, the methods that use a stationary, angulated (or, in other words, slanted) slit or edge have become the primary choice when measuring the detector presampling MTF of 2D digital systems. It should be noted that the described approach can also be applied to the MTF measurement in the other direction; the roles of columns and rows in that case are interchanged.

The stationary-slanted-slit method, as the name suggests, employs a narrow slit placed at a shallow angle (1.5-3 deg) with respect to cells of a $2 \mathrm{D}$ discrete detector. ${ }^{45,67-70}$ Assume, for specificity, that this angle is formed with a detector column (i.e., the detector presampling MTF is measured in the direction across the columns). During an x-ray exposure, the cells in different rows but within the same column "sample" the system 
response at various distances from the slit center (Fig. 4.3). Each distance is given by the length from the cell center to the slit center. When all such samples in the vicinity of the slit are arranged as a function of this length, the result is the system response sampled at various fractions of the cell-to-cell distance, with the actual sampling intervals small enough to avoid aliasing. According to the format of the stationary-slanted-slit method, the original data are first linearized, if necessary, and then normalized, to correct for variations in x-ray intensity along the slit length. ${ }^{68}$ The normalized data are used to synthesize the finely-sampled measured LSF, where the actual sampling intervals are computed based on the position and angle of the slit. The latter two parameters are accurately determined from the same normalized data. The synthesized measured LSF is next resampled via interpolation to have uniform spatial sampling. ${ }^{69}$ The tails of the resulting LSF are then extrapolated with exponential functions, to extend below the $1 \%$ level. ${ }^{67}$ The final LSF data are transformed to the measured MTF, which, after being corrected for the effects of the focal spot and slit, produces the detector presampling MTF. The required correction steps are the same as mentioned earlier. If possible, the entire MTF measurement is repeated several times, and the results are averaged to improve precision. In general, accuracy of the stationary-slanted-slit method largely depends on the proper angulation of the slit, as this angulation defines the actual LSF sampling distance.

The stationary-slanted-edge method, for measuring the detector presampling MTF of imaging systems with 2D discrete detectors, implements the same angulation approach as the described slit technique but utilizes a sharp edge instead of a slit. ${ }^{45,70-73}$ The edge can be either opaque, essentially absorbing all x rays, - made of a thick tungsten slab - or 

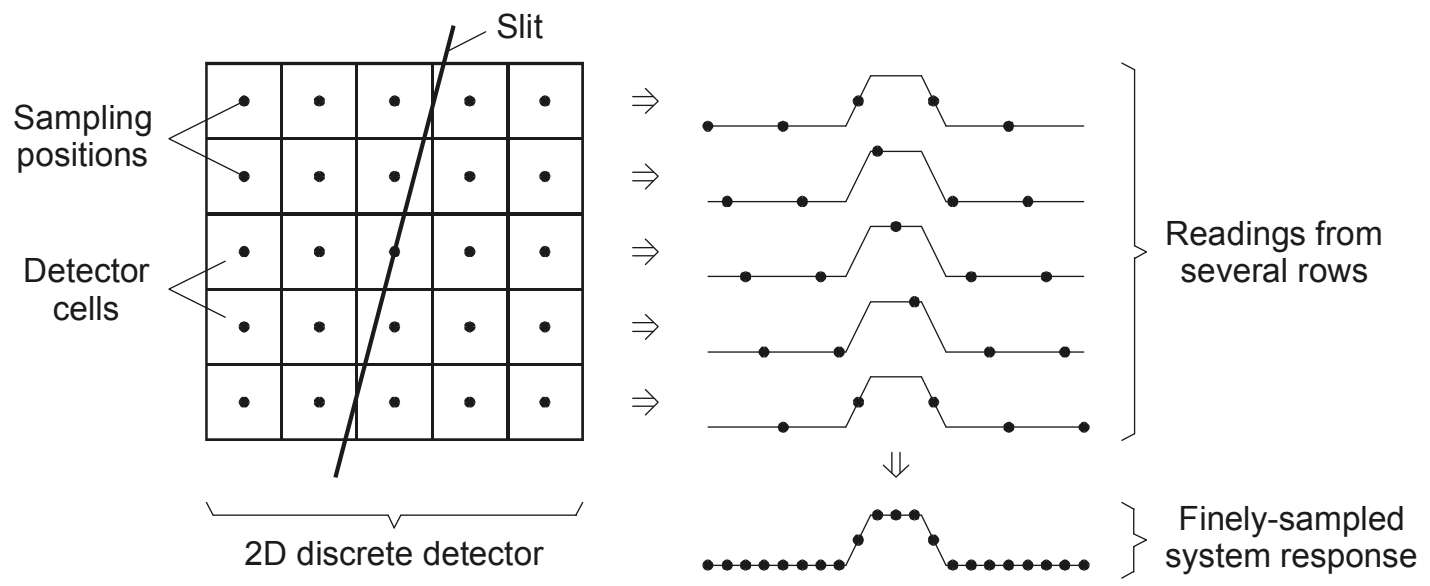

Figure 4.3. Stationary-slanted-slit method to measure the detector presampling MTF.

Source: Adapted with permission. H. Fujita, D. Y. Tsai, T. Itoh, K. Doi, J. Morishita, K. Ueda, and A. Ohtsuka, "A simple method for determining the modulation transfer function in digital radiography," IEEE Trans. Med. Imaging 11, 34-39, 1992. (C) 1992 IEEE. 
translucent, transmitting $10-50 \%$ of incident x rays, - made of a thin platinum or lead foil sandwiched between two acrylic plates. ${ }^{70,71}$ During the measurement, the edge is slightly angulated (1-6 deg) with respect to detector cells, and a necessary image is acquired. The data in the region around the edge, after being adequately pre-processed (linearized, normalized, etc.), are combined to form the sufficiently-sampled measured ESF. Typically, by using the readings from many rows and columns, multiple samples at approximately the same distances from the edge are collected to improve the SNR of the final MTF. ${ }^{71,72}$ These samples are then grouped into small bins whose width corresponds to the actual sampling distance. Such binning ensures uniform spatial sampling. After the binning, the measured ESF is smoothed, numerically differentiated, and converted to the measured MTF. In order to obtain the detector presampling MTF, the latter function is corrected for the effect of the focal spot. Also, the measured MTF has to be corrected for the blurring introduced by almost every data-processing step (i.e., edge-angle determination, data binning, ESF smoothing, and finite-element differentiation). ${ }^{72}$ One of the disadvantages of the stationary-slanted-edge method, in addition to the limitations of the edge technique in general, is difficulty of estimating the edge angle with sufficient accuracy, due to the specifics of the edge image. ${ }^{73}$ As a result, the angle-determination procedure in the stationary-slanted-edge method is more mathematically intense than the similar process in the stationary-slanted-slit technique.

Experimental comparisons of the methods that use stationary slanted test devices to measure the detector presampling MTF of digital imaging systems have produced the results similar to the theoretical findings for the "analog" techniques (these findings were summarized in Section 4.4.1) ${ }^{70,71}$ From the comparisons, the stationary-slanted-slit 
method provides more accurate MTF assessment at high spatial frequencies, due to a large number of x-ray photons contributing to the LSF peak. The stationary-slanted-edge method, on the other hand, gives a better MTF estimate at low frequencies, because of many x-ray photons collected in the regions corresponding to the LSF tails. Overall, when there is no scatter-induced glare in the image, the most accurate measurement of the detector presampling MTF is obtained with the slit. ${ }^{70}$ In the presence of glare, the highest accuracy is achieved with the opaque edge. Also, according to the comparisons, the slit is easier to align, but the slit method is more susceptible to alignment errors. On the contrary, the edge is easier to fabricate, and its physical imperfections have less effect on the final MTF, but the edge method is more sensitive to image noise. The results of the comparisons can as well be extended to the moving-slit and moving-edge techniques, as these procedures employ similar measuring principles. In conclusion, the best way to assess the detector presampling MTF would be to use both the slit and edge methods (with either moving or stationary slanted test devices, depending on the detector type). ${ }^{45}$ If a single method needs to be selected, the choice between the slit and the edge should be made based on the specifics of the measuring task.

\subsubsection{Reconstruction MTF}

Spatial resolution of CT scanners can be measured by the methods that utilize either a PSF, LSF, ESF, or bar phantom. The PSF-phantom method does not have a direct equivalent among the techniques developed for the analog and digital imaging systems. The other three methods are essentially modified versions of the "analog" or "digital" techniques and, therefore, include many steps similar to those explained before. 
The PSF-phantom method measures the reconstruction MTF by determining the response of a CT scanner to a point object represented by a high-density thin wire., ${ }^{2,74-76}$ As long as the diameter of the wire is significantly-smaller than the limiting spatial resolution of the scanner, this response accurately models the "reconstruction" PSF, from which the required MTF can be then computed. ${ }^{2}$ A tungsten or stainless-steel wire, with the diameter of approximately $0.1 \mathrm{~mm}$, is usually employed. The wire is embedded in a test phantom filled with water or other soft-tissue-like material. The phantom is positioned in such a way that the wire lies parallel to the scanner's $z$ axis. The phantom is scanned, and the acquired data are reconstructed with the smallest possible FOV, to ensure adequate spatial sampling. The reconstructed image is corrected for background variations by subtracting a smooth function fitted to the region around the wire. The corrected image corresponds to the reconstruction PSF. This PSF is integrated in one of the image pixel directions (i.e., along the rows, columns, or both) to yield the reconstruction LSF. ${ }^{76}$ From the latter, the reconstruction MTF is computed by means of the Fourier transform (Eq. 4.43). Alternatively, one can first determine the 2D reconstruction MTF, by finding the modulus of the 2D Fourier transform of the reconstruction PSF (Eqs. 4.25 and 4.35). The 1D reconstruction MTF is then simply a profile of the 2D MTF. For many CT scanners, multiple profiles over a 360-deg range can be averaged to give a better estimate of the reconstruction MTF. To further reduce noise, several wires are often scanned simultaneously, and the resulting MTF curves are averaged. Although the PSF-phantom method is fairly straightforward, its accuracy is limited by the pixel size of the digital CT image; the method also uses only a very small fraction of the data produced in the scan. ${ }^{76}$ 
The LSF-phantom method offers a more reliable approach to evaluating the reconstruction MTF of CT scanners. ${ }^{74,76-78}$ This approach is similar to that implemented by the stationary-slanted-slit technique for 2D digital imaging systems (Section 4.4.2). Rather than imaging a slit, the method scans a phantom with a thin metal plate inside. The plate is scanned in the transverse direction, so that the reconstructed image represents the response of a CT scanner to a line object. The utilized LSF phantom can be constructed in several ways. The primary design has been developed by the American Association of Physicists in Medicine (AAPM), in an effort to standardize spatialresolution measurements for CT scanners. According to the AAPM design, the LSF phantom is a disk of solid acrylic that sandwiches a $76-\mu \mathrm{m}$ thick copper foil between the two halves (Fig. 4.4) ${ }^{77}$ A simpler version of the LSF phantom can be built with a thin $(50 \mu \mathrm{m})$ aluminum foil sandwiched between two slabs of acrylic or a similar material. ${ }^{76}$ In either case, the phantom is placed in the scanner gantry, with the foil being orthogonal to the scan plane as well as being slightly angulated (1-8 deg) relative to the horizontal position. The phantom is scanned with the highest $\mathrm{x}$-ray tube current and the narrowest slice thickness. After reconstruction (again, with the smallest possible FOV) and necessary corrections, one obtains an image whose profiles across the foil contain samples of the reconstruction LSF. Due to foil angulation, different profiles include LSF samples at various distances from the foil. Multiple profiles are then combined to provide the finely-sampled, averaged reconstruction LSF. This function is next converted to the reconstruction MTF. To improve precision, the described procedure is repeated several times for the same phantom, and the results are averaged.

The ESF-phantom method to measure the reconstruction MTF is based on the 


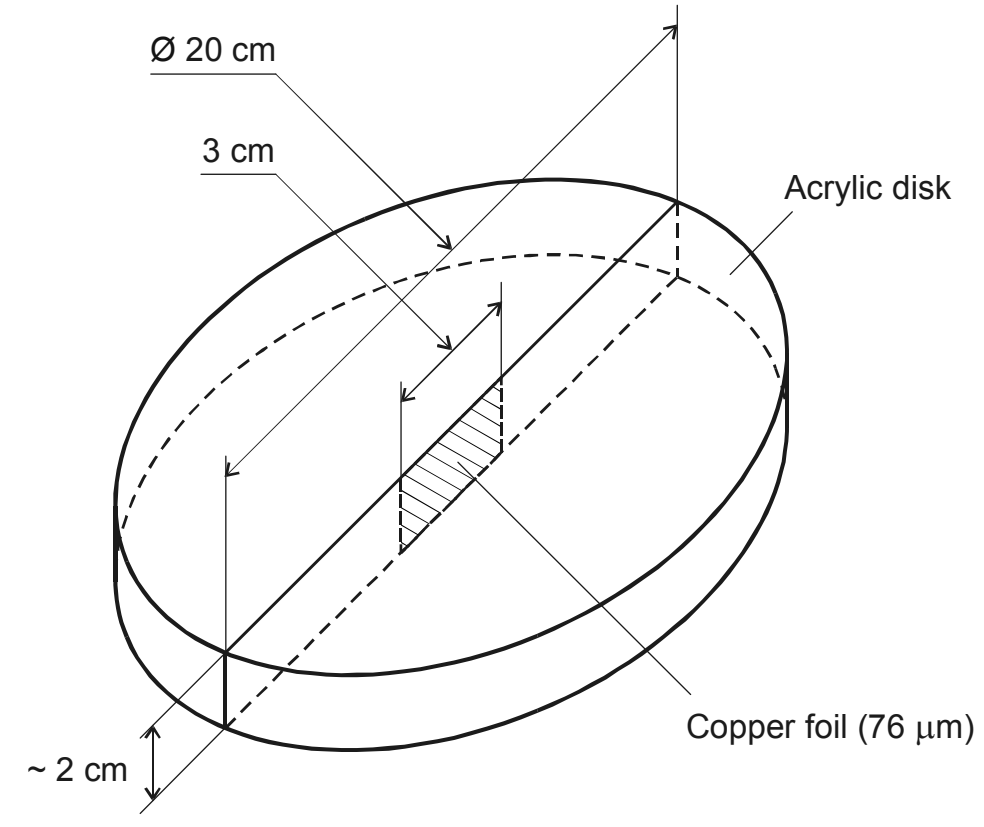

Figure 4.4. AAPM LSF phantom. 
same principle as the previous technique but employs a phantom that simulates an edge instead of a line. ${ }^{74,79}$ The ESF phantom can be implemented by placing two materials of different $\mathrm{x}$-ray attenuation side by side. This arrangement is typically achieved by inserting a block of plastic in a water bath. During the scan, the edge is tilted to form a small angle with the image pixel matrix. The acquisition, reconstruction, and processing steps for the ESF-phantom method are almost the same as for the LSF-phantom technique. The only difference is that the profiles taken from the final image across the edge correspond to the reconstruction ESF, which needs to be differentiated before computing the reconstruction MTF. One advantage of using the ESF phantom in CT is avoidance of streak artifacts frequently seen in LSF-phantom images because of the presence of a high-attenuation metal foil.

The bar-phantom method allows relatively simple and quick evaluation of spatial resolution of CT scanners. Although this method is mainly utilized for qualitative assessment of the scanner resolution, it can also produce a quantitative estimate of the reconstruction MTF. ${ }^{2,80}$ Similarly to its "analog" counterpart (Section 4.4.1), the method scans a cyclic square-wave bar pattern. As in the case of the LSF and ESF phantoms, the bar pattern is slightly angulated with respect to the pixel rows or columns. The image of the bar pattern is reconstructed and then analyzed to determine modulation of the different bars in the phantom. The resulting data represent the values of the CTF at spatial frequencies defined by the size of the bars. The values corresponding to the same-size bars are averaged to reduce noise. The CTF is finally converted to the reconstruction MTF via Coltman's formula (Eq. 4.50). The bar-phantom method, despite its obvious practical advantages, suffers from the same accuracy limitations as the "analog" bar- 
pattern technique.

Although there has been no known comparison of the methods to measure spatial resolution of CT scanners, it is reasonable to expect that the distinctions observed among the measuring techniques for analog and digital imaging systems would exist for the phantom methods as well. Specifically, the LSF-phantom method probably provides a more accurate estimate of the reconstruction MTF at high spatial frequencies, whereas the ESF-phantom method is most likely to give better results at low frequencies. The PSF-phantom method can be considered inferior in terms of noise, and the bar-phantom method, despite its convenience, is expected to be the least accurate. 


\section{CHAPTER 5. METHODS FOR MODELING AND MEASUREMENT OF SPATIAL RESOLUTION IN A VARIABLE RESOLUTION X-RAY COMPUTED TOMOGRAPHY SCANNER*}

This chapter discusses the specifics of studying spatial resolution in a VRX CT scanner, gives a general overview of the current study, presents the design of the experimental scanner, explains the choice of the scanner parameters, and describes the methods used in the study to evaluate two important components of spatial resolution of the experimental VRX CT scanner - the detector presampling MTF and the scanner reconstruction MTF.

\subsection{Specifics and overview of the study}

A VRX CT scanner was, by definition, an imaging system. Its spatial resolution, therefore, could be described within the conceptual framework developed in the previous chapter. However, because of the unique design of this device compared with conventional CT systems, several specific features of the scanner had to be considered when describing its spatial resolution.

First, the VRX CT scanner, in its exact interpretation, only partly satisfied the linearity and shift-invariance conditions required for the spatial-resolution analysis on the basis of transfer theory (Section 4.1). There was no problem with linearity - when employing a solid-state VRX detector, whose response had been found linear in the range

\footnotetext{
* Sections 5.3-5.5 of this chapter adapted with permission. R. Melnyk and F. A. DiBianca, "Modeling and measurement of the detector presampling MTF of a variable resolution x-ray CT scanner," Med. Phys. 34, 1062-1075, 2007.
} 
of exposures typically used for spatial-resolution measurements, ${ }^{33,40}$ this requirement was easily met, and no additional linearization of the scanner was needed. But the situation was different with the shift-invariance condition. The VRX CT scanner was not shiftinvariant over the entire object plane. The main reason for this included angulation of the VRX detector. Because of the angulation, combined with the fan-beam geometry of the scanner, the cells at different positions from the detector vertex produced different responses when imaging the same object. Another reason was the discrete nature of the VRX detector. This property led to the same shift-variance that is often observed in digital imaging systems (Section 4.3). Despite the mentioned difficulties, the shiftinvariance condition for the VRX CT scanner was approximated by considering each cell separately and then presenting the overall response of the scanner as a function of the cell position. Thus, with the latter approximation, both transfer-theory requirements were satisfied, and the resolution properties of the scanner were analyzed in terms of the previously established measures of spatial resolution.

Next, the VRX CT scanner, according to its underlying principle, provided a resolution increase in the scan $(x-y)$ direction only. The type of the scanner presented in the current study also employed a 1D detector, which was sufficient for proper implementation of the VRX detection technique (Section 3.1). Consequently, at the detector level, spatial resolution was described using 1D measures (the LSF and 1D MTF). The same 1D measures were also chosen to characterize spatial resolution near the center of the final, reconstructed image, as this resolution was believed to be isotropic. Another specific feature of the VRX CT scanner was an asymmetrical response from each detector cell. This resulted, again, from the angulation of the VRX detector. 
Due to the angulation, there was a difference between the left and right tails of the corresponding LSF. Clearly, such LSF asymmetry would affect the phase information in the spatial-frequency domain. Although the phase content of the scanner spatial resolution was not separately considered, the effect of the LSF asymmetry on the detector MTF was carefully examined.

Finally, the VRX CT scanner exhibited larger noise variations and might be subjected to slightly more noise at small FOVs than conventional CT systems. The stochastic nature of this noise, however, was generally ignored, and the resolution properties of the scanner were described by the parameters representing only the expectation values of the selected spatial-resolution measures. The sole statistical effects accounted for in the study were simple variations among different samples of the same parameters.

In an overview, the goal of the current study was a comprehensive evaluation of spatial resolution in the VRX CT scanner. Two components of this resolution were considered - the pre-reconstruction (before image reconstruction) spatial resolution and the post-reconstruction (after image reconstruction) spatial resolution. The postreconstruction spatial resolution was chosen for the evaluation because of the importance of this parameter from the clinical point of view. Indeed, this type of spatial resolution described the quality of an image at the final stage of the scanner imaging chain; that image would be used by a radiologist to make a clinical decision. The pre-reconstruction spatial resolution, on the other hand, was selected for the evaluation because of its significance from the engineering point of view. Specifically, the pre-reconstruction spatial resolution characterized the performance of the VRX detector itself, without 
influence of the reconstruction algorithm; this parameter, therefore, provided better understanding of the resolution improvement resulting from the detector angulation.

Both components of spatial resolution of the VRX CT scanner were evaluated in terms of the MTF. Based on the MTF hierarchy developed in the previous chapter (Fig. 4.2), the post-reconstruction spatial resolution was represented by the scanner reconstruction MTF. The choice of a measure for the pre-reconstruction spatial resolution was less obvious. Among the three available options - the detector presampling MTF, the system presampling MTF, and the scanner digital MTF - the first measure was selected, due to its advantages when describing inherent spatial resolution of discrete detectors (Section 4.3). Hence, the pre-reconstruction spatial resolution of the VRX CT scanner was given by the detector presampling MTF. On the whole, the detector presampling MTF and the scanner reconstruction MTF were the two component-specific measures of spatial resolution evaluated in the study.

An initial intention was to determine each MTF via both modeling and measurement, and then compare the results to asses the adequacy of the modeling approach. The two distinct ways to obtain the same measure would also provide means for result verification. This idea was successfully realized for the detector presampling MTF, which was modeled by the Monte Carlo technique and measured by the movingslit method. In the case of the scanner reconstruction MTF, however, the computation of this function from the modeled detector presampling MTF, as originally planned, was found to be difficult. Therefore, the scanner reconstruction MTF was only measured, using the LSF-phantom method. 


\subsection{Experimental VRX CT scanner}

The primary focus of the current study was the experimental VRX CT scanner, whose resolution properties were thoroughly evaluated. As mentioned in Sections 3.4 and 3.5, the experimental scanner (Fig. 5.1) employed a 576-channel solid-state dual-arm VRX detector that operated in the symmetrical (normal) mode. The scanner also included an X-ray tube with its own collimator, an additional slice-thickness collimator, a motorized rotary table, low-noise detector electronics, and several data-acquisition and system-control computers. Both the VRX detector and the x-ray tube were kept stationary during a scan, while an object being imaged was placed on the rotary table and rotated around its vertical axis.

The VRX detector was built in house with the help of Biomedical Instrumentation Division at the University of Tennessee Health Science Center. The detector consisted of two aluminum frames that could rotate around a common pivotal point (vertex). The frames enclosed custom-fabricated linear scintillator-photodiode arrays (UDT Sensors Inc., Hawthorne, CA), referred to as detector modules. The front surfaces of all the modules on each frame were aligned with a plane passing through the detector vertex. The modules were also tightly packed to avoid any gaps between them within the frames and, if possible, at the vertex between the frames. Each aluminum frame with the installed scintillator-photodiode arrays represented one arm of the VRX detector. Exact details of the detector design will be discussed later in this chapter.

The VRX detector was placed on a steel support table that had a preset position for the detector vertex. By sliding the free ends of the detector arms on the table, one could rotate the arms around the vertex and, thus, angulate the detector. For convenience, 


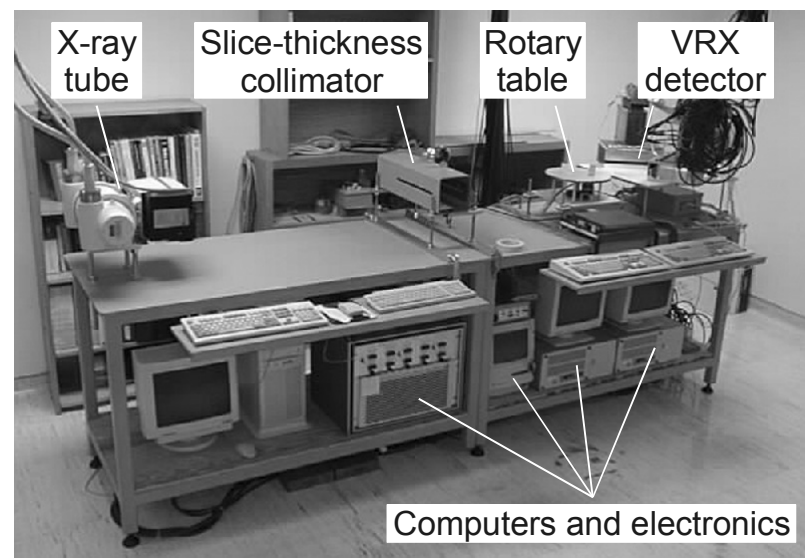

Figure 5.1. Experimental VRX CT scanner.

Source: Reprinted with permission. R. Melnyk and F. A. DiBianca, "Modeling and measurement of the detector presampling MTF of a variable resolution x-ray CT scanner," Med. Phys. 34, 1062-1075, 2007. 
the table had a grid of opening half-angles etched on its top surface. The support table was positioned at one end of a 3-m long steel bench; if needed, the location of the table on the bench could be easily changed. The other end of the bench included a Varian G1582BI rotating anode x-ray tube in a B180H housing (Varian Medical Systems, Salt Lake City, UT). To increase x-ray output, the tube was used with a Varian HE100 heat exchanger and a Lytron MCS20H03M03 modular cooling system (Lytron Inc., Woburn, MA). The position of the tube on the bench was fixed in the longitudinal direction but could be adjusted in the lateral direction. The tube employed a Eureka Linear I x-ray collimator (Eureka X-ray Tube Co., Chicago, IL) attached to the tube front window. This collimator limited the x-ray beam mainly in the horizontal direction. The beam size in the vertical direction was accurately set by the slice-thickness collimator, placed between the x-ray tube and an object being imaged. The slice-thickness collimator was also built in house; it provided a micrometer-controlled opening in the range of 0-7 $\mathrm{mm}$, with accuracy of $0.01 \mathrm{~mm}$. The final component located on the top of the steel bench was a Techno-Isel Model 5 stepper-motor rotary table (Techno-Isel, New Hyde Park, NY). This table was installed between the slice-thickness collimator and the VRX detector. The rotary table had four adjustable brackets on its top plate to support an object. Overall, the detector could be positioned as far as $2.6 \mathrm{~m}$ from the x-ray tube (when measuring from the detector vertex to the tube focal spot). The slice-thickness collimator and the rotary table could be placed anywhere between the detector and the tube. The positions of the four described components could also be adjusted in the vertical as well as lateral directions, to allow proper mechanical alignment of the experimental VRX CT scanner. The detector electronics was originally custom-designed for a different imaging 
system but was modified to meet requirements of the experimental VRX CT scanner. The electronics included 12 amplifier boards (each with 48 current integrators), multiplexing circuitry, 12 analog-to-digital conversion boards (Analogic Corporation, Peabody, MA), a control module, and a power supply. The amplifier boards and the multiplexing circuitry were mounted on the arms of the VRX detector. The analog-to-digital conversion boards were installed in two specially assembled 80486-based dataacquisition computers. The control module was connected to a PowerPC-based machine. A separate Pentium-based computer controlled the operation of the rotary table, and a high-performance Pentium-based workstation (located outside the scanner's steel bench) was used to store and reconstruct acquired image data.

\subsection{VRX CT scanner parameters}

In order to model and measure spatial resolution of the experimental VRX CT scanner, a number of parameters were selected and computed. These included detector parameters, scanner geometrical parameters, and optimum x-ray beam parameters.

\subsubsection{Detector parameters}

The dual-arm VRX detector of the experimental VRX CT scanner utilized 24 (12 per arm) linear scintillator-photodiode arrays, or detector modules. Each module consisted of 24 cadmium tungstate $\left(\mathrm{CdWO}_{4}\right)$ scintillator crystals, or cells (Fig. 5.2). The cells in every module were separated by inner lead $(\mathrm{Pb})$ separators. Two outer lead separators, of the width different from that of inner separators, were placed on the left and right ends of a detector module. Also, there was aluminum oxide $\left(\mathrm{Al}_{2} \mathrm{O}_{3}\right)$ reflective- 


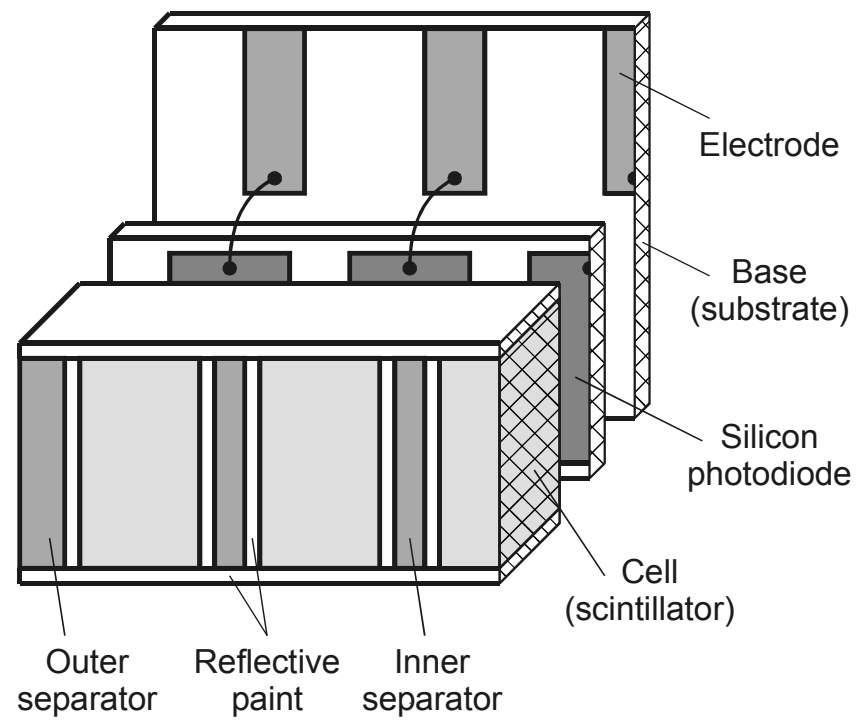

Figure 5.2. Section of a VRX detector module.

Source: Reprinted with permission. R. Melnyk and F. A. DiBianca, "Modeling and measurement of the detector presampling MTF of a variable resolution x-ray CT scanner," Med. Phys. 34, 1062-1075, 2007. 
paint coating between the cells and the separators in a module. The same coating was present on the top and bottom of every module. The main parameters of the VRX detector are given in Table 5.1.

\subsubsection{Scanner geometrical parameters}

Geometrical parameters of the experimental VRX CT scanner were selected and computed based on its schematic diagram (Fig. 5.3), which was developed from the diagram of the typical VRX CT scanner (Fig. 3.4). There were three groups of geometrical parameters: (1) constant parameters, (2) an independent parameter, and (3) dependent parameters. The constant parameters comprised the source-vertex distance, the source-object distance, and the active arm length. These three parameters were the same throughout the study. The independent parameter was the FOV; it was varied in the range from 1 to $32 \mathrm{~cm}$, and the scanner spatial resolution was modeled and measured as a function of this FOV. The dependent parameters included the opening half-angle, the incident angle, and the source-cell distance. These parameters were computed as functions of the FOV. The two latter parameters were also functions of the cell under study. A list of the primary scanner geometrical parameters is given in Table 5.2.

The active arm length was computed assuming that only 256 out of 288 cells on each arm of the VRX detector were used to image an object. These were the active cells. The remaining 32 cells at the end of each arm were the reference cells; they were used to correct for variations in the x-ray tube output. The number of active/reference cells was selected based on an acceptable maximum value $(1.18 \%)$ of the error in corrected airscan images. This error was defined as the standard deviation divided by the mean of the 
Table 5.1. Parameters of the VRX detector.

\begin{tabular}{lc}
\hline \multicolumn{1}{c}{ Parameter } & Value \\
\hline Number of cells per module & 24 \\
Number of modules & $12+12$ \\
Total number of cells & 576 \\
Cell material & $\mathrm{CdWO}_{4}$ \\
Separator material & $\mathrm{Pb}$ \\
Reflective-paint material & $\mathrm{Al}_{2} \mathrm{O}_{3}$ \\
Cell width & $0.79 \mathrm{~mm}$ \\
Inner-separator width & $0.10 \mathrm{~mm}$ \\
Outer-separator width & $0.18 \mathrm{~mm}$ \\
Reflective-paint width & $0.05 \mathrm{~mm}$ \\
Cell height & $20.14 \mathrm{~mm}$ \\
Cell thickness & $3.00 \mathrm{~mm}$ \\
\hline
\end{tabular}

Source: Adapted with permission. R. Melnyk and F. A. DiBianca, "Modeling and measurement of the detector presampling MTF of a variable resolution x-ray CT scanner," Med. Phys. 34, 1062-1075, 2007. 


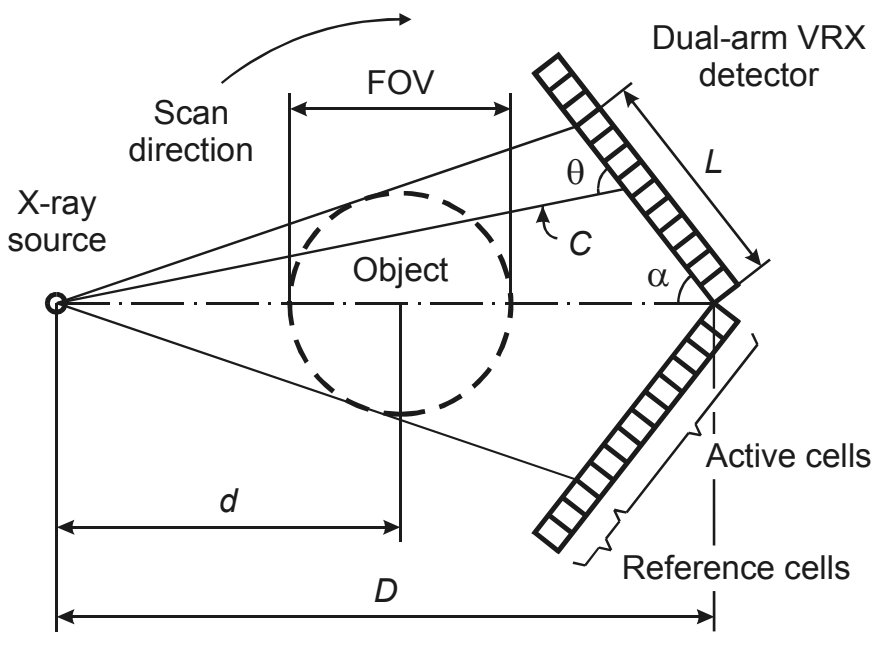

Figure 5.3. Schematic diagram of the experimental VRX CT scanner: $D$ - source-vertex distance, $d$ - source-object distance, $C$ - source-cell distance, $L$ - active arm length, $\alpha-$ opening half-angle, and $\theta$-incident angle. FOV is the field of view.

Source: Adapted with permission. R. Melnyk and F. A. DiBianca, "Modeling and measurement of the detector presampling MTF of a variable resolution x-ray CT scanner," Med. Phys. 34, 1062-1075, 2007. 
Table 5.2. Geometrical parameters of the experimental VRX CT scanner.

\begin{tabular}{lc}
\hline \multicolumn{1}{c}{ Parameter } & Value \\
\hline Number of active cells per arm & 256 \\
Number of reference cells per arm & 32 \\
Active arm length & $25.617 \mathrm{~cm}$ \\
Source-vertex distance & $150 \mathrm{~cm}$ \\
Source-object distance & $106 \mathrm{~cm}$ \\
Maximum FOV & $36.21 \mathrm{~cm}$ \\
FOV & $1-32 \mathrm{~cm}$ \\
\hline
\end{tabular}

Source: Adapted with permission. R. Melnyk and F. A. DiBianca, "Modeling and measurement of the detector presampling MTF of a variable resolution x-ray CT scanner," Med. Phys. 34, 1062-1075, 2007. 
corrected (for the channel gain and offset, as well as for the tube output variations) signal from one active cell; the maximum value was obtained by computing and comparing the errors for all active cells.

\subsubsection{X-ray beam parameters}

The X-ray beam parameters of the experimental VRX CT scanner comprised the tube voltage, tube current, exposure time, filtration, and beam thickness. These parameters were computed from a simple theoretical optimization study. The study consisted in minimizing patient dose while maintaining a fixed contrast SNR and limiting the tube heat.

The contrast SNR for one projection, not for a reconstructed image, was considered. The contrast SNR was computed as

$$
\mathrm{SNR}_{C}=\frac{C}{\sigma_{C}},
$$

where $C$ is the contrast, and $\sigma_{C}$ is the standard deviation of the contrast. The contrast was defined by

$$
C=2 \frac{\left|E_{\mathrm{T}}-E_{\mathrm{B}}\right|}{E_{\mathrm{T}}+E_{\mathrm{B}}},
$$

where $E_{\mathrm{T}}$ and $E_{\mathrm{B}}$ are the x-ray energies absorbed in a cell under study after x rays passed through the target and background regions of a simple water phantom, respectively. Assuming these energies were independent, the detector was ideal, the system was quantum-limited, and the signal variations were due to photon-counting (Poisson) statistics only, the following expression was derived for the contrast SNR: 


$$
\mathrm{SNR}_{C}=\frac{\left|E_{\mathrm{T}}^{2}-E_{\mathrm{B}}^{2}\right|}{2 \sqrt{E_{\mathrm{T}}^{2} \sigma_{\mathrm{B}}^{2}+E_{\mathrm{B}}^{2} \sigma_{\mathrm{T}}^{2}}},
$$

where $\sigma_{\mathrm{B}}$ and $\sigma_{\mathrm{T}}$ are the standard deviations corresponding to $E_{\mathrm{B}}$ and $E_{\mathrm{T}}$. Each of these standard deviations was computed according to

$$
\sigma=\sum_{i}^{\text {all energy intervals }} S_{i} \xi_{i}^{2} \Delta \xi
$$

where $S_{i}$ is the $i$-th interval value of the respective absorbed spectrum for the cell under study, $\xi_{i}$ is the mid-interval energy, and $\Delta \xi$ is the energy interval. A threshold contrast SNR of five was assumed for the optimization study.

The patient dose was represented by the "skin" dose, which was the dose for the outer $2 \mathrm{~mm}$ of the same water phantom along the detector centerline (i.e., the line connecting the tube focal spot and the detector vertex). This dose was computed assuming a narrow-beam geometry and scatter in the phantom. ${ }^{7,81}$ In units of cGy, this dose was given by

$$
D=\frac{1.602 \times 10^{-11}}{\ell \rho} \int_{0}^{\xi_{\max }} n \xi\left(1-e^{-\mu \ell}\right)\left(\frac{\mu^{*}}{\mu}+0.1\right) d \xi,
$$

where $\ell$ is the "skin" thickness in $\mathrm{cm}, \rho$ is the "skin" density in $\mathrm{g} / \mathrm{cm}^{3}, \xi_{\max }$ is the maximum energy in $\mathrm{keV}$ of $\mathrm{x}$ rays passing through the phantom, $n$ is the input photon fluence in $1 / \mathrm{cm}^{2}, \xi$ is the mid-interval energy in $\mathrm{keV}, \mu$ and $\mu^{*}$ are the corresponding linear attenuation and energy absorption coefficients, respectively, in $1 / \mathrm{cm}, 0.1$ is the factor describing scatter (according to an assumption ${ }^{81}$ for energies above $50 \mathrm{keV}$ ), and $d \xi$ is the energy interval in $\mathrm{keV}$. The linear attenuation and energy absorption coefficients to compute both the dose in the phantom and the absorbed spectrum in a 
detector cell were taken from the NIST XCOM database. ${ }^{82}$

The beam thickness was set by the slice-thickness collimator placed at $70 \mathrm{~cm}$ from the x-ray tube focal spot. The collimator opening was proportional to the FOV, with the scale factor of 0.02 . The optimization was done for cell \#128, which was the middle active cell on a detector arm.

A cylindrical water phantom was assumed for the study (Fig. 5.4). The diameter of the phantom was equal to the FOV. As mentioned before, the contrast was formed by $\mathrm{x}$ rays passing through two different regions of the phantom. In the background region, $\mathrm{x}$ rays were attenuated only by water $\left(\mathrm{H}_{2} \mathrm{O}\right)$ with the density of $1.0 \mathrm{~g} / \mathrm{cm}^{3}$. In the target region, $\mathrm{x}$ rays passed through the same water background plus a thin target, which had different attenuation properties than the water background. The diameter of the target was FOV/100. Two types of the target were considered. The first type consisted of water too, but with the density of $0.9 \mathrm{~g} / \mathrm{cm}^{3}$. This target provided the contrast based on the density difference, and the corresponding phantom represented a simplified "tissue" phantom. In the second type of the target, $1 \%$ of calcium $(\mathrm{Ca})$ by mass was added to water with the density of $1.0 \mathrm{~g} / \mathrm{cm}^{3}$. The latter target provided the contrast based on the atomic number difference. The phantom with this target represented a simplified "bone" phantom.

The use of water (simulating normal biological soft tissue) and calcium (representing the most attenuating biological substance appreciably present in mammals) in the optimization study was believed to set low and high bounds on the optimum tube voltage. Also, in the author's opinion, simple mechanical phantoms (e.g., the simplified "tissue" and "bone" phantoms) containing materials that represented the attenuation limits of biological subjects were preferable, for general system analysis, to complex, 


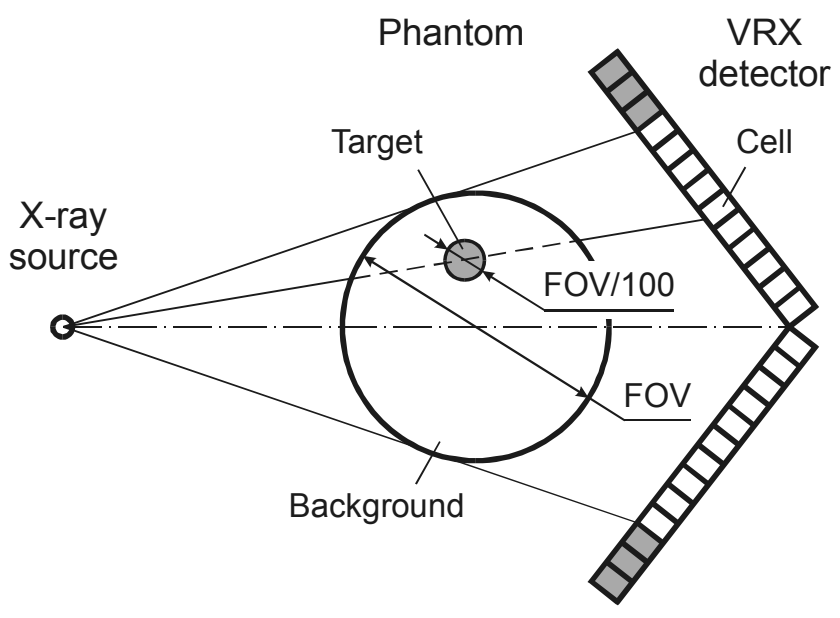

Figure 5.4. Theoretical phantom for computing optimum $x$-ray beam parameters. FOV is the field of view. 
task-based anthropomorphic phantoms.

To simplify the optimization procedure, no external filter was considered. Only constant internal filtration consisting of $1 \mathrm{~mm}$ of aluminum (Al) in the $\mathrm{x}$-ray tube and $2 \mathrm{~mm}$ of aluminum equivalent in the tube collimator was used. Under the condition of having the contrast SNR of five, the skin dose was computed as a function of the tube voltage at each FOV. The tube voltage that provided the minimum skin dose was selected as the optimum tube voltage at a particular FOV. Because there was no external filter, the resulting tube heat for the typical exposure time (4 s) was below the allowable limits for the Varian G1582BI x-ray tube $(0.6 \mathrm{~mm}$ nominal focal spot; active cooling as described in Section 5.2) used in the experimental VRX CT scanner. ${ }^{83}$ Therefore, no additional limiting of the tube heat (hence, the tube current) was required.

The computed optimum tube voltage was found independent of the target density, the fraction of calcium in the target, and the target thickness (diameter). The target density was varied in the $0.1-0.9 \mathrm{~g} / \mathrm{cm}^{3}$ range for the "tissue" phantom, whereas the mass fraction of calcium was changed in the 1-5\% range for the "bone" phantom. Also, the FOV-to-target diameter ratio was varied from 5 to 100 for both "tissue" and "bone" phantoms. In all cases, the changes only shifted the dose curve up or down on the $y$ axis without affecting its shape, thus without changing the corresponding optimum tube voltage. The independence of the optimum tube voltage on the target diameter provided strong evidence that spatial-frequency-dependent effects were absent in the cases examined.

Plots of the optimum tube voltage as a function of the scanner FOV for the "tissue" and "bone" phantoms are shown in Fig. 5.5. Because the operating-voltage range 


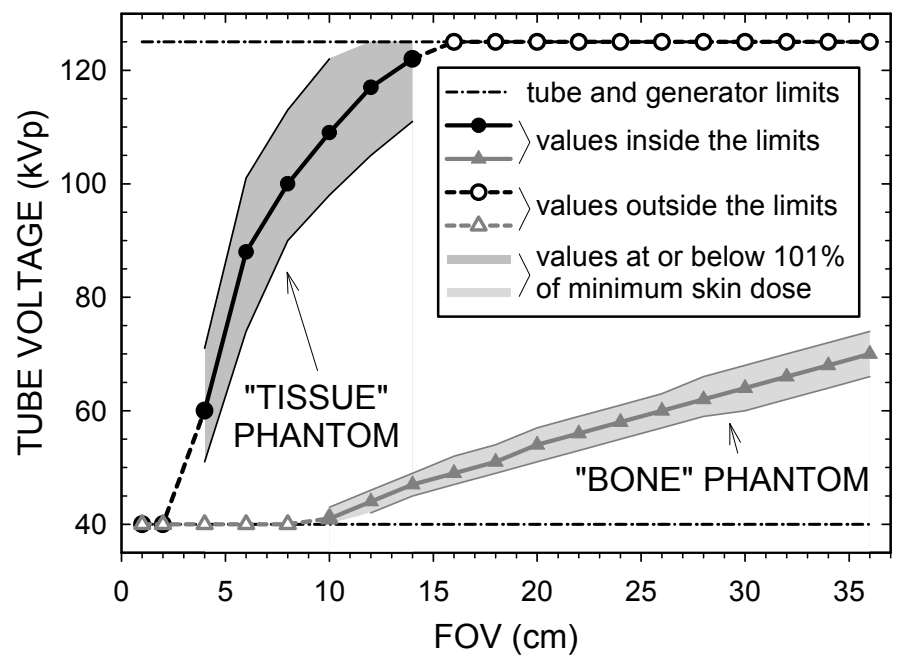

Figure 5.5. Optimum $x$-ray tube voltage for the experimental VRX CT scanner.

Source: Reprinted with permission. R. Melnyk and F. A. DiBianca, "Modeling and measurement of the detector presampling MTF of a variable resolution x-ray CT scanner," Med. Phys. 34, 1062-1075, 2007. 
for the $\mathrm{x}$-ray tube and generator was $40-125 \mathrm{kVp}$, when the optimum tube voltage fell outside this range, the corresponding limiting voltage was used instead of the optimum voltage. The values of the optimum tube voltage, along with the other main parameters utilized to model and measure spatial resolution of the experimental VRX CT scanner, are given in Table 5.3.

An attempt was also made to optimize the tube voltage using an external copper $\mathrm{C} \mathrm{Cu}$ filter placed between the X-ray tube and the phantom. This approach was more complicated than the optimization procedure discussed previously because it considered the heating-cooling properties of the employed x-ray tube. As a result of the tube heat limitations, the external-filter approach produced not only the optimum tube voltage but also the corresponding values of the optimum tube current and the external-filter thickness at each FOV. Although the presence of the external filter implied lower patient dose, this approach turned out to be extremely complex and time-consuming. Besides, the actual skin dose in the external-filter approach was found to be only $15 \%$ or less lower than the dose in the optimization procedure with no external filter. Therefore, the approach involving the external copper filter was deemed impractical and was not further pursued.

\subsection{Modeling of the detector presampling MTF}

The detector presampling MTF, which described the pre-reconstruction spatial resolution of the experimental VRX CT scanner, was modeled by the Monte Carlo simulation. Because the VRX detector was a 1D discrete detector, the simulation was based on the moving-slit method used to measure the detector presampling MTF of 
Table 5.3. Modeling and measurement parameters for the experimental VRX CT scanner.

\begin{tabular}{|c|c|c|c|c|c|c|}
\hline \multirow{2}{*}{$\begin{array}{l}\text { FOV } \\
(\mathrm{cm})\end{array}$} & \multirow{2}{*}{$\begin{array}{c}\text { Opening } \\
\text { half-angle } \\
\text { (deg) }\end{array}$} & \multicolumn{3}{|c|}{ Incident angle (deg) } & \multicolumn{2}{|c|}{ Optimum tube voltage $(\mathrm{kVp})$} \\
\hline & & $\begin{array}{c}\text { Cell } \\
\# 1\end{array}$ & $\begin{array}{c}\text { Cell } \\
\# 128\end{array}$ & $\begin{array}{c}\text { Cell } \\
\# 256\end{array}$ & $\begin{array}{l}\text { "Tissue" } \\
\text { phantom }\end{array}$ & $\begin{array}{l}\text { "Bone" } \\
\text { phantom }\end{array}$ \\
\hline 1 & 1.31 & 1.31 & 1.43 & 1.58 & {$[40]$} & {$[40]$} \\
\hline 2 & 2.63 & 2.63 & 2.87 & 3.17 & [40] & [40] \\
\hline 4 & 5.26 & 5.26 & 5.75 & 6.34 & 60 & [40] \\
\hline 8 & 10.60 & 10.61 & 11.58 & 12.76 & 100 & [40] \\
\hline 16 & 21.90 & 21.91 & 23.87 & 26.22 & {$[125]$} & 49 \\
\hline 32 & 53.43 & 53.45 & 57.55 & 62.09 & {$[125]$} & 66 \\
\hline
\end{tabular}

Note: Values in square brackets are the tube and generator limits when actual optimum tube voltages fall outside these limits.

Source: Adapted with permission. R. Melnyk and F. A. DiBianca, "Modeling and measurement of the detector presampling MTF of a variable resolution x-ray CT scanner," Med. Phys. 34, 1062-1075, 2007. 
digital imaging systems with 1D detectors (Section 4.4.2). Accordingly, the MTF modeling was done by “moving," with a very small step, a perfect (zero-thickness) pencil x-ray beam along the VRX detector and simulating the energy deposited in a cell under study as a function of the beam position. This energy represented the detector presampling LSF, from which the corresponding MTF was then computed. Because of the ability to use a perfect pencil beam in the Monte Carlo program, there was no need to simulate a slit.

\subsubsection{VRX detector model}

The VRX detector model was the same as the one utilized in the Monte Carlo study of x-ray cross-talk in the VRX detector. ${ }^{36}$ The model followed the design of the actual VRX detector, with the main parameters given in Table 5.1.

The basic component of the model was a cadmium tungstate $\left(\mathrm{CdWO}_{4}\right)$ cell represented by a rectangular parallelepiped of width CellWidth, height CellHeight, and thickness CellThick (Fig. 5.6-a). Twenty-four cells composed one detector module. The cells were separated by lead $(\mathrm{Pb})$ separators. Inner separators of width SepIWidth were placed between the cells in the module. Two outer separators of width SepOWidth were placed on the left and right ends of the module. Between the cells and the separators were gaps corresponding to aluminum oxide $\left(\mathrm{Al}_{2} \mathrm{O}_{3}\right)$ reflective-paint coating that was present in the actual detector but not considered in the model. The width of these gaps was PaintWidth. The module also included an aluminum oxide base of thickness BaseThick behind the cells (Fig. 5.6-b).

In the model, 12 detector modules placed side-by-side formed one arm of the 


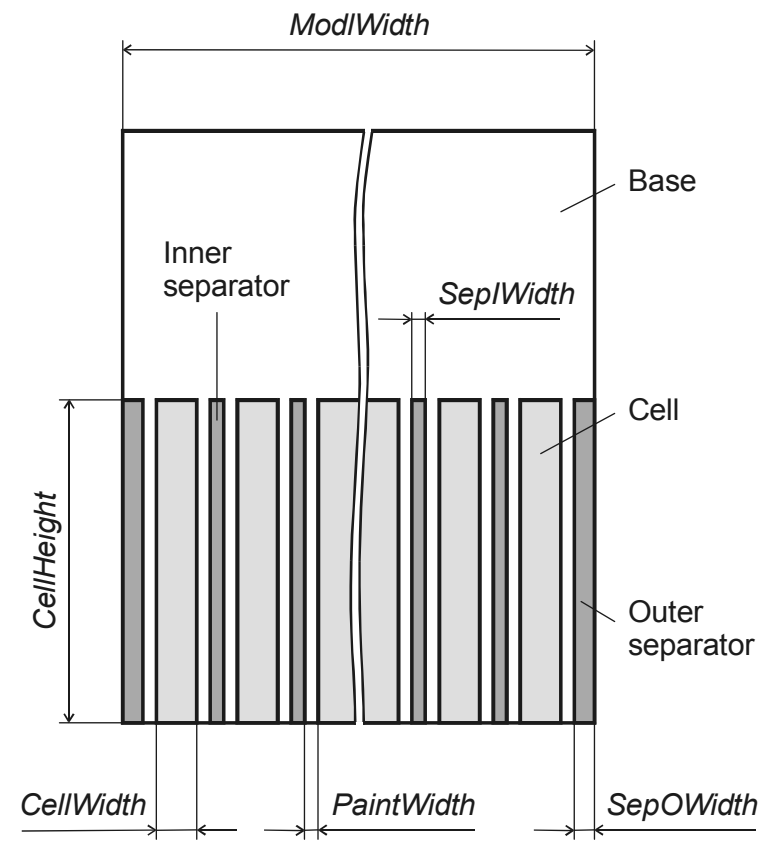

(a)

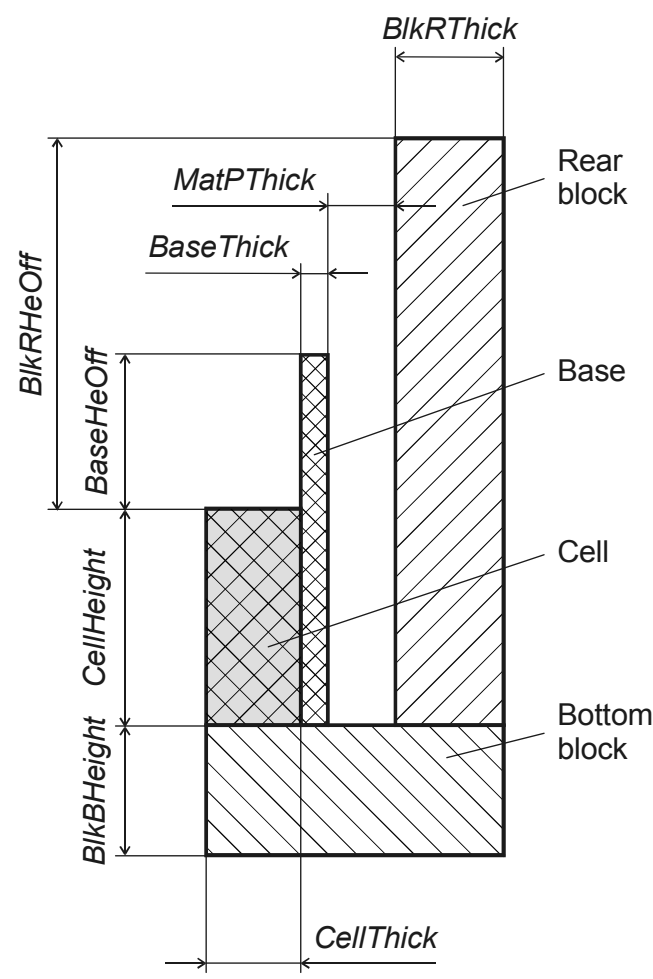

(b)

Figure 5.6. VRX detector model: (a) detector module, (b) cross-section of a detector arm.

Source: Adapted with permission. R. Melnyk and F. A. DiBianca, "Monte Carlo study of x-ray cross-talk in a variable resolution x-ray detector," Proc. SPIE 5030, 694-701, 2003. 
VRX detector. Only one module with discrete cells was used; all the other modules on that arm and all the modules on the opposite arm were replaced by continuous blocks from a uniform material. This material was a mixture of the cell and separator materials combined in such a way that the continuous blocks had the same x-ray attenuation properties as the replaced discrete modules. For that purpose, the weight fraction of each chemical element in the cell material was multiplied by the cell-width fraction (the fraction of the module width occupied by the cells). Similarly, the weight fraction of each element in the separator material was multiplied by the separator-width fraction (the fraction of the module width occupied by the separators). The density of the uniform material was the sum of the cell- and separator-material densities, each multiplied by the corresponding width fraction.

The metal frames that supported the detector modules in the actual VRX detector were represented in the model by aluminum (Al) blocks, two for each arm (Fig. 5.6-b). The bottom block of height BlkBHeight was positioned right under the modules, in accordance with the design of the actual detector. The rear block of thickness BlkRThick was placed at a small distance behind the modules. The gap between the rear block and the module base corresponded to a porous material found in the actual VRX detector. The thickness of this gap was MatPThick. Numerical values of the parameters of the VRX detector model are given in Table 5.4.

\subsubsection{Monte Carlo simulation}

The 3D, ACCEPTP ITS 3.0 Monte Carlo code was used for the simulation. ${ }^{84}$ This code provided a state-of-the-art Monte Carlo solution of linear time-independent coupled 
Table 5.4. Parameters of the VRX detector model.

\begin{tabular}{llr}
\hline \multicolumn{1}{c}{ Parameter } & Notation & Value (mm) \\
\hline Cell width & CellWidth & 0.79 \\
Cell height & CellHeight & 20.14 \\
Cell thickness & CellThick & 3.00 \\
Inner-separator width & SepIWidth & 0.10 \\
Outer-separator width & SepOWidth & 0.18 \\
Reflective-paint width & PaintWidth & 0.05 \\
Module width & ModlWidth & 24.02 \\
Base thickness & BaseThick & 1.02 \\
Base height offset & BaseHeOff & 15.70 \\
Bottom-block height & BlkBHeight & 19.00 \\
Rear-block thickness & BlkRThick & 11.00 \\
Rear-block height offset & BlkRHeOff & 56.00 \\
Porous-material thickness & MatPThick & 3.00 \\
\hline
\end{tabular}

Source: Adapted with permission. R. Melnyk and F. A. DiBianca, "Monte Carlo study of $\mathrm{x}$-ray cross-talk in a variable resolution x-ray detector," Proc. SPIE 5030, 694-701, 2003. 
electron/photon radiation transport problems, with or without the presence of macroscopic electric and magnetic fields. Physical rigor was maximized by employing some of the best available cross sections and sampling distributions, as well as the most complete existing physical model for describing the production and transport of the electron/photon cascade from $1.0 \mathrm{GeV}$ down to $1.0 \mathrm{keV}$.

The Monte Carlo code was run in the photon-only transport mode. This mode substantially increased the simulation speed while producing results with no significant differences from the results obtained in the coupled electron/photon transport mode, as was verified in several test runs. A polychromatic point x-ray source was used to create a perfect (zero-thickness) pencil beam. The source spectra were generated by the method of Birch and Marshall. ${ }^{85}$ This method has been shown to produce spectra that were not significantly-different from experimental spectra ${ }^{86}$ For the spectrum generation, the target angle of the anode of $10 \mathrm{deg}$ and the internal aluminum (Al) filtration of $3 \mathrm{~mm}$ were chosen, according to the parameters of the x-ray tube and tube collimator utilized in the experimental VRX CT scanner. ${ }^{83}$ To verify that the generated spectra were consistent with the actual spectra used for the MTF measurement, the corresponding half-value layers (HVLs) were determined and compared. For the tube voltage of $80 \mathrm{kVp}$ and no external filtration, both the theoretical and experimental HVLs were found to be equal to $6.0 \mathrm{~mm}$ of aluminum within an uncertainty of $\pm 0.4 \mathrm{~mm}$.

To simulate the detector presampling MTF, only one arm of the VRX detector was considered. The simulation was done at six opening half-angles, corresponding to the six selected FOVs (Table 5.3). At each opening half-angle, the simulation was repeated for the following three cells: \#1 (vertex cell), \#128 (middle active cell), and \#256 (last 
active cell). At 1 and 2-cm FOVs, the simulation was also repeated for cell \#283, in order to compare the modeling results with results of the measurement, which could only be made for cell \#283 because of this cell's lower system magnification (which helped avoid the effect of the focal-spot size). For each of the mentioned cells, the simulation was repeated twice, with the values of the optimum tube voltage for the "tissue" and "bone" phantoms. At 1 and 2-cm FOVs, those values were the same, therefore only one simulation was done with the optimum tube voltage; the second simulation was performed with the "typical" tube voltage of $80 \mathrm{kVp}$, which was the tube voltage most frequently used, according to the literature, to measure the detector presampling MTF. Overall, 40 detector presampling LSFs and corresponding MTFs were simulated.

The simulation parameters were the following. The number of samples in each simulated LSF was 512. This corresponded to 512 runs of the Monte Carlo code (each run with a slightly shifted, along the detector arm, $\mathrm{x}$-ray source position) to obtain one LSF. The amount of shifting (the LSF sampling distance) was equal to $1 / 16$ of the projected cell width. The number of photon histories in each run was 10,000. These values of the simulation parameters were chosen to ensure that the statistical error in the simulated detector presampling MTFs was always less than $0.3 \%$, whereas the aliasing and truncation errors were always below $0.5 \%$.

\subsection{Measurement of the detector presampling MTF}

The detector presampling MTF, as the descriptor of the pre-reconstruction spatial resolution of the experimental VRX CT scanner, was measured by the moving-slit method, which provided sufficiently-fine spatial sampling required for the LSF 
acquisition (Section 4.4.2). According to this method - and similarly to the modeling - a narrow slit was moved along the VRX detector, across a cell under study, and the signal (containing many time points) from that cell was recorded. This signal, after necessary processing, represented the measured LSF. The measured MTF was then computed and corrected for the effects of the focal spot and slit, to produce the detector presampling MTF.

The moving-slit method appeared to be the most suitable technique for measuring the detector presampling MTF in the experimental VRX CT scanner. Because the VRX detector was a 1D discrete detector, none of the methods developed for digital imaging systems with 2D detectors (i.e., with a stationary slanted slit or edge, as described in Section 4.4.2) could be used. Among the methods for systems employing 1D detectors (i.e., with a moving slit or edge), the use of a slit was preferable due to a higher SNR when measuring the high-frequency response (Sections 4.4.1 and 4.4.2). Also, as mentioned earlier, the ability to move the slit with an arbitrary small step (limited only by hardware) allowed sufficient LSF sampling, to avoid any aliasing errors.

\subsubsection{Experimental setup}

For the measurement of the detector presampling MTF, the experimental VRX CT scanner (Fig. 5.1) was slightly modified. The rotary table that supported an object during a CT scan was removed from the steel bench. Instead of the rotary table, an adjustable slit was installed between the slice-thickness collimator and the VRX detector. The slit was attached to a micropositioner that provided movement of the slit along the detector. 
To ensure accurate angular alignment of the VRX detector with respect to the $\mathrm{x}$ ray tube, the detector was placed on an acrylic plate that had a common pivotal point with the detector vertex (Fig. 5.7). The plate could be manually rotated with the 0.1-deg step in the \pm 2 -deg range from the detector centerline. The plate also had the pre-defined opening half-angles corresponding to the six selected FOVs (Table 5.3). During the alignment, the detector centerline was imitated by a thin wire tightened between the detector vertex and the point over the x-ray tube focal spot. The line representing the zero angle on the acrylic plate was then aligned with the wire, i.e., with the detector centerline. A custom-built slit $^{33}$ was used for the measurement (Fig. 5.7). The slit was formed by two 1.1-mm thick tungsten (W) jaws covered with 0.5-mm thick lead (Pb) plates on both sides. Each jaw was controlled by a separate micrometer (with $2-\mu \mathrm{m}$ graduation), to allow accurate adjustment of the slit width. The slit was placed on a National Aperture MM-4M-F-50 micropositioner (National Aperture Inc., Salem, NH), which provided the translational speed up to $6 \mathrm{~mm} / \mathrm{s}$, the positional accuracy of $\pm 1 \mu \mathrm{m}$, and the positional repeatability of $\pm 0.5 \mu \mathrm{m}$. The micropositioner was placed on a short aluminum bar, with a pivotal point on one side. This short bar was, in turn, placed on a long aluminum bar, also with a pivotal point on the same side. By rotating the short and long bars around their pivotal points, the slit was "rotated" around the vertical axis with coarse and fine steps, respectively. This allowed very accurate angular alignment of the slit with respect to $\mathrm{x}$ rays hitting a cell under study. The rotational step for the long bar (i.e., the fine alignment step) was $0.05 \mathrm{deg}$, with the total range of $\pm 2 \mathrm{deg}$.

To measure the detector presampling MTF, only one arm of the VRX detector was used (Fig. 5.7). The left arm, as viewed from the x-ray tube, was chosen. This arm 


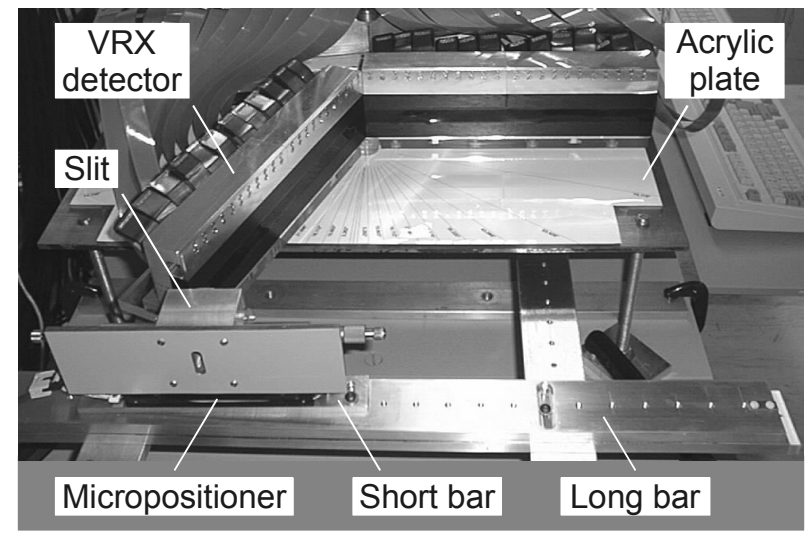

Figure 5.7. Slit and VRX detector setup for measuring the detector presampling MTF. An attenuator in front of the reference cells is not shown.

Source: Reprinted with permission. R. Melnyk and F. A. DiBianca, "Modeling and measurement of the detector presampling MTF of a variable resolution x-ray CT scanner," Med. Phys. 34, 1062-1075, 2007. 
was set at the opening half-angle corresponding to an FOV of interest. The other, right arm was set at the 90-deg angle with respect to the detector centerline. Several cells on the right arm were used as reference cells, allowing post-acquisition correction for variations in the x-ray tube output. To avoid signal saturation in the reference cells, an attenuator was placed in front of these cells. The attenuator was either a $1.0-\mathrm{mm}$ thick sheet of lead (used with high tube-voltage settings) or a 25.4-mm thick slab of acrylic (used with low tube-voltage settings).

As already mentioned, the slit was positioned between the slice-thickness collimator and the VRX detector. Reasonable attempts were made to position the slit as close to the detector as possible, to provide minimum system magnification. This was not always possible, however, because a few-millimeter gap had to be left between the slit and the detector edge to permit angular slit alignment, which involved movement of the slit in the source-detector direction. The slice-thickness collimator was placed at $70 \mathrm{~cm}$ from the x-ray tube focal spot, i.e., at the same distance used to compute the x-ray beam parameters of the experimental VRX CT scanner (Section 5.3.3). The X-ray tube, slicethickness collimator, slit, and VRX detector were also aligned in the vertical direction.

\subsubsection{Measurement procedure}

The measurement of the detector presampling MTF of the experimental VRX CT scanner included several steps. These can be divided into preliminary steps (air scan, slit alignment, and finding the effective slit gap), acquisition of actual data (i.e., LSF data used to compute the detector presampling MTF), and data processing (for all the preliminary-step and actual LSF data). 


\subsubsection{Preliminary steps}

First, the VRX detector was set at an FOV of interest (i.e., the left arm was set at the corresponding opening half-angle), the slit was removed, and an air-scan image was acquired. For the air scan, the opening of the slice-thickness collimator was $0.5 \mathrm{~mm}$, the tube voltage and current were $80 \mathrm{kVp}$ and $100 \mathrm{~mA}$, respectively, and the exposure time was $1 \mathrm{~s}$. The resulting image was processed to obtain a computer file with gains for the detector channels.

Then, the slit was put back in such a position that $\mathrm{x}$ rays passing through the slit would be incident on a cell under study during the slit movement. Identically to the modeling (Section 5.4.2), cells \#1,\#128, and \#256 were typically chosen as the cells under study. At 1 and 2-cm FOVs, however, only cell \#283 was considered because high system magnification for the cells closer to the detector vertex prohibited reliable measurement of the detector presampling MTF for those cells.

Cell \#1 (vertex cell) was chosen for the measurement because it was important to examine cells at the extreme positions in the VRX detector (all the other cells were intermediate) as the former cells would give the extreme MTF curves. The fact that there was a difference in the response of the vertex cell compared with the other cells, due to the vertex cell having no adjoining cell on one side making full contact as all the other cells did, was not a problem. This was because the measurement of the detector presampling MTF involved recording of the signal from the same cell, not from several adjacent cells. However, even if a series of adjacent cells were used, as when measuring the digital MTF, the vertex cell should still be included. In that case, there would just be two slightly lower points (from the two vertex cells) on the digital LSF, but this result 
would represent the actual situation in the VRX CT scanner.

While placing the slit in front of the cell under study, rough initial alignment of the slit was done to ensure an approximate 90-deg angle between the slit and the x rays incident on that cell. Then, more careful rotational alignment of the slit was performed. A series of LSF measurements was made by rotating the slit in increments of $0.05 \mathrm{deg}$. The integrals of the measured LSFs were computed and plotted versus the slit rotation angle. The angle with the maximum LSF integral corresponded to the optimum slit rotation angle. The slit was set at this angle and, thus, considered aligned with the cell under study.

For the slit alignment, the opening of the slice-thickness collimator and the slit gap were $3.8 \mathrm{~mm}$ and $10-20 \mu \mathrm{m}$, respectively. These settings ensured high x-ray intensity at the cell under study and, hence, a high SNR in the LSF measurements. The tube voltage, current, and exposure time were $80 \mathrm{kVp}, 100-250 \mathrm{~mA}$, and $4 \mathrm{~s}$, respectively.

After the slit was aligned, a procedure was performed to find the effective slit gap. The procedure included a series of LSF measurements with the decreasing slit gap. Several values of the slit gap in the range of 6-50 $\mu \mathrm{m}$ were used. The integrals of the measured LSFs were computed and plotted as points versus the slit-gap values. A linear fit to these points was then found. The abscissa of the intersection of this fit with the $x$ axis represented a "zero" slit gap. The effective slit gap was then computed by subtracting the "zero" slit gap from the actual slit gap, given by the micrometer readings. Because the "zero" slit gap was usually negative (i.e., x rays were passing even through the fully closed slit), the effective slit gap was typically greater than the actual slit gap. For this procedure, the slice-thickness collimator had the opening of $0.5 \mathrm{~mm}$, the tube 
voltage and current were $80 \mathrm{kVp}$ and 100-250 mA, respectively, and the exposure time was $4 \mathrm{~s}$.

\subsubsection{Acquisition of actual data}

After the slit was aligned and its effective gap was found, the actual LSF data used to compute the detector presampling MTF were acquired. The data were taken at three different tube voltages: (1) the optimum tube voltage for the "tissue" phantom, (2) the optimum tube voltage for the "bone" phantom, and (3) the "typical" tube voltage of $80 \mathrm{kVp}$, which was the most frequently used voltage, according to the literature, for measuring the detector presampling MTF. Ten LSF recordings were acquired at each tube voltage. This normally resulted in 30 LSF recordings for each cell under study at each selected FOV. The situation was slightly different at 1 and 2-cm FOVs, at which the optimum tube voltages for the "tissue" and "bone" phantoms were the same. Thus, at each of these two FOVs, only 20 LSF recordings were acquired for each cell under study.

When taking the actual LSF data, the opening of the slice-thickness collimator was $0.5 \mathrm{~mm}$. The exposure time was $4 \mathrm{~s}$. The tube current was the maximum allowable by the x-ray tube and generator for the chosen tube voltage and exposure time; this current ranged from 250 to $400 \mathrm{~mA}$. The translational speed of the micropositioner was chosen to provide the total length of each LSF approximately equal to 10 projected cell widths. Each LSF included about 4000 samples; this corresponded to the LSF sampling distance of roughly $1 / 400$ of the projected cell width. Such fine LSF sampling ensured aliasing-free MTF results. It also ensured that the blurring in the LSF signal due to the slit motion during the data acquisition was extremely small (around $0.25 \%$ of the projected 
cell width) and could be neglected. The actual (given by the micrometer readings) slit gap was typically $10 \mu \mathrm{m}$. For lower tube voltages at smaller FOVs, however, the slit gap was increased to provide an acceptable SNR. Thus, the $40-\mathrm{kVp}$ measurements at 1, 2, and 4cm FOVs were made with the slit gap of 20,14, and $12 \mu \mathrm{m}$, respectively. The increased slit gap did not affect the measurement results, which were already limited by the enlarged focal-spot size due to the focal-spot "blooming" (discussed later) at $40 \mathrm{kVp}$. Also, for $80 \mathrm{kVp}$ at $1-\mathrm{cm} \mathrm{FOV}$, the slit gap was $6 \mu \mathrm{m}$, to allow an accurate LSF measurement with still an adequate SNR.

\subsubsection{Data processing}

All LSF data - either actual, used to compute the detector presampling MTF, or auxiliary, used to align the slit and find the effective slit gap - were processed in the following way. An acquired image was first corrected for gain variations among the detector channels, using the channel gains from the computer file created during the air scan. The image was then corrected for the channel offsets, by subtracting the same cells' readings taken when $\mathrm{x}$ rays were off. Next, the image was corrected for time-dependent variations in the $\mathrm{x}$-ray tube output. This was done by dividing the image, sample by sample, by the average of the reference cells' signals. Finally, the measured LSF was extracted. For an auxiliary LSF (used to align the slit or find the effective slit gap), the extracted data were employed with no additional processing. For an actual LSF (used to compute the detector presampling MTF), the extracted LSF was further processed.

The further processing of an actual LSF included, first, an additional offset correction, to make sure the tails of the LSF were at the zero level. This was done by 
selecting two regions, one on each tail (where the LSF data stopped falling), fitting a single straight line through the data in both regions, and subtracting the fitted line from the original LSF. After this correction, the next step was to find the system magnification and the LSF sampling distance. Although these parameters could be computed from the measured distances (the source-vertex distance and the source-slit distance) and the experimental settings (the detector opening half-angle, the acquisition sampling time, and the micropositioner translational speed), the values would not be accurate enough due to difficulties in accurately measuring the focal-spot and slit positions. Therefore, the system magnification and the LSF sampling distance were determined using a slit "trace" in the acquired image (Fig. 5.8-a). This trace was formed by $\mathrm{x}$ rays passing through the slit as the slit was moving across the detector cells. From the trace, the cells traversed by the slit were first found (Fig. 5.8-b). Then, based on the known physical cell spacing and the detector opening half-angle, the distance $S$ (Fig. 5.8-c) covered by the slit trace as projected onto the cell-under-study plane (i.e., the plane orthogonal to the line passing through the x-ray source and the cell under study) was computed. This distance was divided by the number of samples to get the LSF sampling distance. The system magnification was then determined by dividing the sampling distance by the actual distance the slit moved between two samples. The values of the system magnification and the LSF sampling distance were averaged for all the LSF recordings for each cell under study at each selected FOV.

After the system magnification and the LSF sampling distance were found as part of the further LSF processing, the measured MTF was computed from the offsetcorrected LSF. This MTF was then corrected for the effects of the focal spot and slit, and 


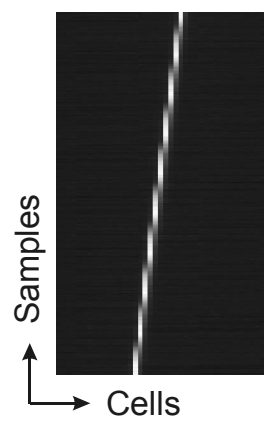

(a)

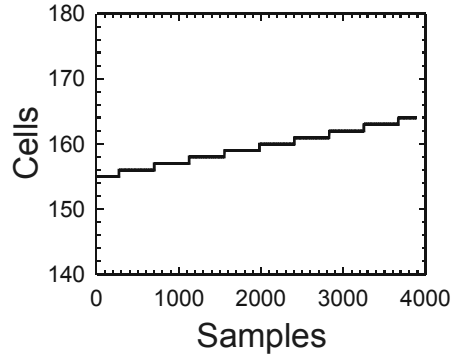

(b)

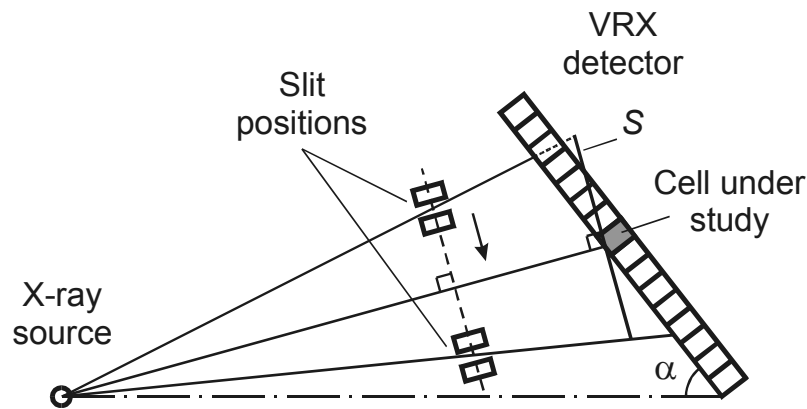

(c)

Figure 5.8. Method to determine the system magnification and the LSF sampling distance: (a) slit trace, (b) cells traversed by the slit, and (c) geometry used for the computation. In part (c), $\alpha$ - opening half-angle and $S$ - distance covered by the slit trace as projected onto the plane orthogonal to the line passing through the x-ray source and the cell under study.

Source: Reprinted with permission. R. Melnyk and F. A. DiBianca, "Modeling and measurement of the detector presampling MTF of a variable resolution X-ray CT scanner," Med. Phys. 34, 1062-1075, 2007. 
the final, detector presampling MTF was obtained. The MTF correction was made by dividing the measured MTF by the properly-scaled slit and focal-spot MTFs. The slit MTF was given by a sinc function corresponding to a rectangular LSF with the width equal to the effective slit gap. The focal-spot MTF was accurately measured using the same moving-slit method. For this measurement, the slit was placed close to the x-ray tube focal spot, to provide large system magnification (around 4.7). In addition, the right detector arm (as viewed from the x-ray tube), which was set at the 90-deg angle with respect to the detector centerline, was used for the measurement, to exclude any effects of the detector angulation. The focal-spot MTF was measured at $80 \mathrm{kVp}(250 \mathrm{~mA})$ and $40 \mathrm{kVp}(400 \mathrm{~mA})$, yielding the measured focal-spot size of 1.23 and $1.50 \mathrm{~mm}$, respectively. These values significantly exceeded the nominal focal-spot size of $0.6 \mathrm{~mm}$. Also, the $22 \%$ increase in the focal-spot size at $40 \mathrm{kVp}$ indicated the "blooming" of the focal spot, caused by repelling of electrons in the tube's electron beam at such a low voltage and a high current, due to the space charge effect. ${ }^{87}$ The $40-\mathrm{kVp}$ measured focalspot MTF was used to correct the 40-kVp detector presampling MTFs; all the other detector presampling MTFs were corrected with the $80-\mathrm{kVp}$ measured focal-spot MTF. The error introduced by such correction was believed to be negligible because the measured, not assumed, focal-spot MTF data were employed.

\subsubsection{Summary of the procedure}

After the detector presampling MTF was measured for one cell under study, the slit was repositioned for another cell under study at the same FOV, and the entire sequence - slit alignment, finding the effective slit gap, and actual MTF measurements - 
was repeated for the new cell. The same air-scan data were used for all the cells under study at the same FOV. After the detector presampling MTF was measured for all such cells at one FOV, the left arm of the VRX detector (as viewed from the X-ray tube) was set at the opening half-angle corresponding to another FOV, a new air-scan image was taken, and the entire measurement procedure was repeated.

To summarize, at each selected FOV, one air-scan image was acquired. For each cell under study at each selected FOV, a set of auxiliary LSF data (at different slit angles) was taken to align the slit, and another set of auxiliary LSF data (for different slit gaps) was taken to find the effective slit gap. Then, at each tube voltage for each cell under study at each selected FOV, 10 recordings of the actual LSF data were acquired. From these data, 10 measured LSFs were extracted, and 10 detector presampling MTFs were computed. From these 10 MTFs, the average MTF and the standard deviations were found. This average detector presampling MTF with its standard deviations at all the points represented the result of the measurement at one tube voltage for one cell under study at one selected FOV.

\subsection{Measurement of the scanner reconstruction MTF}

The reconstruction MTF, which characterized the post-reconstruction spatial resolution of the experimental VRX CT scanner, was measured by the LSF-phantom method (Section 4.4.3). According to this method, an acrylic phantom that contained a thin metal foil was imaged by the scanner, with the foil being orthogonal to the scan plane. The resulting data were reconstructed and adequately processed, producing an image in which the cross-section of the foil was represented by a segment of a blurred 
line. A profile across that segment corresponded, after necessary corrections, to the scanner reconstruction LSF, which was then converted to the reconstruction MTF by means of the Fourier transform.

The choice of the LSF-phantom method to measure the reconstruction MTF of the experimental VRX CT scanner was based on the following considerations (as per the description in Section 4.4.3). First, this method provided less noise than the PSF-phantom technique. Second, the LSF-phantom method was believed to yield more accurate assessment of the scanner reconstruction MTF at high spatial frequencies compared with the ESF-phantom technique. Finally, the use of a line object (approximated by the foil) to measure the reconstruction MTF was equivalent to the use of a line object (approximated by the slit) to measure the detector presampling MTF; such equivalence was thought to ensure more comparable results for the two components of the scanner spatial resolution.

\subsubsection{Experimental setup}

For the reconstruction MTF measurement, the experimental VRX CT scanner was used in its normal configuration, with the rotary table installed between the slicethickness collimator and the VRX detector. The rotary table provided rotation of an LSF phantom around its vertical axis. To avoid wobbling of the phantom during the rotation, the phantom was held on the top plate of the rotary table with the help of the four adjustable brackets.

The same acrylic plate that was inserted between the VRX detector and its support table when measuring the detector presampling MTF (Section 5.5.1) was employed in the current measurement as well. As before, the purpose of this plate was to 
allow accurate angular alignment of the VRX detector with respect to the x-ray tube. Also, the marks on the top surface of the plate for the six opening half-angles permitted accurate angulation of the detector arms according to the six selected FOVs (Table 5.3). Because the measurement of the scanner reconstruction MTF was done right after the measurement of the detector presampling MTF, and no changes were made to the arrangement of the VRX detector (except for the angulation) or the position of its support table, the detector was assumed to be still aligned with the $\mathrm{x}$-ray tube. Therefore, no additional alignment of the VRX detector was actually performed.

Due to the variable FOV of the experimental VRX CT scanner, not one but three LSF phantoms of different sizes were used to measure the reconstruction MTF (Fig. 5.9). These phantoms were built in house and had a construction similar to the AAPM design (Section 4.4.3). ${ }^{77}$ Each phantom was a disk of solid acrylic that sandwiched a thin copper $(\mathrm{Cu})$ foil between the two halves so that the foil was orthogonal to the disk base. The sizes of the phantoms were chosen in accordance with the selected FOVs. The largest phantom was $16 \mathrm{~cm}$ in diameter and contained a $76-\mu \mathrm{m}$ thick and $3-\mathrm{cm}$ wide foil. The foil dimensions were equal to those in the AAPM design. This "large" LSF phantom was used for the reconstruction MTF measurement at 16 and 32-cm FOVs. The medium-sized phantom was $4 \mathrm{~cm}$ in diameter and included a $25-\mu \mathrm{m}$ thick and $0.8-\mathrm{cm}$ wide foil. In this case, the foil dimensions were obtained by scaling, proportionally to the phantom size, the corresponding parameters of the large phantom and rounding up the foil thickness to a value that was available commercially. Such a "medium" LSF phantom was utilized to measure the scanner reconstruction MTF at 4 and 8-cm FOVs. The last, smallest phantom was only $1 \mathrm{~cm}$ in diameter and employed a $13-\mu \mathrm{m}$ thick and $0.2-\mathrm{cm}$ wide foil. Again, the 


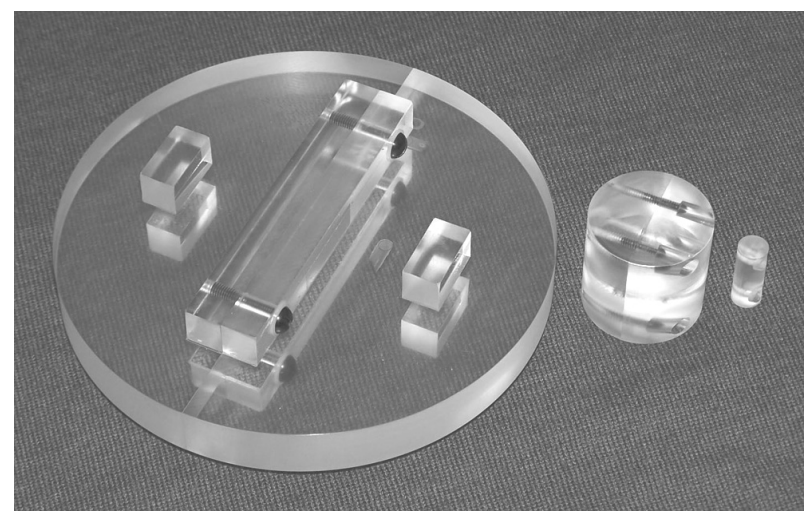

Figure 5.9. Large, medium, and small LSF phantoms for measuring the scanner reconstruction MTF. 
dimensions of the foil were scaled down from the large phantom, with the foil thickness selected based on the commercial availability. This "small" LSF phantom was intended for the measurement at 1 and 2-cm FOVs. The heights of the large, medium, and small phantoms were $4.5,3.9$, and $2.5 \mathrm{~cm}$, respectively. The foil height in the large and medium phantoms was $1.9 \mathrm{~cm}$, whereas this height in the small phantom was $0.8 \mathrm{~cm}$. Within each phantom, the foil was centered both horizontally and vertically. The summary of the parameters for the LSF phantoms used in the experimental VRX CT scanner is given in Table 5.5, along with the values for the AAPM LSF phantom.

When measuring the scanner reconstruction MTF, the arms of the VRX detector were placed symmetrically around the detector centerline, with each arm set at the opening half-angle corresponding to an FOV of interest. Because the 32 cells at the end of each arm were designated as reference cells (Section 5.3.2), care was taken not to block those cells, or at least the majority of them, from primary $\mathrm{x}$ rays with an LSF phantom during the measurement. This was achieved by proper lateral alignment of the rotary table on the steel bench as well as by accurate positioning of the phantom on the rotary table. The proper alignment included placing the rotary table so that its axis of rotation was slightly (by 1-2 mm) shifted from the detector centerline. Such a shift was found to provide better calibration and reconstruction results compared with placing the rotation axis exactly on the detector centerline. The accurate positioning of the phantom was done by visually centering the phantom on the top plate of the rotary table.

The slice-thickness collimator was kept at the same location $(70 \mathrm{~cm}$ from the $\mathrm{x}$ ray tube focal spot) at which it was set to measure the detector presampling MTF. Because the vertical positions of the x-ray tube, slice-thickness collimator, and VRX 
Table 5.5. Parameters of the LSF phantoms for the reconstruction MTF measurement.

\begin{tabular}{lcccc}
\hline $\begin{array}{c}\text { Phantom } \\
\text { description }\end{array}$ & $\begin{array}{c}\text { Phantom } \\
\text { diameter }(\mathrm{cm})\end{array}$ & $\begin{array}{c}\text { Foil } \\
\text { thickness }\end{array}$ & $\begin{array}{c}\text { Foil width } \\
(\mathrm{cm})\end{array}$ & $\begin{array}{c}\text { Foil height } \\
(\mathrm{cm})\end{array}$ \\
\hline AAPM phantom & 20 & $76 \mu \mathrm{m}(0.003 ”)$ & 3 & $1.5-2.5$ \\
VRX CT phantoms & 16 & $76 \mu \mathrm{m}(0.003 ”)$ & 3 & 1.9 \\
Large & 4 & $25 \mu \mathrm{m}(0.001 ”)$ & 0.8 & 1.9 \\
Medium & 1 & $13 \mu \mathrm{m}\left(0.0005^{\prime \prime}\right)$ & 0.2 & 0.8 \\
Small & & & & \\
\hline
\end{tabular}


detector did not change from the previous measurement, only additional vertical alignment of the rotary table was performed. Such alignment ensured that the vertical axis of an LSF phantom being imaged was orthogonal to the scan plane. The vertical alignment also ensured that the scan plane passed through the middle of the foil in the phantom.

\subsubsection{Measurement procedure}

The measurement of the reconstruction MTF of the experimental VRX CT scanner consisted of several steps. These included preliminary steps (air scan and scanner calibration), acquisition of actual data (i.e., phantom-image data used to compute the scanner reconstruction MTF), and data processing (for all the preliminary-step and actual phantom-image data).

\subsubsection{Preliminary steps}

Initially, the VRX detector was set at an FOV of interest (i.e., the detector arms were set at the corresponding opening half-angles), a proper tube voltage was selected, and an air-scan image was acquired with no LSF phantom placed on the rotary table. The tube voltage was chosen from the same three groups established before: (1) the optimum tube voltage for the "tissue" phantom (not to be confused with an LSF phantom), (2) the optimum tube voltage for the "bone" phantom, and (3) the "typical" tube voltage of $80 \mathrm{kVp}$. At each tube voltage, 10 air-scan images were taken and processed, to obtain 10 computer files with gains for the detector channels. For the air scans, the opening of the slice-thickness collimator was $0.5 \mathrm{~mm}$. The tube current ranged from 40 to $400 \mathrm{~mA}$, 
depending on the selected tube voltage. The exposure time was $1 \mathrm{~s}$.

The next step was scanner calibration. The purpose of the calibration was to accurately determine geometrical parameters needed for image reconstruction. These parameters included the opening half-angles for both detector arms and the locations of the two vertex cells (one on each arm) relative to the x-ray source and the rotation axis of the rotary table. The accuracy of the described parameters largely influenced the quality of reconstructed images. It was found that the values of these parameters taken directly from the experimental setup were not accurate enough, due to imperfections in machining the VRX detector and its support table as well as due to inevitable errors in aligning the scanner components. Therefore, these geometrical parameters were determined via a special calibration procedure, unique to the VRX CT scanner. In order to obtain the most accurate results, such calibration was repeated at each new FOV and tube voltage.

The previously developed procedure was utilized to calibrate the scanner. ${ }^{42}$ According to this procedure, a rigid steel pin was placed vertically on the rotary table, at a known distance from the rotation axis. The pin was scanned at the same settings that would be used to image an LSF phantom. The acquired sinogram contained a pin trajectory of a certain, non-uniform width. This trajectory was first converted to a mathematical (zero-width) curve by computing a centroid for each row (i.e., projection) of the pin sinogram. Then, the resulting curve was fitted with an analytical expression describing the formation of a pin sinogram for an ideal (zero-thickness) pin. The arguments of this expression corresponded to the six required geometrical parameters (three for each arm). The fitting was done by the multi-dimensional downhill simplex method (also known as the "amoeba" algorithm) ${ }^{88}$ The results of the fit, i.e., the accurate 
values of the six parameters, were saved into a so called "calibration" file, to be used for subsequent image reconstruction at the same FOV and tube voltage.

The scanner calibration was performed at the same three tube voltages selected for the air scan. At each tube voltage, 10 pin sinograms were acquired. These sinograms were separately analyzed by the calibration algorithm, to produce 10 calibration files. In all cases, the opening of the slice-thickness collimator was again $0.5 \mathrm{~mm}$. At the FOVs from 4 to $32 \mathrm{~cm}$, at which calibration data were collected, the pin thickness was $1 \mathrm{~mm}$. Calibration data were not taken at 1 and 2-cm FOVs, due to discovered difficulties with performing calibration at the small FOVs. The distance from the pin to the rotation axis of the rotary table at 4, 8, 16, and 32-cm FOVs was 2.19, 3.19, 7.19, and $15 \mathrm{~cm}$, respectively. The tube current was $40-400 \mathrm{~mA}$, depending on the selected FOV and tube voltage. The exposure time was $4 \mathrm{~s}$. The rotary table rotated counterclockwise with the angular speed of $100 \mathrm{deg} / \mathrm{s}$; this resulted in a slightly more than 360-deg rotation of the pin during an x-ray exposure, to allow some extra data in the pin sinogram.

\subsubsection{Acquisition of actual data}

After the scanner was calibrated, the actual image data used to compute the reconstruction MTF were acquired. For this purpose, the pin was removed, and an LSF phantom of the proper size was placed on the rotary table, with the foil oriented vertically (so that the foil was orthogonal to the scan plane). The phantom was scanned at exactly the same settings used for the calibration. The resulting sinogram was reconstructed employing a reconstruction algorithm developed earlier specifically for the experimental VRX CT scanner. ${ }^{41}$ This algorithm first converted the VRX CT sinogram to a uniformly- 
sampled sinogram, where all fan-angle increments were the same. The accurate conversion required precise knowledge of the locations of the detector arms; this information was read from a calibration file. Then, the data from the fan-beam geometry were transformed to a set of parallel-beam projections. The resulting sinogram in the parallel-beam space was finally reconstructed by the standard filtered-backprojection technique. ${ }^{2,7,89}$ The VRX CT reconstruction algorithm was implemented in MATLAB 7.0 (MathWorks Inc., Natick, MA), except for the filtered-backprojection routine, which was written in the $\mathrm{C}$ programming language and compiled using the LCC compiler included with MATLAB, to improve the algorithm's performance.

The sinogram of the LSF phantom was reconstructed with the 2048 by 2048 reconstruction matrix. This matrix 16 times exceeded, based on the number of pixels, the actual acquisition matrix ( 512 by 512 ) determined by the number of active cells in the VRX detector. Such a large reconstruction matrix was chosen to provide sufficiently-fine spatial sampling for the measured LSF, thereby avoiding any aliasing errors in the reconstruction MTF. Due to the much smaller-than-physical sampling in the reconstructed image, there was no need for the slight foil angulation during either phantom scanning or processing of the phantom image, as normally required by the LSFphantom method.

When acquiring a sinogram of the LSF phantom, the same three tube voltages the "tissue"-phantom tube voltage, the "bone"-phantom tube voltage, and the "typical" tube voltage - were chosen. At each tube voltage, 10 phantom sinograms were collected and separately reconstructed. For the reconstruction, the first sinogram was used with the first calibration file, the second sinogram was used with the second calibration file, and 
so on. This resulted in 10 reconstructed images of the LSF phantom. As before, the opening of the slice-thickness collimator was $0.5 \mathrm{~mm}$, the tube current was $40-400 \mathrm{~mA}$, and the exposure time was $4 \mathrm{~s}$. At 4 and 8-cm FOVs, the scanning was done with the medium (4-cm) phantom, whereas at 16 and 32-cm FOVs, the large $(16-\mathrm{cm})$ phantom was imaged. No phantom data were taken at 1 and 2-cm FOVs, as it would be impossible to reconstruct those data without calibration, which could not be performed correctly at those FOVs, as mentioned previously. The angular speed of the rotary table was again $100 \mathrm{deg} / \mathrm{s}$ counterclockwise, providing more than 360-deg of sinogram data for the given exposure time.

\subsubsection{Data processing}

Each pin or phantom sinogram underwent the following pre-processing before it was fed into the calibration or reconstruction algorithm, respectively. The raw sinogram was initially corrected for gain variations among the detector channels, utilizing the channel gains from a computer file created during the air scan. (Out of 10 acquired sinograms, either pin or phantom, and 10 created gain files, the first sinogram was used with the first file, the second sinogram was used with the second file, and so forth.) The sinogram was then corrected for the channel offsets, by subtracting the same cells' readings taken when $\mathrm{x}$ rays were off. At last, the sinogram was corrected for timedependent variations in the $\mathrm{x}$-ray tube output. This correction was done by dividing the sinogram, row by row, by the average of the reference cells' signals. After such preprocessing, the sinogram was used according to its content. The pin sinogram was analyzed by the calibration algorithm, to generate a calibration file. The phantom 
sinogram, on the other hand, was passed to the reconstruction algorithm, to obtain a cross-sectional image of the LSF phantom.

After each phantom image was reconstructed, it was processed in the following way. First, the edges of the phantom were located by thresholding (based on a difference in pixel intensities between the phantom and its background). This information was used to compute the phantom size (averaged over two orthogonal directions) in pixels. From that size and the known physical dimension of the phantom, the distance in millimeters between two pixels was determined; this was the sampling distance. Then, the position and direction of the foil in the phantom were found, respectively by thresholding and by fitting a straight line through the points representing above-threshold pixel intensities. The phantom image was next rotated so that the foil was aligned with the rows of the image pixel matrix. To avoid degradation of image quality during the rotation, the original image was resampled (via bilinear interpolation) to include four times more rows and columns, the resampled image was rotated, and the result was resampled back to the original size. After the rotation, the direction of the foil was detected again. If the angle between the foil and the rows of the image pixel matrix exceeded 0.1 deg, a slightly different threshold to find the foil was chosen, and the described procedure was repeated, starting with the foil detection in the original phantom image, until desired co-angularity was achieved.

From the rotated phantom image, 50 profiles across the foil were extracted; each profile was 256-pixel long and was centered on the foil. These profiles were averaged to produce the measured LSF. This LSF was additionally offset-corrected, to ensure that its tails were at the zero level. Such correction was made by selecting two regions, one on 
each tail (where the LSF data stopped falling), fitting a single straight line through the data in both regions, and subtracting the fitted line from the original LSF. After this correction, the measured LSF was converted to the corresponding MTF. The final, scanner reconstruction MTF was obtained by correcting the measured MTF for the foil thickness. This was done by dividing the measured MTF by a properly-scaled sinc function representing the foil MTF.

\subsubsection{Summary of the procedure}

After the reconstruction MTF of the experimental VRX CT scanner was measured for all three tube voltages at one FOV, the arms of the VRX detector were set at the opening half-angles corresponding to another FOV, and the entire measurement procedure was repeated. Because all the three main steps of this procedure - air scan, scanner calibration, and LSF-phantom scan - were performed at each new FOV and tube voltage, every phantom image was produced using its "own" air-scan and calibration data. Moreover, these two types of data were collected at exactly the same other settings - the opening of the slice-thickness collimator and the tube current - as the phantomimage data. Such consistency was followed to ensure high-quality measurement results.

In summary, for each tube voltage at each selected FOV, 10 air-scan images, 10 pin sinograms, and 10 phantom sinograms were acquired. The air-scan images were processed to create 10 files with channel gains. These files were utilized during the preprocessing of both the pin and phantom sinograms. Specifically, gain file \#1 was used with pin sinogram $\# 1$ and phantom sinogram \#1, gain file \#2 was used with pin sinogram \#2 and phantom sinogram \#2, and so on. The pre-processed pin sinograms were analyzed 
to generate 10 calibration files. These calibration files were employed during the reconstruction of the phantom sinograms. Like before, calibration file \#1 was used with phantom sinogram \#1, calibration file \#2 was used with phantom sinogram \#2, and so forth. The pre-processed phantom sinograms were thus reconstructed producing 10 crosssectional images of the LSF phantom. From these images, after additional manipulations, 10 measured LSFs (each averaged over 50 LSF samples) were obtained, and 10 scanner reconstruction MTFs were computed. From these 10 MTFs, the average MTF and the standard deviations were found. This average scanner reconstruction MTF with its standard deviations at all the points represented the result of the measurement for one tube voltage at one selected FOV.

Some difficulties encountered while measuring the reconstruction MTF of the experimental VRX CT scanner should be noted here. All necessary air-scan, calibration, and phantom-scan data were collected at 4, 8, 16, and 32-cm FOVs. However, due to discovered limitations of the utilized calibration algorithm, only the pin sinograms at 8 , 16, and 32-cm FOVs could be successfully processed. Despite successful processing of the calibration data at $32-\mathrm{cm}$ FOV, accurate reconstruction of the phantom sinograms at this FOV could not be performed. Therefore, only the phantom-scan data taken at 8 and 16-cm FOVs were reconstructed and further processed. As a result, the measured scanner reconstruction MTF was determined only at these two FOVs. Because the problem with processing the pin sinograms at 4-cm FOV was believed to stem from a larger impact of the machining and alignment errors as well as from a lower SNR, and both these factors were attributed to the reduced FOV, it was decided not to repeat the measurement at the 
smaller FOVs (1 and $2 \mathrm{~cm})$, as successful scanner calibration at those FOVs would be even less possible with the employed version of the calibration algorithm. 


\section{CHAPTER 6. RESULTS AND DISCUSSION*}

This chapter presents the results of the current study on the evaluation of spatial resolution in the experimental VRX CT scanner - specifically, the modeling and measurement results for the detector presampling MTF and the measurement results for the scanner reconstruction MTF. The chapter also discusses the implications and importance of the obtained results and provides suggestions regarding the x-ray tube parameters that would have eliminated some of the problems faced in the study.

\subsection{Modeling of the detector presampling MTF}

The detector presampling MTF, which represented the pre-reconstruction spatial resolution of the experimental VRX CT scanner, was modeled by the Monte Carlo simulation, as described in Section 5.4. Before considering the modeling MTF results, it was helpful to examine first the corresponding LSF. A sample detector presampling LSF simulated at the "tissue"-phantom tube voltage for cell \#256 at 8-cm FOV is shown in Fig. 6.1-a. The LSF was asymmetrical, due to the angulation of the VRX detector. The tail directed toward the end of the detector arm (left tail in Fig. 6.1-a) was higher than the tail (right tail) directed toward the detector vertex. A theoretical comparison of symmetrical and asymmetrical LSFs with the same FWHM revealed that the LSF asymmetry did not significantly affect the MTF cutoff frequency but only lowered the MTF curve. A small "plateau" on the left side of the peak of the modeled detector

\footnotetext{
* Sections 6.1, 6.2, and 6.4 of this chapter adapted with permission. R. Melnyk and F. A. DiBianca, "Modeling and measurement of the detector presampling MTF of a variable resolution x-ray CT scanner," Med. Phys. 34, 1062-1075, 2007.
} 


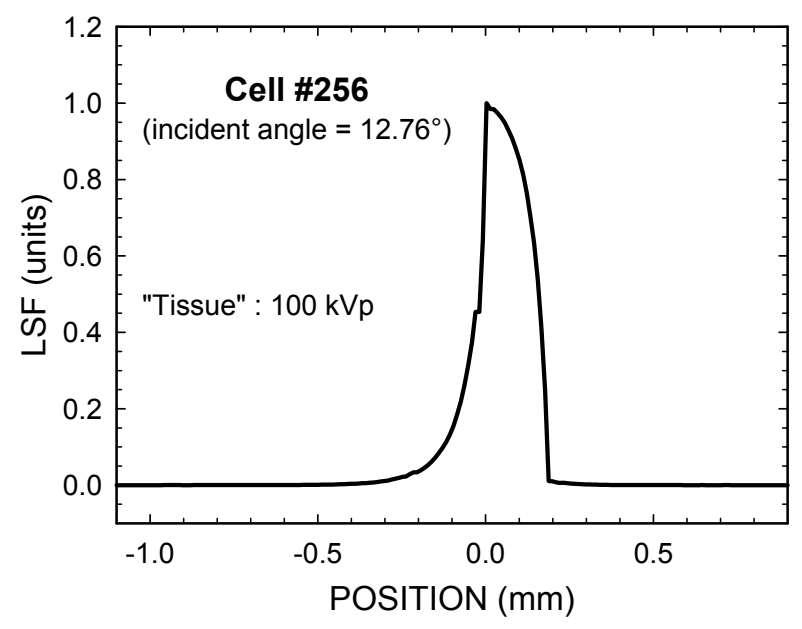

(a)

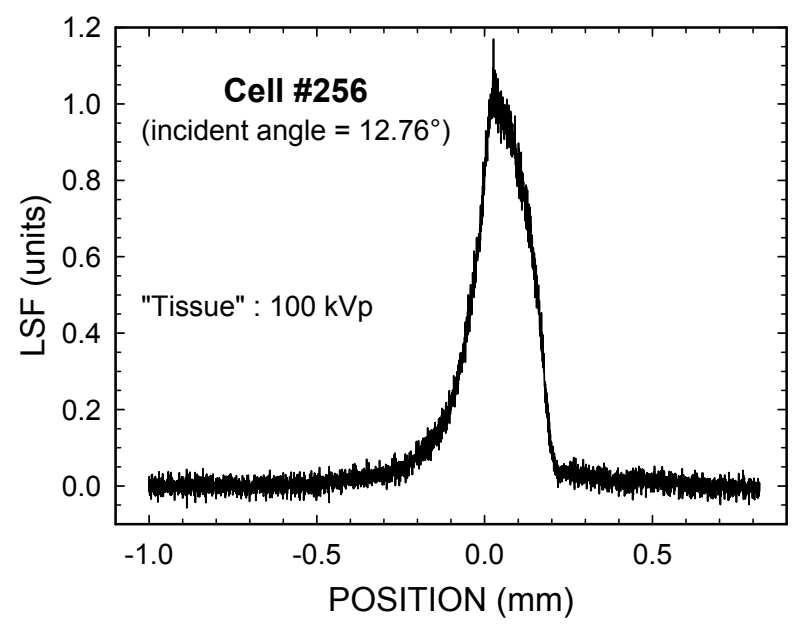

(b)

Figure 6.1. Sample modeled and measured detector response at 8-cm FOV: (a) modeled detector presampling LSF and (b) measured LSF used to compute the measured detector presampling MTF.

Source: Reprinted with permission. R. Melnyk and F. A. DiBianca, "Modeling and measurement of the detector presampling MTF of a variable resolution x-ray CT scanner," Med. Phys. 34, 1062-1075, 2007. 
presampling LSF (around $-0.03 \mathrm{~mm}$ ) corresponded to those $\mathrm{x}$-ray beam positions, as the beam was moving along the detector, where $\mathrm{x}$ rays were passing only through the reflective paint before entering the cell under study. Because of zero attenuation in the gap corresponding to the reflective-paint coating (this coating was not modeled, as described in Section 5.4.1), there was very little change in the energy deposited in the cell, and, therefore, the LSF was almost flat over the region equal to the projected width of the reflective paint.

The results of the Monte Carlo simulation of the detector presampling MTF of the experimental VRX CT scanner are shown in Figs. 6.2-6.7 as solid and dashed lines with no symbols. For comparison, these figures also include the measurement results (lines with symbols) as well as the ideal detector presampling MTF (dotted lines with no symbols). The ideal MTF was just a sinc function representing a rectangular LSF of the width equal to the projected cell width. All the MTFs were given in the detector (image) plane, which in the angulated VRX detector corresponded to the specific cell-under-study plane (i.e., the plane that crossed the front surface of the cell under study and was orthogonal to the line passing through the x-ray source and that cell). From the graphs, three important observations were made regarding the results of the modeling.

First, at all the FOVs, except for 1 and $2 \mathrm{~cm}$, the MTF curves at the "tissue"phantom tube voltage were always lower than the MTF curves at the "bone"-phantom tube voltage. This was because the "tissue"-phantom tube voltage was always higher than the "bone"-phantom tube voltage at those FOVs, and the higher tube voltage resulted in more cell-to-cell x-ray penetration, which degraded the detector presampling MTF. The discrepancy between the "tissue" and the "bone" MTF curves increased as the difference 

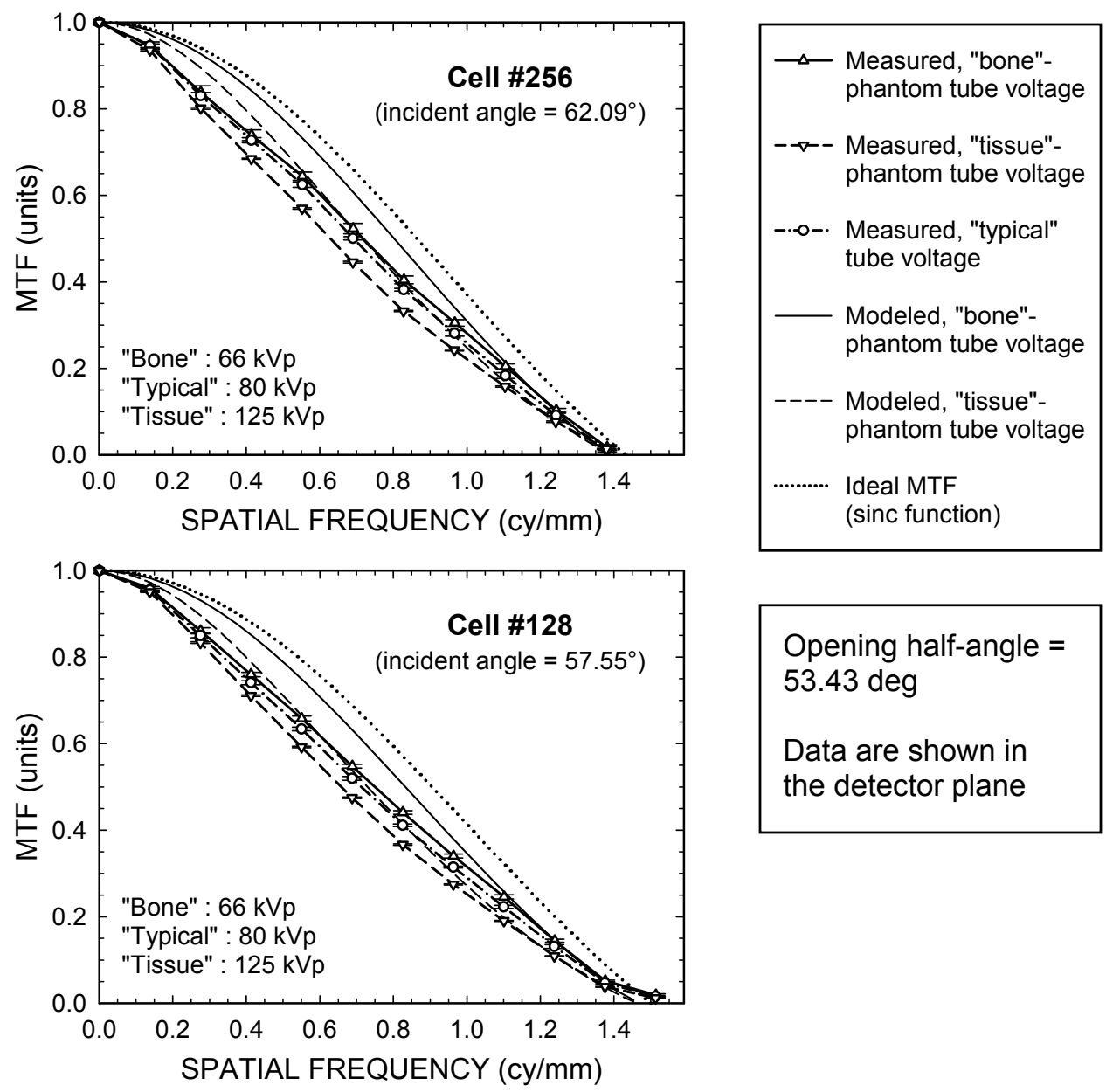

Opening half-angle $=$ $53.43 \mathrm{deg}$

Data are shown in the detector plane

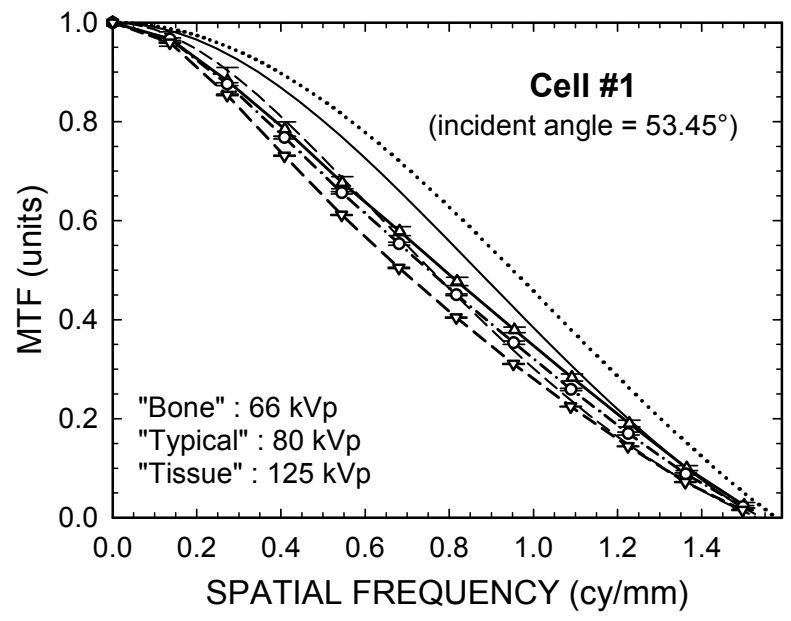

Figure 6.2. Modeled and measured detector presampling MTF at 32-cm FOV.

Source: Adapted with permission. R. Melnyk and F. A. DiBianca, "Modeling and measurement of the detector presampling MTF of a variable resolution x-ray CT scanner," Med. Phys. 34, 1062-1075, 2007. 

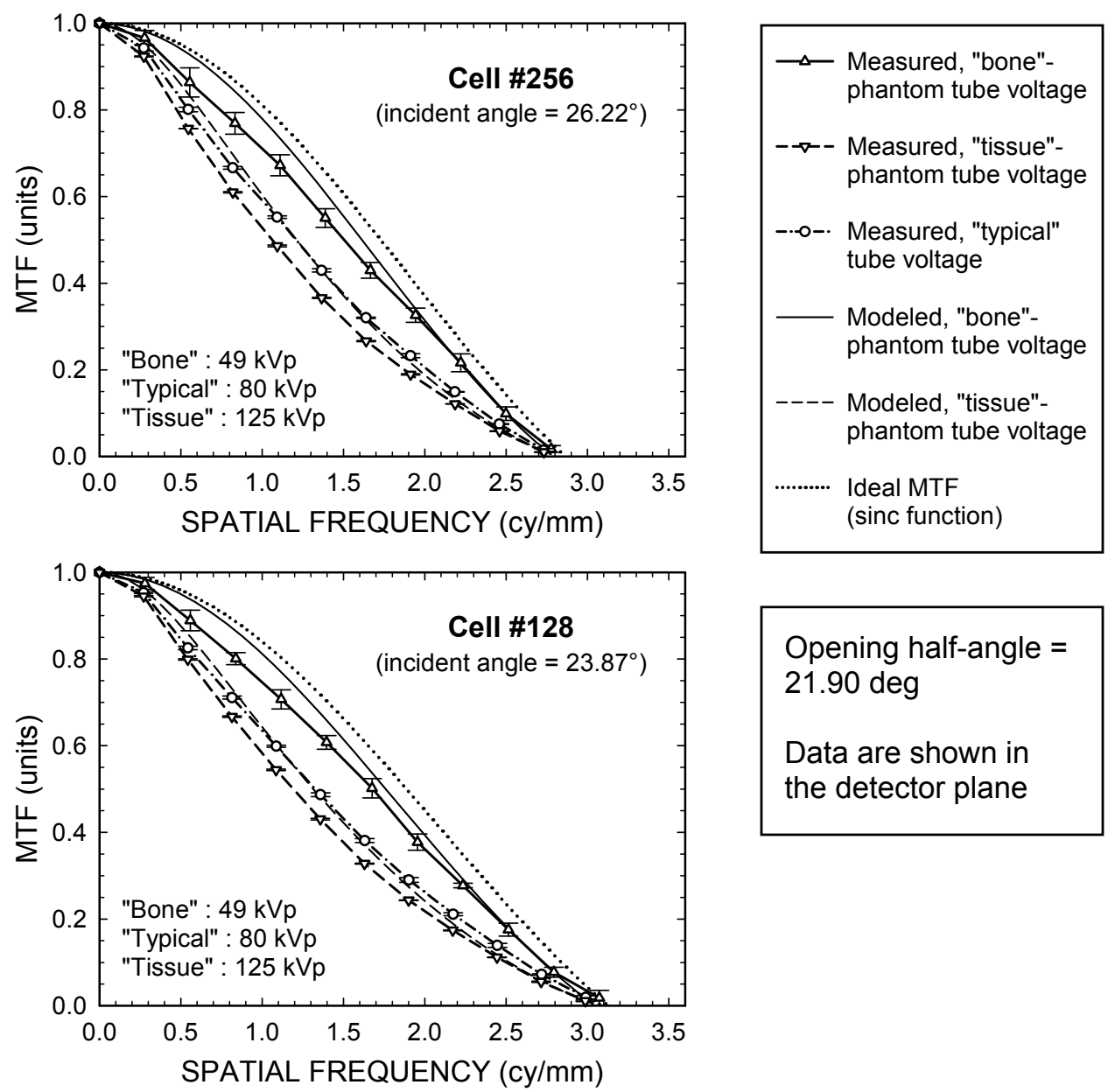

Opening half-angle $=$ $21.90 \mathrm{deg}$

Data are shown in the detector plane

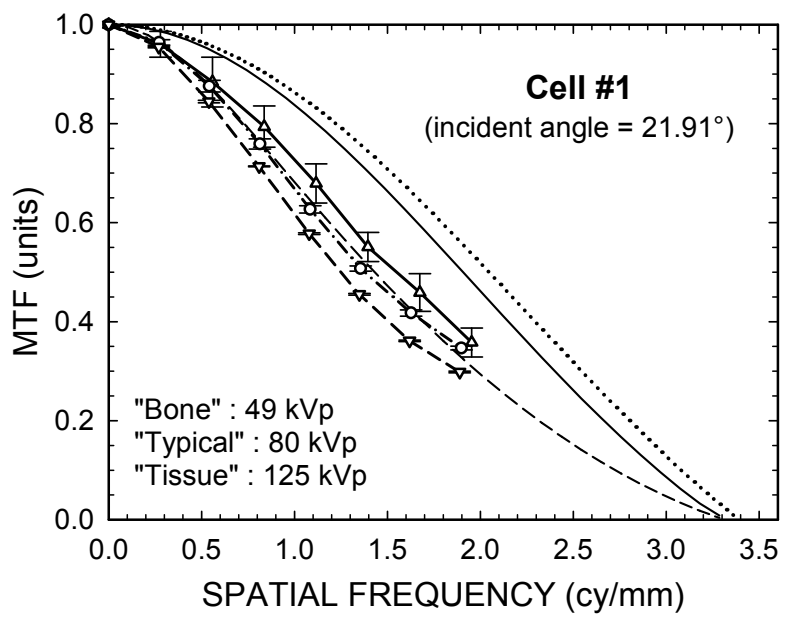

Figure 6.3. Modeled and measured detector presampling MTF at 16-cm FOV.

Source: Adapted with permission. R. Melnyk and F. A. DiBianca, "Modeling and measurement of the detector presampling MTF of a variable resolution x-ray CT scanner," Med. Phys. 34, 1062-1075, 2007. 

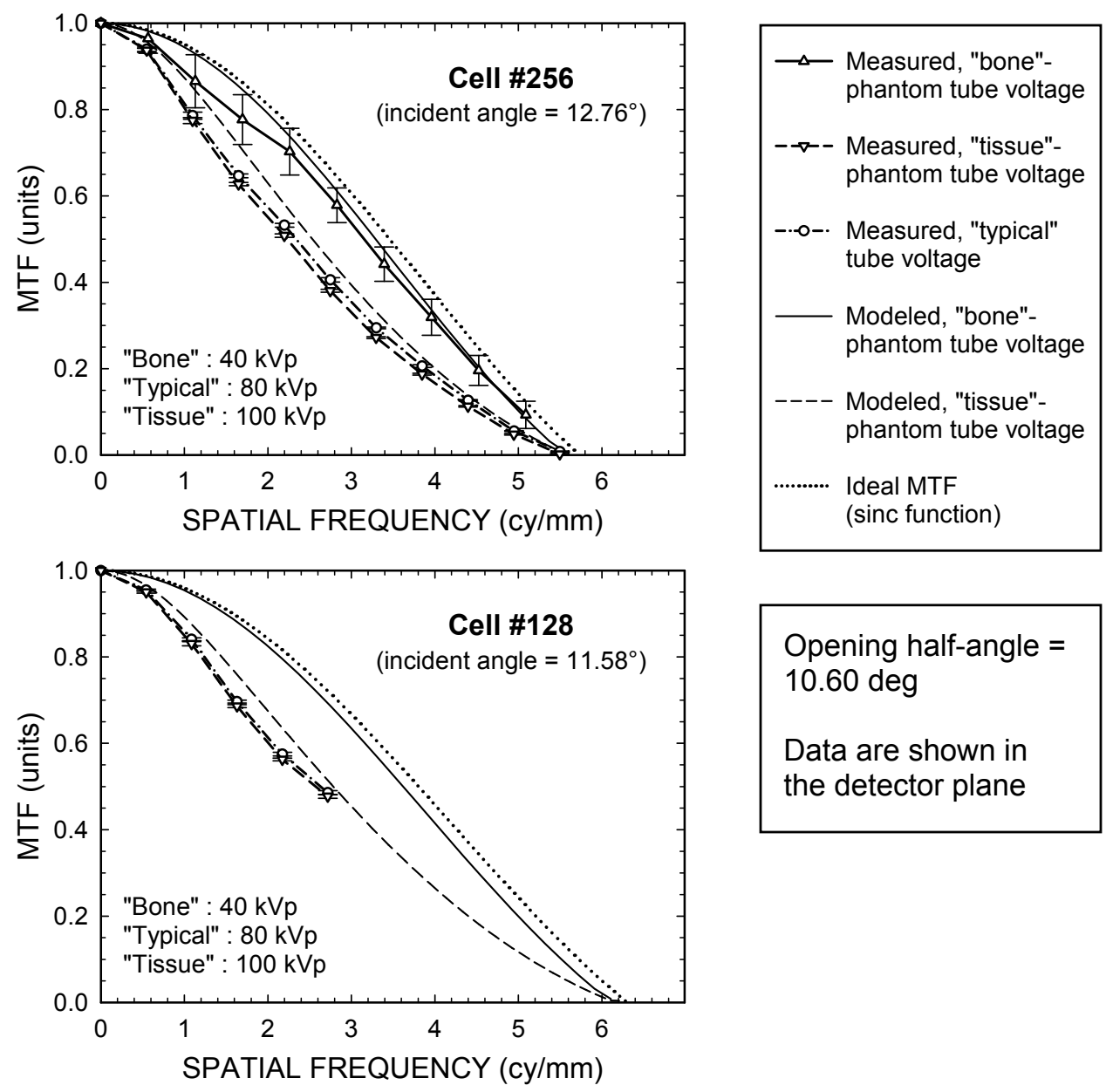

Opening half-angle $=$ $10.60 \mathrm{deg}$

Data are shown in the detector plane

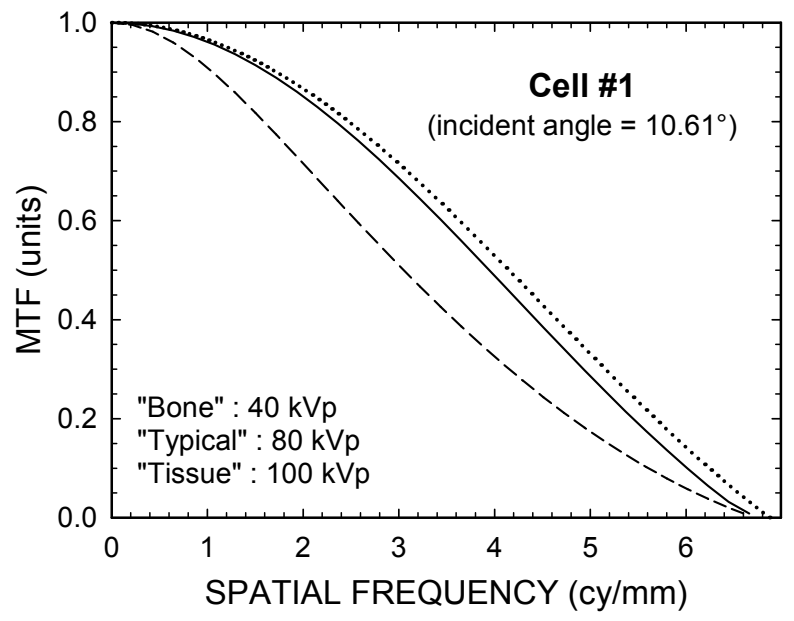

Figure 6.4. Modeled and measured detector presampling MTF at 8-cm FOV.

Source: Adapted with permission. R. Melnyk and F. A. DiBianca, "Modeling and measurement of the detector presampling MTF of a variable resolution x-ray CT scanner," Med. Phys. 34, 1062-1075, 2007. 

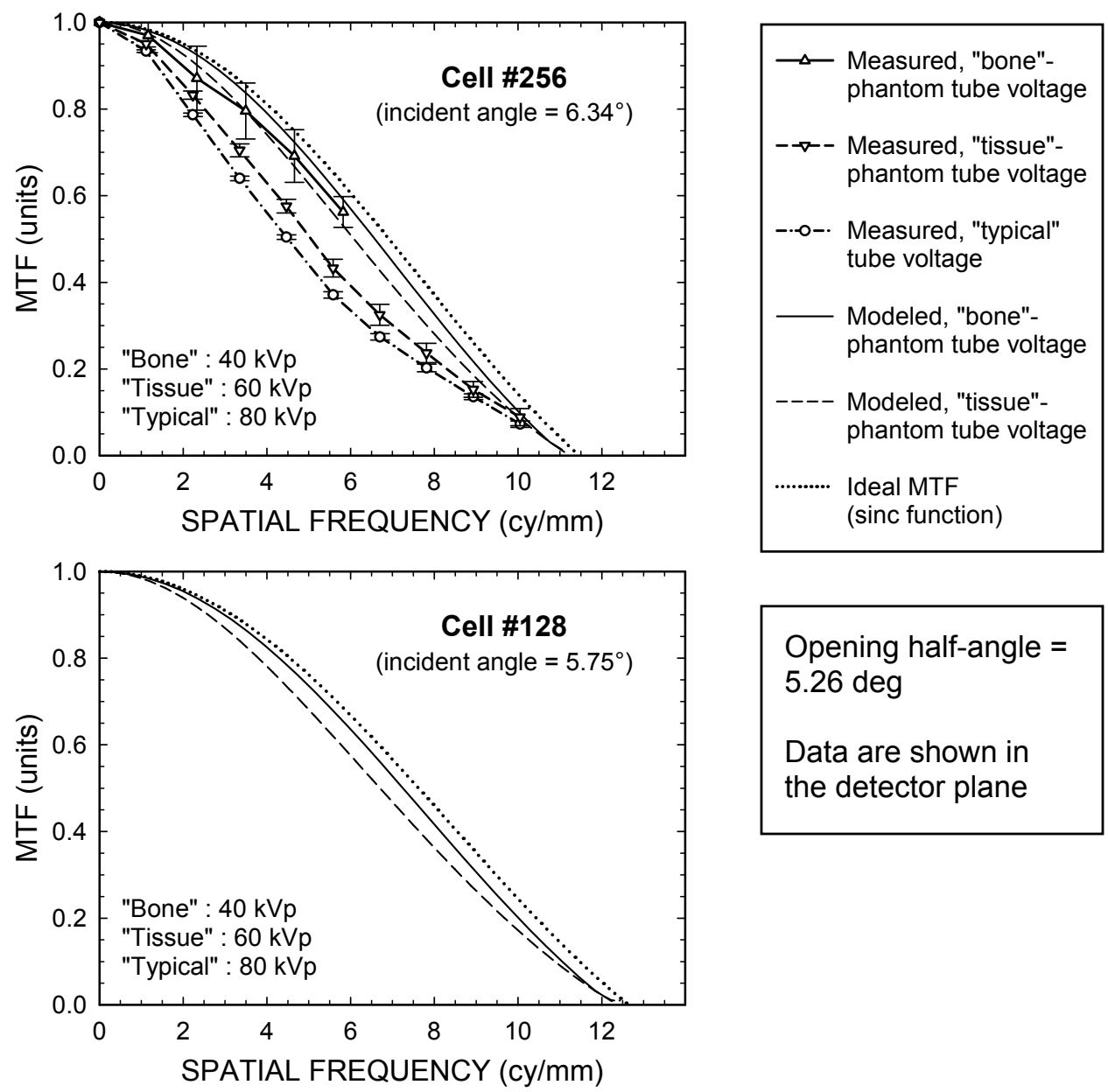

Opening half-angle $=$

5.26 deg

Data are shown in the detector plane

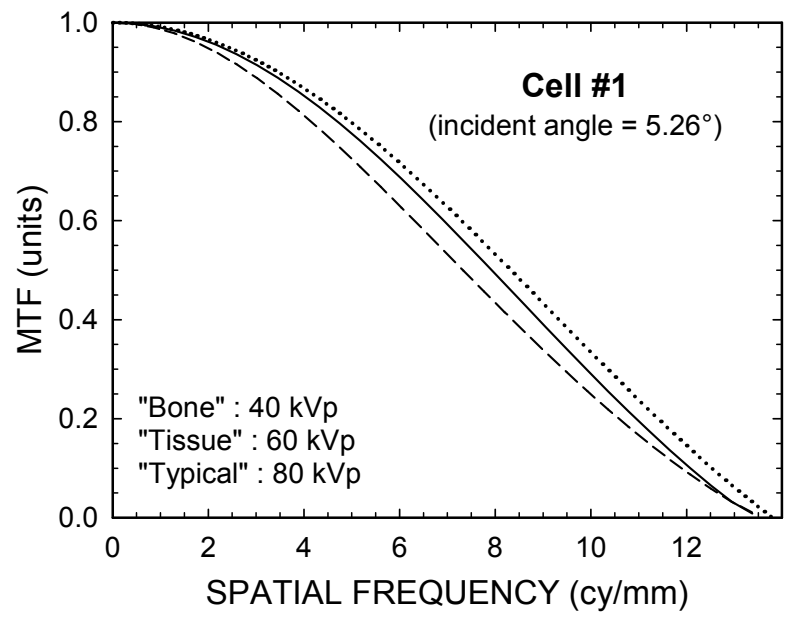

Figure 6.5. Modeled and measured detector presampling MTF at 4-cm FOV.

Source: Adapted with permission. R. Melnyk and F. A. DiBianca, "Modeling and measurement of the detector presampling MTF of a variable resolution x-ray CT scanner," Med. Phys. 34, 1062-1075, 2007. 

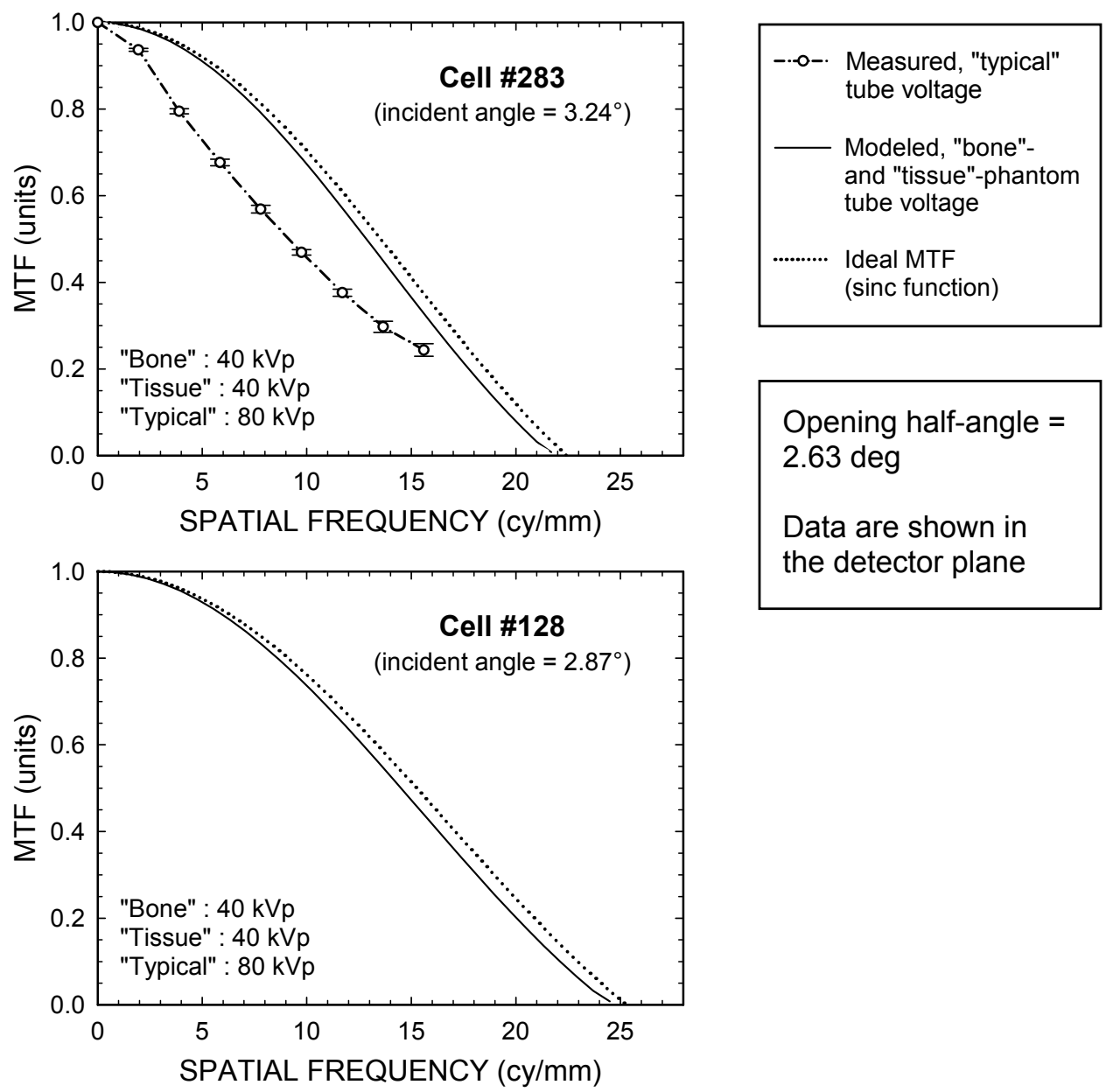

Opening half-angle $=$ $2.63 \mathrm{deg}$

Data are shown in the detector plane

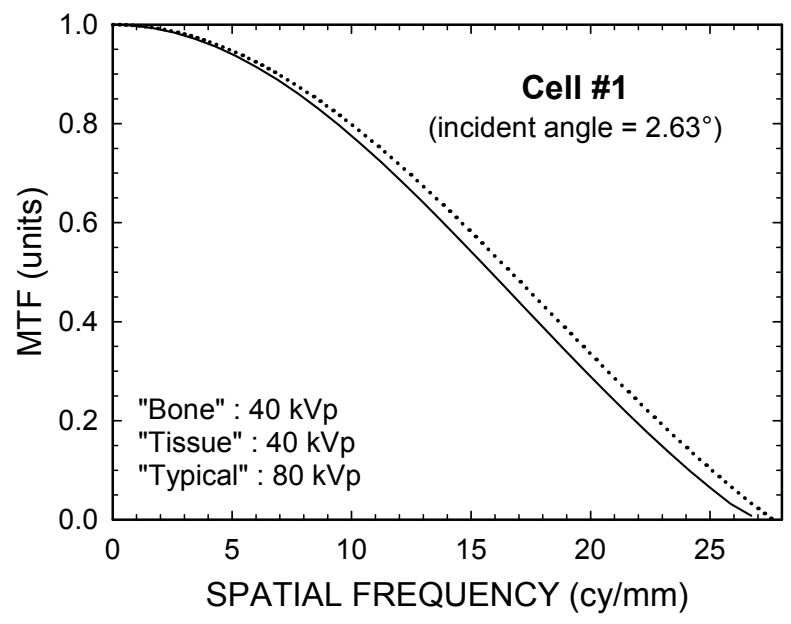

Figure 6.6. Modeled and measured detector presampling MTF at 2-cm FOV.

Source: Adapted with permission. R. Melnyk and F. A. DiBianca, "Modeling and measurement of the detector presampling MTF of a variable resolution x-ray CT scanner," Med. Phys. 34, 1062-1075, 2007. 

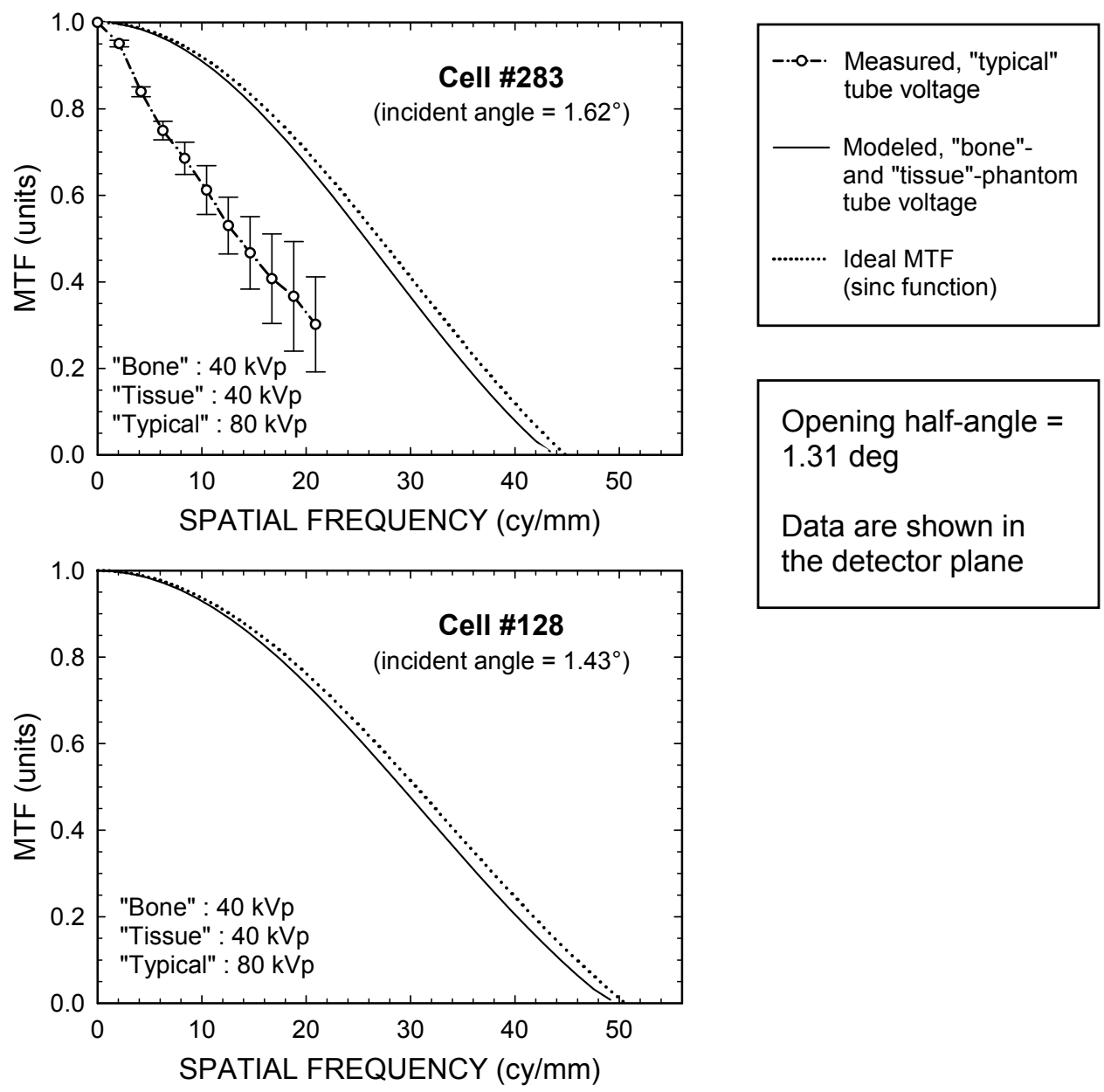

Opening half-angle $=$ $1.31 \mathrm{deg}$

Data are shown in the detector plane

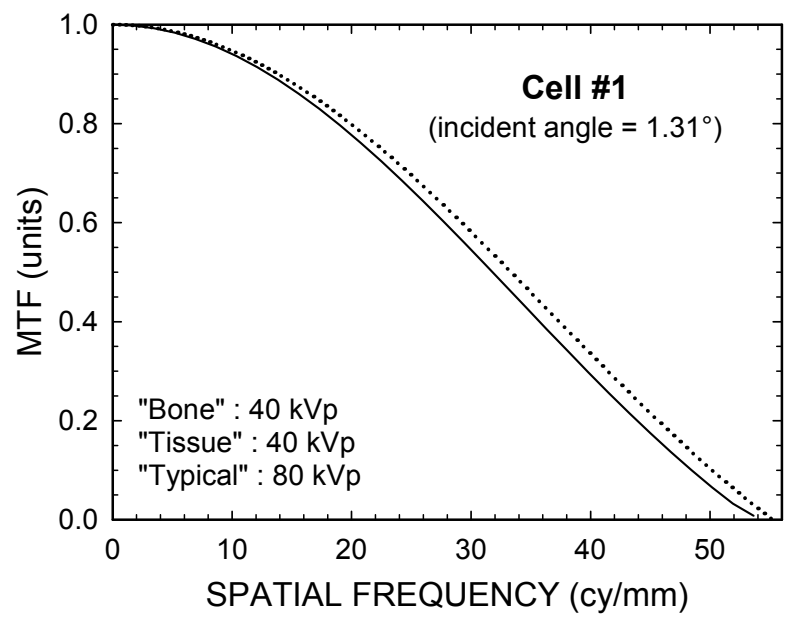

Figure 6.7. Modeled and measured detector presampling MTF at 1-cm FOV.

Source: Adapted with permission. R. Melnyk and F. A. DiBianca, "Modeling and measurement of the detector presampling MTF of a variable resolution x-ray CT scanner," Med. Phys. 34, 1062-1075, 2007. 
between the corresponding tube voltages became larger. At 1 and 2-cm FOVs, the "tissue"- and "bone"-phantom tube voltages were the same, producing identical MTF curves.

Second, despite the differences in the MTF curves, the MTF cutoff frequencies at the "tissue"- and "bone"-phantom tube voltages were very close. This was consistent with the idea that the limiting spatial resolution of the VRX detector should only weakly depend on the energy of the x-ray beam. Also, the simulated MTF cutoff frequencies were within approximately $3 \%$ of the ideal cutoff frequencies.

Third, as the FOV decreased from 32 to $1 \mathrm{~cm}$, the MTF cutoff frequency increased from $1.39 \mathrm{cy} / \mathrm{mm}$ (cell \#256) to $43.38 \mathrm{cy} / \mathrm{mm}$ (cell \#283). For the vertex cell (\#1), the MTF cutoff frequency increased from 1.53 to $53.64 \mathrm{cy} / \mathrm{mm}$. These important modeling results clearly demonstrated the increase in the spatial resolution of the VRX detector with its angulation. Also, at each FOV, except for $32 \mathrm{~cm}$, the MTF cutoff frequency increased by approximately $20 \%$ as one moved from the last active cell (\#256) to the vertex cell (\#1); this increase was only $10 \%$ at $32-\mathrm{cm} \mathrm{FOV.} \mathrm{To} \mathrm{summarize,} \mathrm{the}$ maximum MTF cutoff frequency predicted by the modeling was in the range of 43$54 \mathrm{cy} / \mathrm{mm}$; this frequency corresponded to the smallest selected FOV $(1 \mathrm{~cm})$.

The Monte Carlo simulation was also used to show the effect of the x-ray beam quality on the detector presampling MTF of the experimental VRX CT scanner (Fig. 6.8). It was apparent that at the incident angle of $11.58 \mathrm{deg}$ (for the middle active cell at $8-\mathrm{cm}$ FOV), increasing the beam energy from 29 to $69 \mathrm{keV}$ (by raising the tube voltage from 40 to $140 \mathrm{kVp}$ and simultaneously increasing the external aluminum filtration from 0 to $5 \mathrm{~mm}$ ) lowered the spatial frequency by as much as $35 \%$ for the MTF value of 0.5 


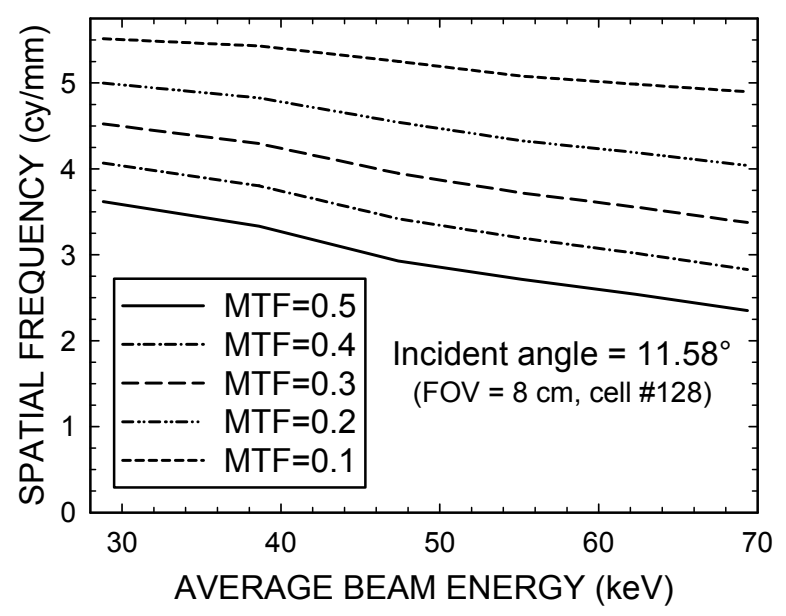

(a)

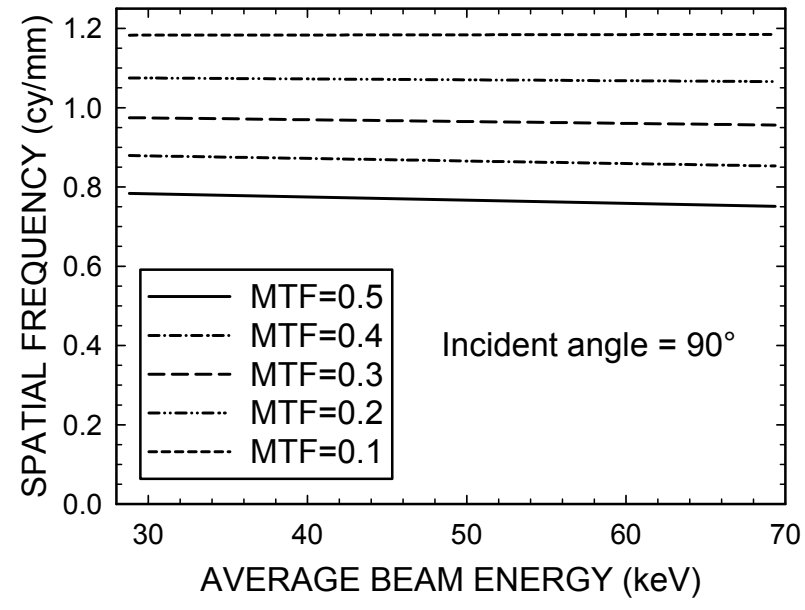

(b)

Figure 6.8. Modeled spatial resolution of the VRX detector vs. beam quality: (a) at the 11.58-deg incident angle and (b) at the 90-deg incident angle.

Source: Reprinted with permission. R. Melnyk and F. A. DiBianca, "Modeling and measurement of the detector presampling MTF of a variable resolution x-ray CT scanner," Med. Phys. 34, 1062-1075, 2007. 
(Fig. 6.8-a). But, as already demonstrated (Figs. 6.2-6.7), "hardening" of the x-ray beam did not significantly affect the MTF cutoff frequency. Although not generally appreciated, increasing the beam energy lowers the MTF even at normal (90-deg) incidence for all x-ray detectors, because of scattered x-ray and electron range effects. For the VRX detector at normal incidence (Fig. 6.8-b), the spatial frequency was reduced by $4 \%$ for the MTF value of 0.5 as the beam energy increased from 29 to $69 \mathrm{keV}$.

\subsection{Measurement of the detector presampling MTF}

The detector presampling MTF, as the descriptor of the pre-reconstruction spatial resolution of the experimental VRX CT scanner, was measured by the moving-slit method (discussed in detail in Section 5.5). Similarly to the modeling, examination of the measurement MTF results was preceded by taking a look at the involved LSF. A sample measured LSF that was used to compute the detector presampling MTF is shown in Fig. 6.1-b. This LSF was acquired at the "tissue"-phantom tube voltage for cell \#256 at 8$\mathrm{cm}$ FOV. Because exactly the same settings were chosen to model the detector presampling LSF given in Fig. 6.1-a, the two LSFs could be compared. As in the case of the modeled data, the measured LSF was asymmetrical, due to the detector angulation. The LSF asymmetry, however, did not greatly affect the MTF cutoff frequency but only degraded the MTF curve, as discussed previously. The small "plateau" present on the left side of the peak in the modeled detector presampling LSF was less evident in the measured data because of non-zero attenuation in the actual reflective-paint coating; this attenuation caused larger change in the energy deposited in the cell under study over the projected width of the reflective paint. Also, the measured LSF, when corrected for the 
slit and focal-spot effects, agreed well with the modeled detector presampling LSF.

The results of the measurement of the detector presampling MTF of the experimental VRX CT scanner by the moving-slit method are shown in Figs. 6.2-6.7 as solid, dashed, and dash-dotted lines with symbols. As mentioned before, these figures also include the modeling results (solid and dashed lines with no symbols) as well as the ideal detector presampling MTF (dotted lines with no symbols). As a reminder, all the MTFs were given in the detector plane. Good quality MTF measurements were obtained for all the cells under study at 16 and 32-cm FOVs. At the smaller FOVs $(1-8 \mathrm{~cm})$, reliable MTF measurements could not be made for the cells close to the detector vertex (i.e., the cells with increased system magnification) because the cutoff frequency of the projected focal spot fell below the detector cutoff frequency for those cells. Therefore, the detector presampling MTF was measured only for cells \#128 and \#256 at 8-cm FOV, cell \#256 at 4-cm FOV, and cell \#283 at 2 and 1-cm FOVs. All the measured MTF curves were relatively smooth despite the noisy LSF data because, first, each curve represented an average of 10 MTFs computed from individual LSFs and, second, the frequency of the noise was typically far above the MTF cutoff frequency.

Again, several important observations were made based on the results. First, similarly to the modeled data, the measured MTF curves at the higher (usually "tissue"phantom) tube voltage were lower than the measured MTF curves at the lower ("bone"phantom) tube voltage, due to increased cell-to-cell x-ray penetration at the higher tube voltage. Also, the error bars were larger at the lower ("bone"-phantom) tube voltage, because the SNR in each low-voltage LSF recording was lower, but the same number of LSF recordings (10) was made to obtain the averaged MTF at the low and high tube 
voltages.

Second, the measured MTF cutoff frequencies at the "tissue"-phantom, "bone"phantom, and "typical" tube voltages were very close at the large FOVs $(16-32 \mathrm{~cm}$; except for cell \#1 at 16-cm FOV). At the small FOVs $(1-8 \mathrm{~cm}$; also for cell \#1 at 16-cm FOV), those cutoff frequencies were difficult to compare because the MTF curves in most cases were terminated at spatial frequencies below the expected cutoff frequencies, to provide the MTF data not degraded by the projected focal spot. Specifically, in each case when the estimated focal-spot cutoff frequency was close to or slightly below the detector cutoff frequency, the detector presampling MTF was terminated at a data point with the spatial frequency not exceeding $75 \%$ of the corresponding focal-spot cutoff frequency. The observed fact that the $40-\mathrm{kVp}$ MTF curves were terminated at much lower spatial frequencies or could not be measured at all was attributed to the increase in the focal-spot size due to the "blooming" effect, described previously.

Third, the measured MTF curves were always lower than their modeled counterparts. The difference between the corresponding measured and the modeled MTF curves was usually larger at the low tube voltage. The lower measured MTF curves could be explained, in the author's opinion, by physical phenomena present in the actual experimental VRX CT scanner but not included in the Monte Carlo simulation. Those were the following phenomena: (1) deviations between the true and the modeled detector and slit; (2) cell-to-cell non-uniformities, both geometrical and electronic; (3) cell-to-cell optical cross-talk via the optical epoxy that attached the scintillator crystals to the photodiodes; (4) x-ray scatter from the various detector components, the rotary table, and the nearby structures; (5) differences between the true and the modeled scanner 
geometry; (6) electronic noise, cross-talk, and other imperfections; (7) system noise, especially at small angles; and (8) focal-spot motion (target wobble, etc.).

Fourth, despite the explained discrepancies between the measured and the modeled MTF curves, their cutoff frequencies agreed well in all the cases where the measured values could be correctly estimated. The measured MTF cutoff frequency increased from 1.38 to $5.50 \mathrm{cy} / \mathrm{mm}$ for the last active cell (\#256) as the FOV decreased from 32 to $8 \mathrm{~cm}$; the corresponding modeled values were $1.39-5.55 \mathrm{cy} / \mathrm{mm}$.

The results of the measurement of the detector presampling MTF in the experimental VRX CT scanner are partly summarized in Fig. 6.9, where spatial frequencies were plotted as functions of the incident angle for several MTF values. The presented data were those acquired at the "typical" tube voltage of $80 \mathrm{kVp}$, because this tube voltage was the same at all the selected FOVs, allowing an MTF comparison in the entire range of the incident angles. The data clearly demonstrated the increase in the measured pre-reconstruction spatial resolution as the incident angle was decreasing, even with the high-resolution curves (for the MTF values of $0.1-0.3$ ) only partially measured at small incident angles due to the focal-spot limitation.

\subsection{Measurement of the scanner reconstruction MTF}

The reconstruction MTF, which described the post-reconstruction spatial resolution of the experimental VRX CT scanner, was measured by the LSF-phantom method, as explained in Section 5.6. Like before, the related LSF was examined first, before considering the measurement MTF results. A sample measured LSF that was used to compute the scanner reconstruction MTF is shown in Fig. 6.10. This LSF was acquired 


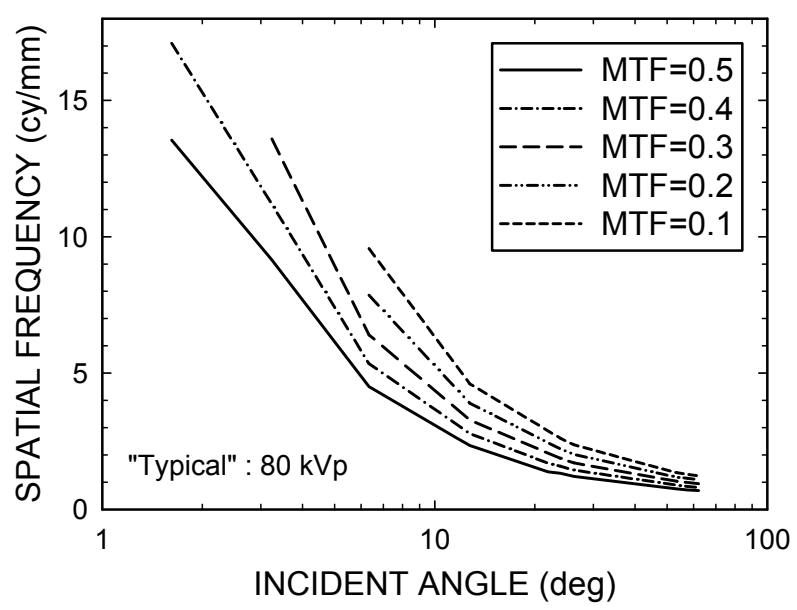

Figure 6.9. Measured spatial resolution of the VRX detector vs. incident angle.

Source: Reprinted with permission. R. Melnyk and F. A. DiBianca, "Modeling and measurement of the detector presampling MTF of a variable resolution x-ray CT scanner," Med. Phys. 34, 1062-1075, 2007. 


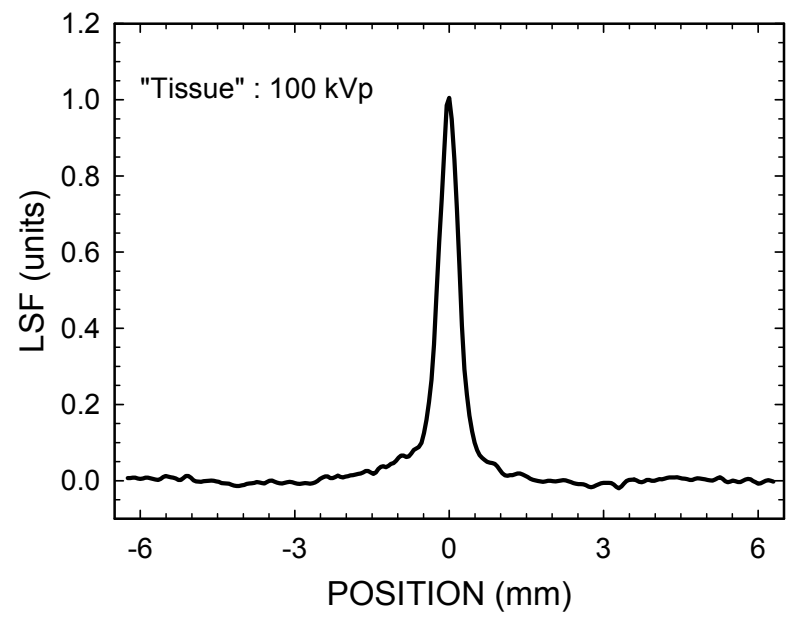

Figure 6.10. Sample measured LSF used to compute the measured scanner reconstruction MTF at 8-cm FOV. 
for the "tissue"-phantom tube voltage at 8-cm FOV. Contrary to the detector presampling LSF, the post-reconstruction LSF was rather symmetrical, with small differences between the tails attributed mainly to noise. The LSF symmetry was consistent with the properties of the resolution measures in conventional CT scanners. ${ }^{2,50}$ This symmetry also indicated that, despite the asymmetrical detector response contributing to each projection, the scanner response after reconstruction was symmetrical, as expected, because of the 360 $\operatorname{deg}$ CT scan.

The results of the measurement of the reconstruction MTF of the experimental VRX CT scanner by the LSF-phantom method are shown in Figs. 6.11-6.12 as solid lines with no symbols. These figures also include the measured detector presampling MTF for cell \#256 (dashed lines with symbols) as well as the ideal detector presampling MTF for the same cell (dotted lines with no symbols). The measured and ideal detector presampling MTFs were included only for reference and not for comparison with the scanner reconstruction MTF; these three functions (the former two and the latter) should not be directly compared nor expected to be similar as they represented different components of the scanner spatial resolution. This time, all the MTFs were given in the object plane (i.e., the plane that passed through the rotation axis of the rotary table and was orthogonal to the x-ray beam). Accordingly, the included detector presampling MTFs were projected from the detector plane, where they were originally determined, to the object plane.

As discussed in Section 5.6.2.4, the reconstruction MTF of the experimental VRX CT scanner was measured only at 8 and 16-cm FOVs, due to difficulties with, on one side, performing calibration at the smaller FOVs $(1-4 \mathrm{~cm})$ and, on the other side, 

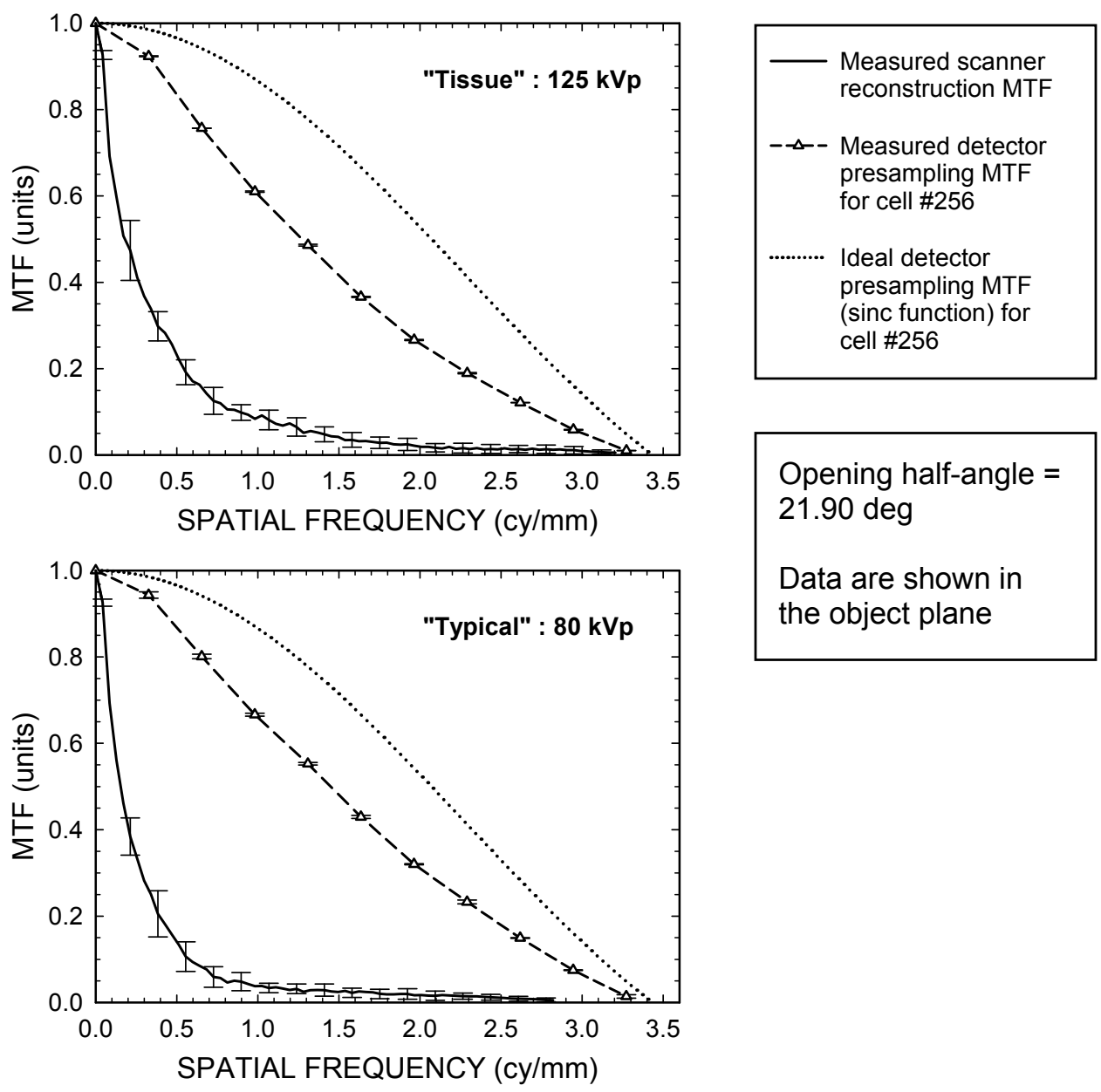

Opening half-angle $=$ $21.90 \mathrm{deg}$

Data are shown in the object plane

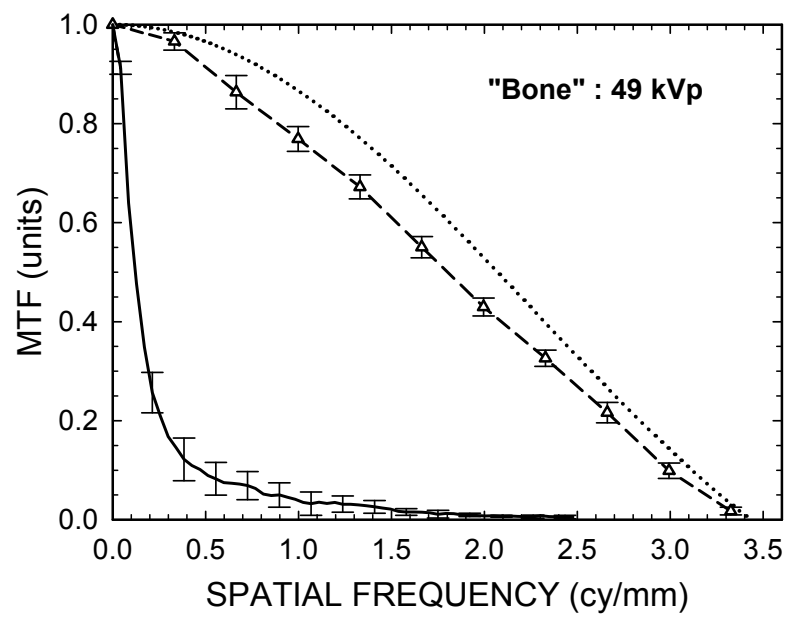

Figure 6.11. Measured scanner reconstruction MTF at 16-cm FOV. 

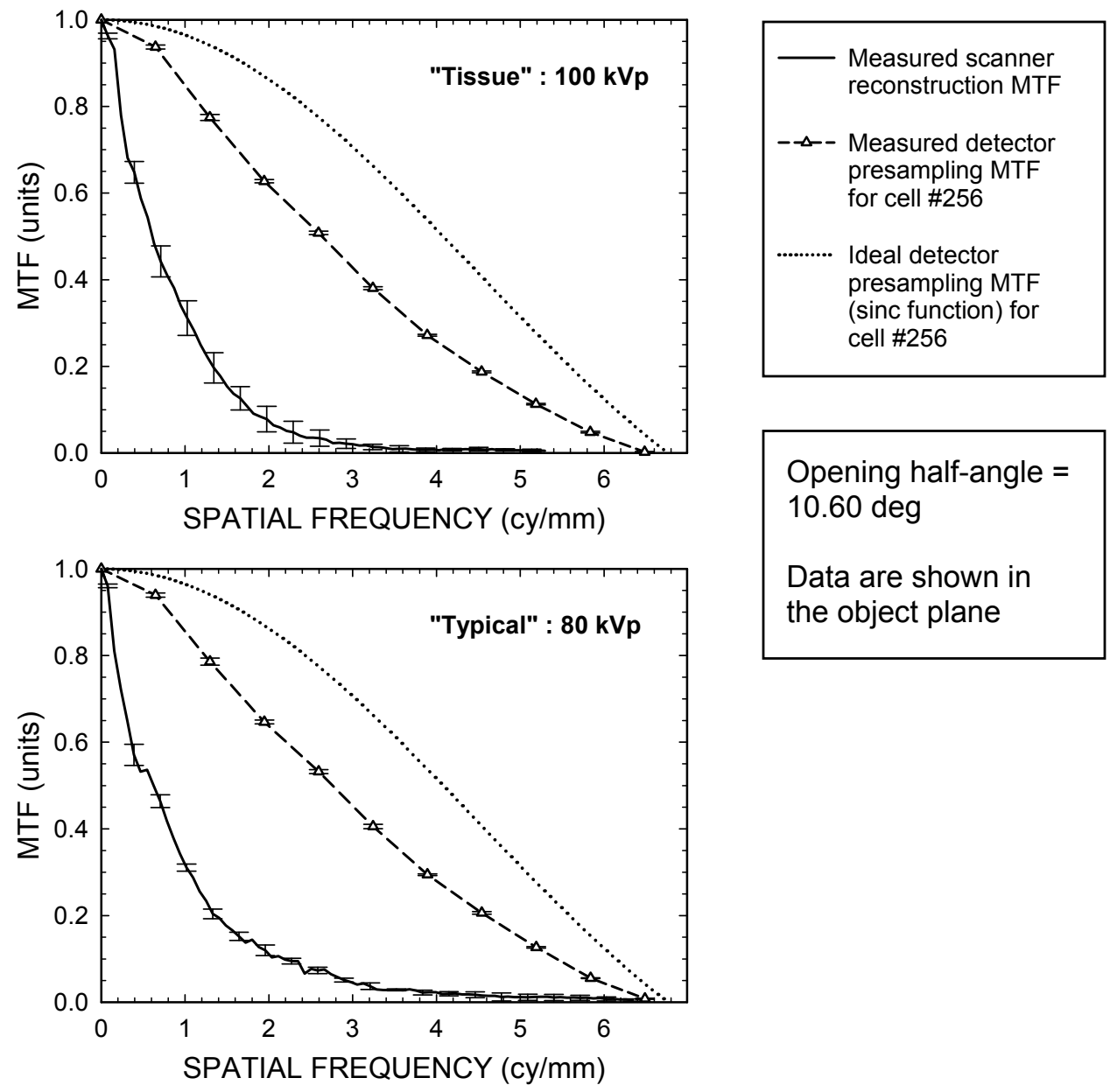

Opening half-angle $=$ $10.60 \mathrm{deg}$

Data are shown in the object plane

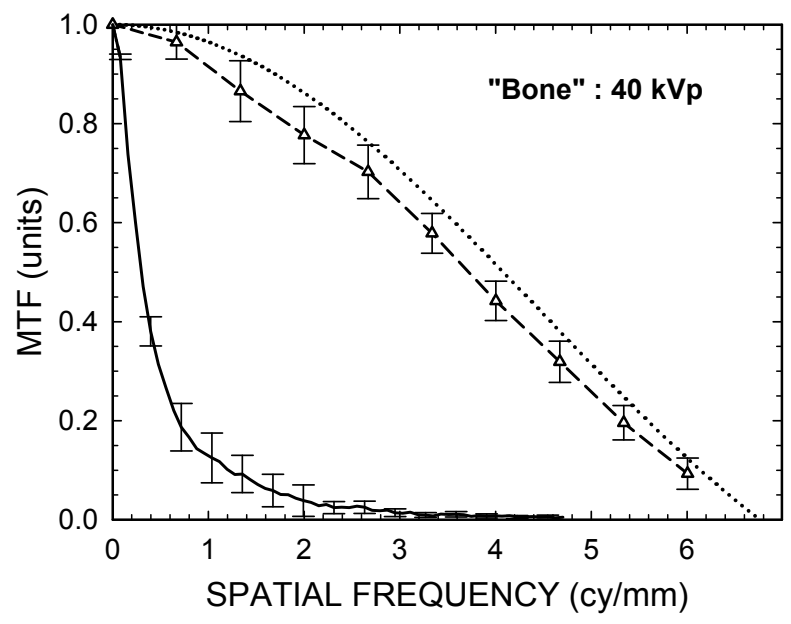

Figure 6.12. Measured scanner reconstruction MTF at 8-cm FOV. 
accurately reconstructing phantom images at the larger FOV $(32 \mathrm{~cm})$. Also, because the LSF phantom during the measurement was placed only in one position - i.e., in the middle of the top plate of the rotary table - the measured MTF represented the scanner spatial resolution only near the center of the reconstructed image.

As before, a few important observations were made from the results. First, the measured scanner reconstruction MTF curves and their corresponding cutoff frequencies were much lower than the measured detector presampling MTF curves with their cutoff frequencies. Although the reconstruction spatial resolution was expected to be lower than the detector resolution, this difference in the experimental VRX CT scanner seemed excessive compared with the typical difference found in CT. One possible explanation included the limiting effect of the focal spot. Thus, at 16-cm FOV, the cutoff frequency of the rectangular focal spot projected to the object plane was $2.27-4.92 \mathrm{cy} / \mathrm{mm}$ (for cells $\# 1-256)$ for the measured focal-spot size (1.23 $\mathrm{mm}$ at $80 \mathrm{kVp}$ and $1.50 \mathrm{~mm}$ at $40 \mathrm{kVp}$, as described in Section 5.5.2.3). At 8-cm FOV, the corresponding cutoff frequency was 2.27-5.34 cy/mm. Another explanation of the low measured values of the scanner reconstruction MTF referred to the fact that the calibration and, to a lesser extent, reconstruction algorithms were still in the development stages, not much optimized, and, therefore, not very accurate.

Second, unlike the modeled and measured detector presampling MTF data, the measured scanner reconstruction MTF curves were lowest at the lower ("bone"-phantom) tube voltage. At the higher ("typical" and "tissue"-phantom) tube voltages, a general trend was difficult to establish because the measurement results, available only at two FOVs, provided conflicting information. The low reconstruction MTF curves at the lower 
tube voltage - which was close or equal to $40 \mathrm{kVp}$ - could be explained by larger imagequality degradation caused by the increased size of the focal spot, due to the "blooming" effect. It was also interesting to note that the error bars at the lower tube voltage were not necessarily larger, despite the decreased SNR in the projection data.

Third, at each FOV, the reconstruction MTF cutoff frequencies for the three tube voltages were different. Assuming the cutoff frequency was defined via the 0.01-unit MTF threshold (as per description in Section 4.2.3), the values of this frequency at 16-cm FOV varied from the minimum of $1.84 \mathrm{cy} / \mathrm{mm}$ for the "bone"-phantom tube voltage to the maximum of $2.95 \mathrm{cy} / \mathrm{mm}$ for the "tissue"-phantom tube voltage; this corresponded to a $60 \%$ difference in the cutoff frequency. Under the same assumption at 8 -cm FOV, the minimum and maximum cutoff frequencies were $3.19 \mathrm{cy} / \mathrm{mm}$ for the "bone"-phantom tube voltage and $5.79 \mathrm{cy} / \mathrm{mm}$ for the "typical" tube voltage, respectively, amounting to an $82 \%$ difference. Such deviation of the reconstruction MTF cutoff frequency with the tube voltage was believed to result from calibration and reconstruction inaccuracies rather than from any physical phenomena. The lowest cutoff frequency at the "bone"-phantom tube voltage was due to, again, the increased focal-spot size at such a low voltage (49 and $40 \mathrm{kVp})$.

Fourth, despite the generally low measured reconstruction MTF of the experimental VRX CT scanner and only two FOVs at which this MTF was successfully evaluated, the increase in the scanner post-reconstruction spatial resolution with the decreasing FOV was evident. At the "bone"-phantom tube voltage, the reconstruction MTF cutoff frequency (defined, as previously, via the 0.01-unit MTF threshold) increased from 1.84 to $3.19 \mathrm{cy} / \mathrm{mm}$ as the FOV decreased from 16 to $8 \mathrm{~cm}$. At the 
"tissue"-phantom tube voltage, the same reduction in the FOV provided the cutofffrequency increase from 2.95 to $3.40 \mathrm{cy} / \mathrm{mm}$. When considering the average cutoff frequency among the three tube voltages, the equivalent increase was from 2.44 to $4.13 \mathrm{cy} / \mathrm{mm}$.

\subsection{Discussion of the results}

In the current study, the detector presampling MTF and the scanner reconstruction MTF, which represented the pre-reconstruction spatial resolution and the postreconstruction spatial resolution, respectively, of the experimental VRX CT scanner, were thoroughly evaluated. Based on the results of the evaluation, several important issues specific to this type of the scanner should be discussed.

First and foremost, an x-ray tube with a very small focal spot is required to achieve high spatial resolution of which the VRX detector is capable. As seen in the case of the pre-reconstruction spatial resolution, because of the relatively large focal spot used, the highest spatial frequency (in the detector plane) for which the detector presampling MTF could be measured was $20.90 \mathrm{cy} / \mathrm{mm}$ (for cell \#283 at 1-cm FOV). To be able to measure the detector presampling MTF up to the maximum frequency predicted by the Monte Carlo simulation (53.64 cy/mm for cell \#1 at 1-cm FOV), the focal spot with the actual size of no greater than $40 \mu \mathrm{m}$ (as explained later) is needed for the described measurement geometry. In the case of the post-reconstruction spatial resolution, the employed focal spot, along with the other contributing factors, limited the highest cutoff frequency (in the object plane) of the scanner reconstruction MTF to the average value of $4.13 \mathrm{cy} / \mathrm{mm}$ (at $8-\mathrm{cm}$ FOV). In order to obtain the scanner reconstruction MTF whose 
cutoff frequency is mainly determined by the maximum detector sampling and not by the focal spot - i.e., to take the full advantage of the increased detector resolution while operating in the CT mode - the actual focal-spot size not exceeding $82 \mu \mathrm{m}$ is required. Thus, with the sufficiently-small focal spot, the VRX CT scanner, in the presented configuration, is expected to provide the pre-reconstruction spatial resolution of more than $50 \mathrm{cy} / \mathrm{mm}$ and the post-reconstruction spatial resolution that reflects this high detector resolution and is not limited by the focal-spot size. A more detailed analysis of the desired x-ray tube parameters will be given in the later section.

Next, the system magnification in the VRX CT scanner varies more from the detector centerline to the periphery than in conventional CT scanners. For the measurement of the detector presampling MTF, the system magnification changed from 1.035 for cell \#283 at 2-cm FOV to 1.350 for cell $\# 1$ at 1 -cm FOV. These values were slightly larger when measuring the scanner reconstruction MTF - i.e., in the actual CT mode - because, due to the location of the rotary table, an LSF phantom was positioned farther from the VRX detector compared with the slit used for the detector presampling MTF measurement. Specifically, in the case of the scanner reconstruction MTF, the system magnification changed from 1.174 for cell \#256 at 1-cm FOV to 1.415 for cell \#1 at all the FOVs. Such variation in the system magnification has a great impact on the scanner spatial resolution, ultimately requiring a smaller focal spot for high-resolution CT imaging.

Additionally, the beam quality (determined primarily by the x-ray tube voltage) directly affects the pre-reconstruction spatial resolution of the VRX CT scanner but appears to have no consistent influence on the scanner post-reconstruction spatial 
resolution. As shown by the detector presampling MTF results, higher beam quality lowered the detector resolution. The reason for this was twofold. First, higher x-ray energy increased the depth of x-ray penetration, which, because of the inherent detector angulation, enlarged the lateral component of the x-ray penetration length. Second, higher x-ray energy increased the size of the deposited energy clouds. It should be noted that the second phenomenon occurs in all x-ray detectors. In terms of the scanner reconstruction MTF, the absence of any consistent dependence of the results on the beam quality was probably due to the limited accuracy of the scanner calibration and the subsequent image reconstruction.

The obtained results of the comprehensive evaluation of the pre-reconstruction and post-reconstruction spatial resolution of the experimental VRX CT scanner are of great importance. For the detector presampling MTF, the theoretical and experimental results showed that the measured cutoff frequencies agreed well with the modeling values, and both the measured and modeled cutoff frequencies were close to the ideal values. The detector presampling MTF results also demonstrated reasonably good agreement between the measured and the modeled MTF curves. The maximum deviation between the measured and the modeled MTFs ( $\left.\left|\mathrm{MTF}_{\text {meas }}-\mathrm{MTF}_{\text {mod }}\right|\right)$ was 0.23 units, and the average deviation, excluding the normalized zero-frequency points, was 0.05 units. In addition, the collected data indicated that the detector presampling LSF asymmetry due to cell-to-cell x-ray penetration only slightly reduced the corresponding MTF but did not significantly affect the cutoff frequency. Furthermore, the detector presampling MTF results underscored the necessity of using a very small focal spot for achieving high prereconstruction spatial resolution in the VRX CT scanner. 
For the scanner reconstruction MTF, on the other hand, the experimental results revealed that the employed calibration and reconstruction procedures could not often provide sufficient accuracy. The largest error was believed to be introduced by the scanner calibration, as this procedure was built on several idealizations (perfect detector geometry, ideal vertical alignment of the scanner components, etc.) and was also very sensitive to fluctuations in the "constant" parameters (the angular speed of the rotary table, the distance from the pin to the rotation axis, and the distance from the focal spot to the rotation axis). The limited accuracy of the calibration and reconstruction procedures was also due to the fact that these techniques were still being developed and were not fully optimized. Thus, the scanner reconstruction MTF results emphasized the importance of an accurate and reliable calibration and reconstruction in the VRX CT scanner. Additionally, these results supported the previous finding regarding the need for a very small focal spot, in order to achieve high post-reconstruction spatial resolution.

Despite the discussed limitations, the obtained results for both the detector presampling MTF and the scanner reconstruction MTF clearly showed the increase in spatial resolution of the VRX CT scanner with the decreasing FOV. Hence, the current study confirmed the potential value of the VRX detection technique for high-resolution CT imaging.

\subsection{Preferred x-ray tube parameters}

Because the x-ray tube used in the current study limited the measurement of both the pre-reconstruction and the post-reconstruction spatial resolution in the experimental VRX CT scanner, it is of practical interest to find the x-ray tube parameters that would 
permit such measurement up to the highest spatial frequencies. A main set of parameters includes the focal-spot width and length, the target angle of the anode, the anode heatstorage capacity and cooling rate, the housing heat-storage capacity and cooling rate, the tube output (photon fluence or x-ray exposure per tube current and time), and the maximum tube current allowable at a given tube voltage over a specified period of time. ${ }^{2}$ Among these parameters, only the width and length of the focal spot can be easily determined, based on the spatial-resolution requirements. The accurate values of the other parameters, which mostly depend on the thermal limitations of the tube, can only be found via complex computations and sophisticated modeling. ${ }^{87}$ However, it is possible to obtain a simple performance estimate for a desired $\mathrm{x}$-ray tube by assuming that its design is very similar to the tube that was employed in the study.

The case of the pre-reconstruction spatial resolution will be considered first. If one uses the earlier criterion (Section 6.2), according to which the detector presampling MTF curves were terminated at the spatial frequency not exceeding $75 \%$ of the focal-spot cutoff frequency (to avoid degradation of the MTF curves by the focal spot), the following relationship can be established between the cutoff frequency $f_{\mathrm{DET}}$ of the detector presampling MTF and the cutoff frequency $f_{\mathrm{FS}}^{*}$ of the focal-spot MTF as projected to the detector plane:

$$
f_{\mathrm{FS}}^{*} \geq 1.33 f_{\mathrm{DET}} \text {. }
$$

Assuming that the focal-spot cutoff frequency is reciprocal to the focal-spot width and expressing the projected focal spot (in the detector plane) via the actual focal spot (in the focal-spot plane) and the system magnification $m$, one gets 


$$
w_{\mathrm{FS}} \leq \frac{1}{1.33(m-1) f_{\mathrm{DET}}},
$$

where $w_{\mathrm{FS}}$ is the actual focal-spot width. For the maximum modeled cutoff frequency of the detector presampling MTF (53.64 cy/mm for cell $\# 1$ at $1-\mathrm{cm} \mathrm{FOV)} \mathrm{and} \mathrm{the}$ corresponding system magnification (1.350), the right side of Eq. 6.2 is equal to $40 \mu \mathrm{m}$. Thus, the largest width of the actual focal spot that would allow the detector presampling MTF measurement up to the maximum modeled cutoff frequency is $40 \mu \mathrm{m}$ for the described measurement geometry. Choosing this value as the preferred focal-spot width and assuming that the focal spot is twice this size in the other direction, one obtains the actual focal-spot dimensions of 40 by $80 \mu \mathrm{m}$ for the desired $\mathrm{x}$-ray tube.

Although, as mentioned before, the calculation of the thermal properties of an $\mathrm{x}$ ray tube is rather complex, new-tube performance can be readily estimated by scaling from the design of the x-ray tube with known performance, i.e., the tube used in the current study. The basis for such scaling is given by the expression that relates the power $P$ of a rotating-anode tube and the temperature rise $\Delta T_{\mathrm{FS}}$ in the area of the focal spot for very short exposure times: ${ }^{87}$

$$
P=k_{1} L \sqrt{w_{\mathrm{FS}} D n} \Delta T_{\mathrm{FS}},
$$

where $k_{1}$ is a constant, $L$ is the width of the focal track, $D$ is the mean diameter of the focal track, and $n$ is the rotational speed (revolutions/s) of the anode. For short exposures that start from the same thermal conditions on the track, $\Delta T_{\mathrm{FS}}$ can be considered constant. ${ }^{87}$ Then, utilizing the relationship between the focal-track width $L$ and the focalspot length $h_{F S}$, Eq. 6.3 can be rewritten as 


$$
P=k_{2} \frac{h_{\mathrm{FS}} \sqrt{w_{\mathrm{FS}} D n}}{\sin \beta},
$$

where $k_{2}$ is another constant, and $\beta$ is the target angle of the anode.

Using Eq. 6.4, one can find the power $P$ of the desired x-ray tube in terms of the power $P_{0}$ of the employed tube. Assuming that the only difference between the two tubes is the focal-spot size, it can be shown that

$$
P=\frac{h_{\mathrm{FS}}}{h_{\mathrm{FS} 0}} \sqrt{\frac{w_{\mathrm{FS}}}{w_{\mathrm{FS} 0}}} P_{0},
$$

where $h_{\mathrm{FS} 0}$ and $w_{\mathrm{FS} 0}$ are the focal-spot length and width, respectively, of the employed tube. The x-ray tube utilized in the current study had the $0.6-\mathrm{mm}$ nominal focal spot. Hence, the actual focal-spot width and length were in the range of 0.6-0.9 $\mathrm{mm}$ and 0.9$1.3 \mathrm{~mm}$, respectively. ${ }^{90}$ The measured focal-spot length was $1.23 \mathrm{~mm}$ (Section 5.5.2.3). By selecting the values of 0.8 and $1.2 \mathrm{~mm}$ for the focal-spot width and length, respectively, of the employed tube and using the previously-determined values of 40 and $80 \mu \mathrm{m}$ for the same parameters of the desired tube, one obtains

$$
P=0.0149 P_{0}
$$

which means that, based on the permissible temperature rise in the focal-spot area, the desired tube would have 67 times less power than the tube utilized in the study. Because the computed power is lower, the other temperature rises (for the focal track, anode, bearing assembly, etc.) should not impose any further limitations and can be ignored. As a result of the reduced power, one would have to increase the sampling time by the factor of 67 when using the desired tube, in order to get the same SNR; the new sampling time would be approximately $170 \mathrm{~ms}$. 
In the case of the post-reconstruction spatial resolution, the system magnification is slightly larger (1.415 for cell \#1), and if the maximum cutoff frequency of the modeled detector presampling MTF is used again as a criterion, the actual focal-spot width given by Eq. 6.2 would be even smaller, about $34 \mu \mathrm{m}$. However, the scanner reconstruction MTF is often limited by the Nyquist frequency and not by the cutoff frequency of the detector presampling MTF. The maximum Nyquist frequency for the described geometry was $22.09 \mathrm{cy} / \mathrm{mm}$ (for cell \#1 at 1-cm FOV). For this frequency, Eq. 6.2 gives the largest width of the actual focal spot equal to $82 \mu \mathrm{m}$. Clearly, the previously-discussed x-ray tube, which would allow accurate measurement of the detector presampling MTF, would be more than adequate for high-quality measurement of the scanner reconstruction MTF.

In general, if one wishes to consider the preferred $\mathrm{x}$-ray tube parameters for operating a practical VRX CT scanner, a much more sophisticated analysis would be required, as was stated at the beginning of this section. As a minimum, an x-ray tube with multiple focal spots would be needed. It could also be necessary to employ even two separate $\mathrm{x}$-ray tubes - a high-power, short-exposure, rotating-anode tube and a lowpower, long-exposure, stationary-anode tube. 


\section{CHAPTER 7. CONCLUSIONS*}

A comprehensive evaluation of spatial resolution of the experimental VRX CT scanner was done for the first time and is presented in this dissertation. Two components of the scanner spatial resolution were evaluated - the pre-reconstruction spatial resolution and the post-reconstruction spatial resolution. In terms of the common resolution measures, the pre-reconstruction spatial resolution was described by the detector presampling MTF, whereas the post-reconstruction spatial resolution was given by the scanner reconstruction MTF.

The detector presampling MTF of the experimental VRX CT scanner was evaluated both theoretically (by the Monte Carlo simulation) and experimentally (by the moving-slit method). The theoretical results showed the increase in the maximum cutoff frequency of the detector presampling MTF (in the detector plane) from 1.53 to $53.64 \mathrm{cy} / \mathrm{mm}$ as the FOV of the scanner decreased from 32 to $1 \mathrm{~cm}$. The experimental cutoff frequencies agreed well with the theoretical values in all the cases where such comparison could be made. The agreement between the experimental and the theoretical MTF curves, however, was not as good, but the discrepancies could be explained by the physical phenomena present in the actual scanner but not included in the Monte Carlo simulation. The detector presampling MTF could not be measured for the highmagnification cells at the small FOVs $(1-8 \mathrm{~cm})$ because of the relatively large focal-spot size of the employed x-ray tube. But the measurements that could be made supported the

\footnotetext{
* Adapted with permission. R. Melnyk and F. A. DiBianca, "Modeling and measurement of the detector presampling MTF of a variable resolution x-ray CT scanner," Med. Phys. 34, 1062-1075, 2007.
} 
validity of the MTF modeling by the Monte Carlo simulation.

The reconstruction MTF of the experimental VRX CT scanner was evaluated only experimentally (by the LSF-phantom method). Unfortunately, the evaluation could only be done at 8 and 16-cm FOVs, due to limitations of the calibration and reconstruction algorithms available at the time of the study. Also, because of the relatively large focalspot size and the calibration-reconstruction inaccuracies, the measured scanner reconstruction MTF curves and their cutoff frequencies were considerably lower than the corresponding detector presampling MTF curves with their cutoff frequencies. Despite the limited number of measurements and the lower than expected MTF results, the experimental data demonstrated the increase in the average cutoff frequency of the scanner reconstruction MTF (in the object plane) from 2.44 to $4.13 \mathrm{cy} / \mathrm{mm}$ as the FOV of the scanner decreased from 16 to $8 \mathrm{~cm}$.

The described comprehensive evaluation of the pre-reconstruction and postreconstruction spatial resolution of the experimental VRX CT scanner is an important step in the assessment of the scanner's performance. The results of the study can be used for evaluating other image-quality parameters as well as for optimizing the scanner design to maximize its spatial resolution. The study also emphasizes the importance of employing a small-focal-spot $\mathrm{x}$-ray tube as well as improving the accuracy and reliability of the calibration and reconstruction procedures, in order to achieve in practice the spatial resolution predicted by the modeling. Overall, the study supports the great potential of the VRX CT scanner for improving and varying spatial resolution in biological x-ray imaging. 


\section{LIST OF REFERENCES}

1. M. P. Siedband, "Medical imaging systems," in Medical Instrumentation. Application and Design, edited by J. G. Webster, John Wiley \& Sons, New York, 518-576, 1998.

2. J. Hsieh, Computed Tomography. Principles, Design, Artifacts, and Recent Advances, SPIE Press, Bellingham, 2003.

3. J. Bull, "History of computed tomography," in Radiology of the Skull and Brain. Volume 5. Technical Aspects of Computed Tomography, edited by T. H. Newton and D. G. Potts, C. V. Mosby Company, St. Louis, 3835-3849, 1981.

4. L. M. Zatz, "Basic principles of computed tomography scanning," in Radiology of the Skull and Brain. Volume 5. Technical Aspects of Computed Tomography, edited by T. H. Newton and D. G. Potts, C. V. Mosby Company, St. Louis, 3853-3876, 1981.

5. Mathematics and Physics of Emerging Biomedical Imaging, edited by Committee on the Mathematics and Physics of Emerging Dynamic Biomedical Imaging, National Academy Press, Washington, 1996.

6. D. J. Goodenough, "Tomographic imaging," in Handbook of Medical Imaging. Volume 1. Physics and Psychophysics, edited by J. Beutel, H. L. Kundel, and R. L. Van Metter, SPIE Press, Bellingham, 511-554, 2000.

7. H. H. Barrett and W. Swindell, Radiological Imaging. The Theory of Image Formation, Detection, and Processing, Academic Press, San Diego, 2001.

8. A. Webb, Introduction to Biomedical Imaging, IEEE Press, Piscataway, 2003.

9. J. T. Bushberg, J. A. Seibert, E. M. Leidholdt, and J. M. Boone, The Essential Physics of Medical Imaging, Williams \& Wilkins, Baltimore, 1994.

10. A. Macovski, Medical Imaging Systems, Prentice Hall, Englewood Cliffs, 1983.

11. W. A. Kalender, "X-ray computed tomography," Phys. Med. Biol. 51, R29-R43, 2006.

12. E. Hayes, "Variety, ingenuity spice up latest CT scanner designs," Diagn. Imaging 11, 124-128, 2006.

13. S. Achenbach, D. Ropers, A. Kuettner, T. Flohr, B. Ohnesorge, H. Bruder, H. Theessen, M. Karakaya, W. G. Daniel, W. Bautz, W. A. Kalender, and K. Anders, "Contrast-enhanced coronary artery visualization by dual-source computed tomography - initial experience," Eur. J. Radiol. 57, 331-335, 2006. 
14. E. L. Ritman, "Micro-computed tomography - current status and developments," Ann. Rev. Biomed. Eng. 6, 185-208, 2004.

15. M. J. Paulus, S. S. Gleason, S. J. Kennel, P. R. Hunsicker, and D. K. Johnson, "High resolution x-ray computed tomography: an emerging tool for small animal cancer research," Neoplasia 2, 62-70, 2000.

16. D. W. Holdsworth and M. M. Thornton, "Micro-CT in small animal and specimen imaging," Trends Biotechnol. 20, S34-S39, 2002.

17. S. C. Lee, H. K. Kim, I. K. Chun, M. H. Cho, S. Y. Lee, and M. H. Cho, "A flat-panel detector based micro-CT system: performance evaluation for small-animal imaging," Phys. Med. Biol. 48, 4173-4185, 2003.

18. S. Greschus, F. Kiessling, M. P. Lichy, J. Moll, M. M. Muelier, R. Savai, F. Rose, C. Ruppert, A. Günther, M. Luecke, N. E. Fusenig, W. Semmler, and H. Traupe, "Potential applications of flat-panel volumetric CT in morphologic and functional small animal imaging," Neoplasia 7, 730-740, 2005.

19. G. Wang, S. Zhao, H. Yu, C. A. Miller, P. J. Abbas, B. J. Gantz, S. W. Lee, and J. T. Rubinstein, "Design, analysis and simulation for development of the first clinical micro-CT scanner," Acad. Radiol. 12, 511-525, 2005.

20. S. Robinson, A. Suomalainen, and M. Kortesniemi, “ $\mu$-CT,” Eur. J. Radiol. 56, 185191, 2005.

21. A. Sasov, "Comparison of fan-beam, cone-beam and spiral scan reconstruction in xray micro-CT," Proc. SPIE 4503, 124-131, 2002.

22. L. A. Feldkamp, L. C. Davis, and J. W. Kress, "Practical cone-beam algorithm," J. Opt. Soc. Am. A 1, 612-619, 1984.

23. A. Sasov and D. Dewaele, "High-resolution in-vivo micro-CT scanner for small animals," Proc. SPIE 4503, 256-264, 2002.

24. A. Sasov, "Desktop x-ray micro-CT instruments," Proc. SPIE 4503, 282-290, 2002.

25. W. S. Haddad, I. McNulty, J. E. Trebes, E. H. Anderson, R. A. Levesque, and L. Yang, "Ultrahigh-resolution x-ray tomography," Science 266, 1213-1215, 1994.

26. B. P. Flannery, H. W. Deckman, W. G. Roberge, and K. L. D'Amico, "Threedimensional x-ray microtomography," Science 237, 1439-1444, 1987.

27. E. L. Ritman, "Molecular imaging in small animals - roles for micro-CT," J. Cell. Biochem. Suppl. 39, 116-124, 2002. 
28. N. L. Ford, M. M. Thornton, and D. W. Holdsworth, "Fundamental image quality limits for microcomputed tomography in small animals," Med. Phys. 30, 2869-2877, 2003.

29. C. Badea, L. W. Hedlund, and G. A. Johnson, "Micro-CT with respiratory and cardiac gating," Med. Phys. 31, 3324-3329, 2004.

30. C. T. Badea, B. Fubara, L. W. Hedlund, and G. A. Johnson, "4-D micro-CT of the mouse heart," Mol. Imaging 4, 110-116, 2005.

31. H. L. Fred, "Drawbacks and limitations of computed tomography," Texas Heart Institute J. 31, 345-348, 2004.

32. F. A. DiBianca, V. Gupta, and H. D. Zeman, "A variable resolution x-ray detector for computed tomography: I. Theoretical basis and experimental verification," Med. Phys. 27, 1865-1874, 2000.

33. V. Gupta, Variable Resolution X-Ray Imaging System, MS Thesis, University of Tennessee, Memphis, 1998.

34. F. A. DiBianca, V. Gupta, P. Zou, L. M. Jordan, J. S. Laughter, H. D. Zeman, and J. I. Sebes, "Ultrahigh-resolution CT and DR scanner," Proc. SPIE 3659, 56-64, 1999.

35. F. A. DiBianca, P. Zou, L. M. Jordan, J. S. Laughter, and H. D. Zeman, “A variable resolution $\mathrm{X}$-ray detector for computed tomography: II. Imaging theory and performance," Med. Phys. 27, 1875-1880, 2000.

36. R. Melnyk and F. A. DiBianca, "Monte Carlo study of x-ray cross-talk in a variable resolution x-ray detector," Proc. SPIE 5030, 694-701, 2003.

37. F. A. DiBianca, R. Melnyk, C. Duckworth, S. Russ, L. M. Jordan, and J. S. Laughter, “Comparison of VRX CT scanner geometries," Proc. SPIE 4320, 627-635, 2001.

38. D. A. Rendon, F. A. DiBianca, and G. S. Keyes, "Comparison of multi-arm VRX CT scanners through computer models," Proc. SPIE 6510, 65103Y1-65103Y9, 2007.

39. F. A. DiBianca, D. Gulabani, L. M. Jordan, S. Vangala, D. Rendon, J. S. Laughter, R. Melnyk, M. W. Gaber, and G. S. Keyes, "Four-arm variable-resolution x-ray detector for CT target imaging," Proc. SPIE 5745, 332-339, 2005.

40. F. A. DiBianca, R. Melnyk, A. Sambari, L. M. Jordan, J. S. Laughter, and P. Zou, "A solid-state VRX CT detector," Proc. SPIE 3977, 205-210, 2000.

41. L. M. Jordan, F. A. DiBianca, P. Zou, J. Laughter, and H. Zeman, "Processing of CT sinograms acquired using a VRX detector," Proc. SPIE 3977, 570-579, 2000. 
42. L. M. Jordan, F. A. DiBianca, R. Melnyk, A. Choudhary, H. Shukla, J. Laughter, and M. W. Gaber, "Determination of calibration parameters of a VRX CT system using an 'Amoeba' algorithm,” J. X-ray Science Technol. 12, 281-293, 2004.

43. B. Dahi, G. S. Keyes, D. A. Rendon, and F. A. DiBianca, "Performance analysis of a CsI-based flat panel detector in a cone beam variable resolution $\mathrm{x}$-ray system," Proc. SPIE 6510, 65104B1-65104B8, 2007.

44. R. Melnyk and F. A. DiBianca, "Modeling and measurement of the detector presampling MTF of a variable resolution X-ray CT scanner," Med. Phys. 34, 10621075, 2007.

45. J. T. Dobbins III, "Image quality metrics for digital systems," in Handbook of Medical Imaging. Volume 1. Physics and Psychophysics, edited by J. Beutel, H. L. Kundel, and R. L. Van Metter, SPIE Press, Bellingham, 161-222, 2000.

46. J. L. Lancaster, Physics of Medical X-Ray Imaging, http://ric.uthscsa.edu/ personalpages/lancaste/DI II.html, accessed March 30, 2007.

47. K. Rossmann, "Point spread-function, line spread-function, and modulation transfer function. Tools for the study of imaging systems," Radiology 93, 257-272, 1969.

48. C. E. Metz and K. Doi, "Transfer function analysis of radiographic imaging systems," Phys. Med. Biol. 24, 1079-1106, 1979.

49. I. A. Cunningham, "Applied linear-systems theory," in Handbook of Medical Imaging. Volume 1. Physics and Psychophysics, edited by J. Beutel, H. L. Kundel, and R. L. Van Metter, SPIE Press, Bellingham, 79-159, 2000.

50. S. M. Blumenfeld and G. Glover, "Spatial resolution in computed tomography," in Radiology of the Skull and Brain. Volume 5. Technical Aspects of Computed Tomography, edited by T. H. Newton and D. G. Potts, C. V. Mosby Company, St. Louis, 3918-3940, 1981.

51. R. H. Vollmerhausen and R. G. Driggers, Analysis of Sampled Imaging Systems, SPIE Press, Bellingham, 2000.

52. H. H. Barrett and K. J. Myers, Foundations of Image Science, Wiley \& Sons, Hoboken, 2004.

53. G. D. Boreman, Modulation Transfer Function in Optical and Electro-Optical Systems, SPIE Press, Bellingham, 2001.

54. J. E. Gray and M. Trefler, "Phase effects in diagnostic radiological images," Med. Phys. 3, 195-203, 1976. 
55. M. L. Giger and K. Doi, "Investigation of basic imaging properties in digital radiography. I. Modulation transfer function," Med. Phys. 11, 287-295, 1984.

56. H. Fujita, K. Doi, and M. L. Giger, "Investigation of basic imaging properties in digital radiography. 6. MTFs of II-TV digital imaging systems," Med. Phys. 12, 713$720,1985$.

57. J. T. Dobbins III, "Effects of undersampling on the proper interpretation of modulation transfer function, noise power spectra, and noise equivalent quanta of digital imaging systems," Med. Phys. 22, 171-181, 1995.

58. M. Lundqvist, Silicon Strip Detectors for Scanned Multi-Slit X-Ray Imaging, http://www.particle.kth.se/htmlnew/publications/files/mats.pdf, accessed March 19, 2007.

59. M. Albert and A. D. A. Maidment, "Linear response theory for detectors consisting of discrete arrays," Med. Phys. 27, 2417-2434, 2000.

60. I. A. Cunningham and B. K. Reid, "Signal and noise in modulation transfer function determinations using the slit, wire, and edge techniques," Med. Phys. 19, 1037-1044, 1992.

61. S. Nagarajan, Experimental Study and Optimization of the Spatial Resolution of a Kinestatic Charge Detector for Digital Radiography, MS Thesis, University of Tennessee, Memphis, 1995.

62. J. Morishita, K. Doi, R. Bollen, P. C. Bunch, D. Hoeschen, G. Sirand-rey, and Y. Sukenobu, "Comparison of two methods for accurate measurement of modulation transfer functions of screen-film systems," Med. Phys. 22, 193-200, 1995.

63. K. Doi, K. Strubler, and K. Rossmann, "Truncation errors in calculating the MTF of radiographic screen-film systems from the line spread function," Phys. Med. Biol. 17, 241-250, 1972.

64. C. E. Metz, K. A. Strubler, and K. Rossmann, "Choice of line spread function sampling distance for computing the MTF of radiographic screen-film systems," Phys. Med. Biol. 17, 638-647, 1972.

65. I. A. Cunningham and A. Fenster, "A method for modulation transfer function determination from edge profiles with correction for finite-element differentiation," Med. Phys. 14, 533-537, 1987.

66. A. Gopal and S. S. Samant, "Bar-pattern technique for modulation transfer function measurement in portal imaging," Proc. SPIE 5030, 433-444, 2003. 
67. H. Fujita, D. Y. Tsai, T. Itoh, K. Doi, J. Morishita, K. Ueda, and A. Ohtsuka, “A simple method for determining the modulation transfer function in digital radiography," IEEE Trans. Med. Imaging 11, 34-39, 1992.

68. J. T. Dobbins III, D. L. Ergun, L. Rutz, D. A. Hinshaw, H. Blume, and D. C. Clark, "DQE $(f)$ of four generations of computed radiography acquisition devices," Med. Phys. 22, 1581-1593, 1995.

69. C. D. Bradford, W. W. Peppler, and J. T. Dobbins III, "Performance characteristics of a Kodak computed radiography system," Med. Phys. 26, 27-37, 1999.

70. E. Samei, N. T. Ranger, J. T. Dobbins III, and Y. Chen, "Intercomparison of methods for image quality characterization. I. Modulation transfer function," Med. Phys. 33, 1454-1465, 2006.

71. E. Samei, M. J. Flynn, and D. A. Reimann, "A method for measuring the presampled MTF of digital radiographic systems using an edge test device," Med. Phys. 25, 102113, 1998.

72. P. B. Greer and T. Van Doorn, "Evaluation of an algorithm for the assessment of the MTF using an edge method," Med. Phys. 27, 2048-2059, 2000.

73. E. Buhr, S. Günther-Kohfahl, and U. Neitzel, “Accuracy of a simple method for deriving the presampled modulation transfer function of a digital radiographic system from an edge image," Med. Phys. 30, 2323-2331, 2003.

74. M. Bergstrom, "Performance evaluation of scanners," in Radiology of the Skull and Brain. Volume 5. Technical Aspects of Computed Tomography, edited by

T. H. Newton and D. G. Potts, C. V. Mosby Company, St. Louis, 4212-4227, 1981.

75. E. L. Nickoloff and R. Riley, "A simplified approach for modulation transfer function determinations in computed tomography," Med. Phys. 12, 437-442, 1985.

76. J. M. Boone, "Determination of the presampled MTF in computed tomography," Med. Phys. 28, 356-360, 2001.

77. Specification and Acceptance Testing of Computed Tomography Scanners. AAPM Report No. 39, American Association of Physicists in Medicine, Chicago, 1993.

78. M. Ohkubo, S. Wada, T. Matsumoto, and K. Nishizawa, "An effective method to verify line and point spread functions measured in computed tomography," Med. Phys. 33, 2757-2764, 2006.

79. P. F. Judy, "The line spread function and modulation transfer function of a computed tomographic scanner," Med. Phys. 3, 233-236, 1976. 
80. R. T. Droege and R. L. Morin, "A practical method to measure the MTF of CT scanners," Med. Phys. 9, 758-760, 1982.

81. P. C. Johns and M. J. Yaffe, "Theoretical optimization of dual-energy x-ray imaging with application to mammography," Med. Phys. 12, 289-296, 1985.

82. NIST XCOM: Photon Cross Sections Database, National Institute of Standards and Technology, http://physics.nist.gov/PhysRefData/Xcom/Text/XCOM.html, accessed February 24, 2004.

83. Varian X-ray Products: G1582TRI/G1582BI/G1582BI-G Rotating Anode X-ray Tube. Product Specifications, Varian Medical Systems, Salt Lake City, 2004.

84. J. A. Halbleib, R. P. Kensek, T. A. Mehlhorn, G. D. Valdez, S. M. Seltzer, and M. J. Berger, ITS Version 3.0: the Integrated TIGER Series of Coupled Electron/Photon Monte Carlo Transport Codes. Report SAND91-1634, Sandia National Laboratories, Albuquerque, 1992.

85. R. Birch and M. Marshall, "Computation of bremsstrahlung x-ray spectra and comparison with spectra measured with a Ge(Li) detector," Phys. Med. Biol. 24, 505$517,1979$.

86. M. R. Ay, S. Sarkar, M. Shahriari, D. Sardari, and H. Zaidi, “Assessment of different computational models for generation of $\mathrm{x}$-ray spectra in diagnostic radiology and mammography," Med. Phys. 32, 1660-1675, 2005.

87. J. A. Randmer, T. J. Koller, and W. P. Holland, "X-ray sources and controls," in Radiology of the Skull and Brain. Volume 5. Technical Aspects of Computed Tomography, edited by T. H. Newton and D. G. Potts, C. V. Mosby Company, St. Louis, 4058-4095, 1981.

88. W. H. Press, B. P. Flannery, S. A. Teukolsky, and W. T. Vetterling, Numerical Recipes. The Art of Scientific Computing, Cambridge University Press, Cambridge, 1987.

89. G. T. Herman, "Advanced principles of reconstruction algorithms," in Radiology of the Skull and Brain. Volume 5. Technical Aspects of Computed Tomography, edited by T. H. Newton and D. G. Potts, C. V. Mosby Company, St. Louis, 3888-3903, 1981.

90. P. Rastello, X-ray Tube Training. Technology and Parameters, http://www.e -radiography.net/radtech/x/xray tubes.pdf, accessed September 23, 2004. 


\section{VITA}

Roman Melnyk was born in Ternopil, Ukraine in 1973. He attended Ternopil State Technical University from 1991 till 1996 and graduated with an Engineering degree in Biomedical Engineering. After graduation, he worked as a Research Engineer in the Department of Biomedical Systems and Apparatuses at Ternopil State Technical University. He entered the University of Tennessee Health Science Center in 1999 and graduated with a Master of Science degree in Biomedical Engineering in 2001. He continued his study at the University of Tennessee Health Science Center from 2002, pursuing a Doctor of Philosophy degree in Biomedical Engineering and Imaging. 